\title{
Sur les extensions des groupes topologiques.
}

\author{
Mémoire de Lorenzo Calabi (à Strasbourg).
}

Résame. - Etude du problème suivant: deux groupes topologiques $\mathbf{B}$ et $\mathbf{F}$ étant donnés, $y$ a-t-il des groupes $\mathrm{E}$ admettant $\mathrm{F}$ comme sous-groupe fermé distingué, tels que ElF soit homémorphe $\dot{a}$ B $P$ si oui, quelles sont leurs propriétés? quel est leur ensemble? Etude du problème analogue pour les algèbres de LrE.

La móthode suivie généralise celle de O. Schrener ("systèmes de facteurs s). Application $\dot{a}$ la détermination des groupes de LIE $\dot{a} \mathrm{n} \leq 4$ dimensions. [Thèse soutenue le 17-4-1951 à l' Jniversité de Strasbourg].

I. - O. Schreier [29] a formulé le problème suivant:

Deux groupes $B$ et $F$ étant donnés, trouver les extensions $E(B, F)$ de $F$ par $B\left({ }^{1}\right)$, c'est-à-dire les groupes $E$ qui admettent $F$ comme sous-groupe distingué tel que $E / F$ soit isomorphe à $B$.

II a montré que toute extension $E(B, F)$ détermine une classe de couples d'applications $(g, \xi)$, couples que nous appellerons cocycles de $B$ à valeurs dans $F$; et que la donnée d' un tel cocycle permet de définir sur l'ensemble produit $B \times F$ une structure d'exstension $E(B, F)$.

De plus, en introduisant une notion d'équivalence entre cocyoles (qui se réduit à la notion de cohomologie selon EILENBERG-MacLane dans le cas où $F$ est abélien), on obtient une correspondance biunivoque entre classes d' équivalence de cocycles de $B$ à valeurs dans $F$ et classes d' équivalence d'exten. sions de $F$ par $B$.

Lorsque $B$ et $F$ sont des groupes topologiques, que nous supposerons tonjours séparés, il est naturel de définir une extension $E(B, F)$ comme un groupe topologique admettant $F$ comme sous-groupe (topologique) fermé distingué, tel que $E / F$ soit topologiquement isomorphe à $B$.

Le problème se pose donc, un cocycle $(g, \xi)$ étant donné, de trouver une topologie sur l' ensemble produit $B \times F$ qui définisse, avec $(g, \xi)$, une extension $E(B, F)$.

Ce ne sera pas toujours possible: mais le résultat de ScHREIER peut se généraliser lorsqu' on impose aux cocycles certaines conditions de continuité et lorsqu' on définit entre eux une équivalence continue.

On peut alors affirmer: il y a correspondance biunivoque entre les classes de cocycles de $B$ à valeurs dans $F$, continus à l'élément neutre de $B$ et continúment équivalents, et les classes d'équivalence des extensions presque-fibrées de $F$ par $B$.

(1) $E(B, F)$ est parfois appele extension de B par $\mathrm{F}$ : je garde la terminologie de Schrbier. 
Des travaux de H. NaGao [26] et N. J. Vilenkin [35] il résulte que, si $B$ et $F$ sont métrisables, toute extension $E(B, F)$ est presque-fibrée, de sorte que cette proposition s'applique à un cas très général.

La notion d'extension presque-fibrée généralise celle d'extension fibrée, c'est-à-dire d'une extension $E(B, F)$ dans laquelle les classes à droite suivant $F$ définissent une structure d'espace fibré de fibre $F$ et de base $B$. Dans la correspondance donnée plus haut, aux classes des extensions fibrées correspondent les classes des cocycles continus an voisinage de l'élément nentre de $B$.

A toute extension $E(B, F)$ est associée une représentation $\chi$ de $B$ dans le groupe quotient du gronpe des automorphismes de $F$ par le sous-groupe des automorphismes intérieurs, que nous appellerons le caractère de $E(B, F)$. Il y a alors lieu d'étudier l'existence d'extensions de caractère donné, et de determiner leur ensemble. Plusieurs auteurs ont donné des critères d'existence dans le cas discret: nous en verrons un autre, qui peut être appliqué aussi dans le cas topologique, moyennant des hypothèses convenables.

Pour les groupes discrets et lorsque $F$ est abélien, on sait donner à l'ensemble des classes d' équivalence des extensions $E(B, F)$ de caractère $\chi$ une structure de groupe, isomorphe au deuxième groupe de cohomologie de $B$ à valeurs dans $F$ relatif à $\chi$. Ce résultat s'étend d'abord au cas où $F$ n'est pas abélien, grâce à la définition des extensions préinessentielles, en suite aux groupes topologiques vérifiant certaines propriétés, qui sont toujours satisfaites si $F$ est un groupe de LIE ou un groupe compact métrisable. Le groupe de cohomologie qu'il faut considérer ici est celui de $B$ à valeurs dans le centre de $F$.

Les extensions préinessentielles peuvent se caractériser comme celles qui admettent un sous-groupe se projetant sur $B$ et ne rencontrant $F$ que dans son centre; pour montrer leur intérèt, rappelons que toute extension centrale est préine sentielle; et que toute extension d' un groupe compact, d'un groupe de LIE semi-simple ou d'un groupe discret par un gronpe connexe est centrale.

Les extensions inessentielles, e'est-à-dire celles qui admettent un sousgroupe dont la projection sur $B$ est un isomorphisme, trouvent une généra. lisation naturelle dans les extensions localement inessentielles, c' est-à-dire les extensions qui admettent un sous-groupe dont la projection sur $B$ est un isomorphisme local.

Lorsque $B$ vérifie certaines conditions de connexion, toute extension localement inessentielle est préinessentielle: il est alors aisé de déterminer leur ensemble. Mais on peut aussi procéder autrement, sans utiliser les cocy. cles: on trouve alors, généralisant un résultat de A. SHapiro [32] concernant les groupes de LIF compacts, que l'ensemble des classes d'équivalence des extensions localement inessentielles de caractère $\chi$ est un groupe isomorphe au groupe quotient $\operatorname{Hom}(\pi, C) / \operatorname{Hom}^{\prime}(\pi, C)$, où $\pi$ est le groupe de PoIrcarä de $B$ et $C$ est le centre de $F ; \chi$ entre dans la détermination du sous-groupe Hom . 
Cette proposition est une conséquence du fait que toute extension loca. lement inessentielle de $F$ par $B$ admet comme groupe revêtement une extension inessentielle de $F$ par le groupe revêtement simplement connexe de $B$.

Si $F$ est localement compact, toute extension inessentielle de $F$ peut ètre obtenue à partir d'une extension particulière, l'holomorphe de $F$, qui joue ainsi le rôle d'extension « universelle».

II. - Ce sont la les principaux résultats de la théorie générale des extensions des groupes topologiques auxquels j'ai abouti; ils sont complétés par de nombreuses propositions concernant surtout la structure des différents types d'extensions. Dans la deuxième partie de ce mémoire j'étudie des cas particuliers ( $(\%)$.

Si $B$ et $F$ sont deux groupes abéliens localement compacts, il n' est pas difficile de montrer, grâce à la théorie de la dualité et aux résultats connus sur les groupes discrets, que, sous des hypothèses peu restrictives, toute extension abélienne est équivalente au groupe produit.

Dans le cas des groupes abéliens de LIE, j'ai pu indiquer toutes les extensions de symbole $E\left(R, R^{n} \times T^{s}\right), E\left(T, R^{n} \times T^{s}\right), E\left(R^{m}, R\right)$ et les extensions centrales de $R^{n} \times T^{s}$ par $R^{m} \times T^{\prime \prime}$.

J'applique ensuite la théorie générale à la détermination de tous les groupes de LIE à $n \leq 4$ dimensions; pour les groupes résolubles cela demande d'assez longs calculs, car il faut en particulier trouver les groupes des automorphismes des groupes résolubles simplement connexes à 3 dimensions.

Pour terminer, j'esquisse rapidement la théorie des extensions des algèbres de LIE ( $\left.{ }^{3}\right)$, utilisant en partie des résuliats de C. Chevalder-S. ErLenberg [11].

III. - Pour ne pas alourdir les énoncés, j'ai toujours ecrit groupe en entendant groupe topologique séparé. De même par isomorphisme, automorphisme etc. j'entends toujours isomorphisme bicontinu, automorphisme bicontỉnu etc.; si la bicontinuité n' est pas demandée j'ajoute le terme algébrique. Par contre le mot représentation et ses composés sont toujours accompagnés, s'il le faut, de l'adjectif continu. La terminologie et les notations sont celles de N. Bourbaki [3], [4].

Que Monsieur Charles Ebresmann, qui a été pour moi dans ces recherches un guide sûr et infatigable, veuille trouver ici l'expression de ma reconnaissance.

(2) Une partie des résultats de ce mémoire a fait l'objet de courtes notes, dont une en collaboration avee M. EHresmand, parues dans les * C. R. Acad. Sci. Paris ^, 228, pag. 1551 et 2:9, pag. 413 ; dans le * Bull. Acad. Sci. Bruxelles », 36, pag. 579; et dans le « Boll. Un. Mat. Italiana », 5, pag. 264 et pag. 286.

(3) J'ai trouvé cité un travail de M. ABE, en japonais, dans lequel les extensions des algèbres de LiE seraient étudiées complètement, mais duquel il m'a oté impossible de trouver même une analyse. En voici la référence: "Zonkoku sijo Sugaku Danwakai ", 226 (1941). 


\section{CHAPITRE I. - GENERAIIIIES}

\section{§ 1. - La cohomologie de Eilenberg-MacLane.}

Pour développer la théorie des extensions des groupes topologiques selon un schéma analogue à celui employé pour les groupes discrets, il est avantageux de génóraliser la cohomologie de EilenBerg-MadLane aux groupes topologiques quelconques; de plus, pour avoir une terminologie cohérente, il est commode de prendre comme groupe des coefficients un groupe non nécessairement abélien. Il faut bien souligner que cette dernière généralisation concerne seulement la terminologie, non la théorie même: que nous utiliserons en nous posant dans le cas où les cochaînes en étude prennent leurs valeurs dans le centre du groupe des coefficients.

Pour tout groupe $G$ nous noterons $\mathfrak{A}(G)$ (resp. $\mathcal{J}(G)$ ) le groupe des automorphismes (resp. des automorphismes intérieurs) bicontinus de $G$. Sauf mention expresse du contraire nous considérerons $\mathscr{G}(G)$ muni de sa topologie discrète. Nous poserons aussi $\mathfrak{E}(G)=\mathfrak{Q}(G) / \mathfrak{J}(G)$.

Definition 1.1. - Deux groupes $\mathrm{B}$ et $\mathrm{F}$ étant donnés, on appelle préreprésentation de $\mathrm{B}$ dans $\mathfrak{A}(\mathrm{F})$ une application $\xi$ de $\mathrm{B}$ dans $\mathfrak{A}(\mathrm{F})$ qui applique l'élément neutre de $\mathrm{B}$ sur l'élément neutre de $\mathfrak{A}(\mathrm{F})$ et telle que sa projection $\chi=q \xi$ dans $\mathscr{Q}(\mathrm{F})$ soit une représentation, oì $q$ désigne l'application canonique de $\mathfrak{A}(\mathrm{F}) \operatorname{sur} \mathcal{E}(\mathrm{F})$.

Si $F$ est abélien nne préreprésentation est donc une représentation, car $\mathfrak{I}\left(F^{\prime}\right)$ est réduit à l'élément neutre.

Si $\xi: x \rightarrow \xi_{x}$ est une préreprésentation de $B$ dans $\mathscr{C}(F)$ et $\chi$ sa projec. tion dans $\mathcal{E}(F)$, notons par $\vartheta_{x}$ la restriction de $\xi_{\infty}$ au centre $C$ de $F$ : c'est un automorphisme de $C$. L'application $x \rightarrow \vartheta_{x}$ de $B$ dans $\mathscr{A}(C)$ est une représentation qui dépend uniquement de $\chi$, car les antomorphismes intérieurs de $F$ laissent invariant chaque élément de $C$. Lorsqu' il n'y aura pas lieu à confusion, nons noterons avec la même lettre $\chi$ la projection dans $\mathscr{G}(F)$ d' une préreprésentation $\xi$ de $B$ dans $\mathfrak{A}(F)$ et la représentation de $B$ dans $\mathfrak{G}(C)$ qu' on en dédnit.

Definition 1.2. - Deux groupes $\mathrm{B}$ et $\mathrm{F}$ étant donnés, on appelle $\mathrm{n}$-cochâ̂ne $\mathrm{f}\left(\mathrm{x}_{1}, \mathrm{x}_{2}, \ldots \mathrm{x}_{\mathrm{n}}\right)$ pour $\mathrm{n} \geq 1$ de $\mathrm{B}$ à valeurs dans $\mathrm{F}$ une application de $\mathrm{B} \times \mathrm{B} \times \ldots \times \mathrm{B}$ (n facteurs) dans $\mathrm{F}$ telle que $\mathrm{f}\left(\mathrm{x}_{1}, \mathrm{x}_{2}, \ldots, \mathrm{x}_{\mathrm{n}}\right)=\mathrm{y}_{0}$ s'il y a un $\mathrm{i}$ tel que $\mathrm{x}^{\mathrm{i}}=\mathrm{x}_{0}$. Une 0 -cochâ̂ne de $\mathrm{B}$ à valeurs dans $\mathrm{F}$ est un élément de $\mathrm{F}$. $x_{0}$ (resp $y_{0}$ ) désigne l' élément neutre de $B$ (resp. de $F$ ).

Une $n$-cochaîne $(n \geq 1)$ sera dite continue au point $\mathrm{x}_{0}$ (resp. localement continue) si elle est continue au point $\left(x_{0}, \ldots, x_{0}\right)$ (resp. dans un voisinage de ce point); une 0-cochaîne sera toujours considerée comme continue. Nous noterons par $C^{n}(B, F)$ l' ensemble des $n$-cochaînes de $B$ à valeurs dans $F$ : 
e' est un groupe avec la loi de composition

$$
(f g)\left(x_{1}, x_{2}, \ldots, x_{n}\right)=f\left(x_{1}, x_{2}, \ldots, x_{n}\right) g\left(x_{1}, x_{2}, \ldots, x_{n}\right) .
$$

Definition 1.3. - Soit $\mathrm{f}$ une $\mathrm{n}$-cochaîne $(\mathrm{n} \geq 1)$ de $\mathrm{B}$ à valeurs dans $\mathrm{F}$ et $\xi$ une préreprésentation de $\mathrm{B}$ dans $\mathfrak{Q}(\mathrm{F})$. La $(\mathrm{n}+1)$-cochaîne définie par

$$
\begin{aligned}
(1.1)(\delta f)\left(x_{1}, x_{2}, \ldots x_{n+1}\right)= & f\left(x_{1}, x_{2}, \ldots, x_{n}\right)^{(-1)^{n}} \prod_{i=1}^{n} f\left(x_{1}, \ldots, x_{i} x_{1+1}, \ldots, x_{n+1}\right)^{(-1) i+1} \\
& \xi_{x_{1}}\left\{f\left(x_{2}, x_{3}, \ldots, x_{n+1}\right)^{-1}\right\}
\end{aligned}
$$

est dite le cobord de $\mathrm{f}$ relatif $\grave{a} \xi \xi$. Une cochaîne dont le cobord relatif $\grave{a} \xi \xi$ est constant (et donc $=\mathrm{y}_{0}$ ) est appelée un cocycle relatif $\grave{a} \xi$. Si $\mathrm{n}=0$ et $\mathrm{f}=\mathrm{y}$, $\mathrm{y} \varepsilon \mathrm{F},(\delta \mathrm{y})(\mathrm{x})=\mathrm{y} \xi_{\mathrm{x}}\left(\mathrm{y}^{-1}\right)$.

L' ensemble des $n$-cocycles (resp. des $n$-cocycles continus au point $x_{0}$, resp. localement continus) relatifs à $\xi$ sera noté $Z^{n}(B, F, \xi)$ (resp. $\mathbf{Z}^{n}(B, F, \xi)$, resp. $\mathfrak{S}^{n}(B, F, \xi)$ et l'ensemble des $n$-cochaines $(n \geq 1)$ qui sont des cobords (resp. des cobords de cochaînes continues au point $x_{0}$, resp. localement continues) relatifs à $\xi$ sera noté $B^{n}(B, F, \xi)$ (resp. $\mathbf{B}^{n}(B, F, \xi)$, resp. $\mathfrak{B}^{n}(B, F, \xi)$ ). $B^{\circ}(B, F, \xi)$ sera par definition réduit à la $O$-cochaine $f=y_{0}$. Lorsque $(x, y) \rightarrow \xi_{x}(y)$ est une application continue en tout point $\left(x_{0}, y\right), y \varepsilon F$, (resp. dans $V \times F, V$ étant un voisinage de $\left.x_{0}\right)$ les éléments de $\mathbf{B}^{n}(B, F, \xi$ ) (resp. $\left.\mathcal{B}^{n}(B, F, \xi)\right)$ sont des cochaînes continues au point $x_{0}$ (resp. localement continues).

DeFINITION 1.4. - F étant supposé abélien, soient $\mathrm{f}$ et $\mathrm{g}$ deux $\mathrm{n}$-cochaînes de $\mathrm{B} \grave{a}$ valeurs dans $\mathrm{F}: \mathrm{f}$ est dit cohomologue à $\mathrm{g}$ par rapport à la représentation $\xi$ de $\mathrm{B}$ dans $\mathfrak{Q}(\mathrm{F})$ s' il existe une $(\mathrm{n}-1)$-cochaîne $\mathrm{h}$ de $\mathrm{B}$ à valeurs dans $\mathrm{F}$ telle que

$$
\begin{gathered}
f\left(x_{1}, x_{2}, \ldots, x_{n}\right)=g\left(x_{1}, x_{2}, \ldots, x_{n}\right) h\left(x_{1}, x_{2}, \ldots, x_{n-1}\right)^{(-1)^{n-1}} \\
\quad \prod_{i=1}^{n-1} h\left(x_{1}, \ldots, x_{i} x_{i+1}, \ldots, x_{n}\right)^{(-1)^{i+1}} \xi_{x_{1}}\left\{h\left(x_{2}, \ldots, x_{n}\right)^{-1}\right\}
\end{gathered}
$$

Si $F$ est abélien il est aisé de vérifier que tout cobord est un cocycle et que $Z^{n}(B, F, \xi)$ et $B^{n}(B, F, \xi)$ sont des sous-groupes de $C^{n}(B, F)$, qui est alors abélien.

Le groupe quotient $H^{n}(B, F, \xi)=Z^{n}(B, F, \xi) / B^{n}(B, F, \xi)$ prend le nom de $\mathrm{n}$-ième groupe de cohomologie de $\mathrm{B}$ à valeurs dans $\mathrm{F}$ relatif $\grave{a} \xi$ (cfr. [17]). Lorsque $(x, y) \rightarrow \xi_{x}(y)$ satisfait les conditions de continuité convenables, on posera de façon analogue $\mathbf{H}^{n}(B, F, \xi)=\mathbf{Z}^{n}(B, \mathrm{~F}, \xi) / \mathbf{B}^{n}(B, \mathrm{~F}, \xi)$ et $\mathscr{H}^{n}(B, F, \xi)=$ $\tilde{S}^{n}(B, F, \xi) / \mathcal{B B}^{n}(B, F, \xi)$.

Pour $n=2$ nous désignerons par $\mathbf{Z}_{0}^{2}(B, F, \xi)$ (resp. $\mathscr{Z}_{0}^{2}(B, F, \xi)$ le sousgroupe de $\mathbf{Z}^{2}(B, F, \xi)$ (resp. de $\mathscr{Z}^{2}(B, F, \xi)$ ) des cocycles $f\left(x_{1}, x_{2}\right)$ tels que, 
pour tout $x_{1} \varepsilon B$, l'application

$$
x_{2} \rightarrow f\left(x_{1}^{-1}, x_{1}\right)^{-1} f\left(x_{1}^{-1}, x_{2}\right) f\left(x_{1}^{-1} x_{2}, x_{1}\right)
$$

est continue au point $x_{0}$ (resp .dans un voisinage de $\left.x_{0}\right)$. $\mathbf{H}_{0}^{2}(B, F, \xi)$ (resp. $\mathscr{H}_{0}^{z}(B, F, \xi)$ ) sera l'image de $\mathbf{Z}_{0}^{2}(B, F, \xi)$ (resp. de $\left.\mathcal{Z}_{0}^{2}(B, F, \xi)\right)$ dans $\mathbf{H}^{2}(B, F$, (resp. dans $\mathfrak{H}^{2}(B, F, \xi)$ ) (').

Enonçons pour plus tard le lemme suivant, dont la démonstration est une simple vérification à partir de (1.1):

LEMne 1.1. - Soient $\mathrm{B}, \mathrm{B}^{\prime}, \mathrm{F}$ trois groupes, $\xi$ une préreprésentation de $\mathrm{B}^{\prime}$ dans $\mathfrak{A}(\mathrm{F}), \psi$ une représentation de $\mathrm{B}$ dans $\mathrm{B}^{\prime}$. L' application $\psi^{*}: \mathrm{C}^{\mathrm{n}}\left(\mathrm{B}^{\prime}, \mathrm{F}\right) \rightarrow \mathrm{C}^{\mathrm{n}}(\mathrm{B}, \mathrm{F})$ définie par

$$
\left(\psi^{*} f\right)\left(x_{1}, x_{2}, \ldots, x_{n}\right)=f\left(\psi\left(x_{1}\right), \psi\left(x_{2}\right), \ldots, \psi\left(x_{n}\right)\right), x_{i} \varepsilon B
$$

est une représentation qui applique cocycles et cobords relatifs à $\xi$ respective. ment sur cocycles et cobords relatifs à $\xi \psi$.

CorollaIre. - Dans les hypothèses du Lemme 1.1 et si F est abélien, par passage aux quotients on obtient de $\psi^{*}$ une représentation de $\mathrm{H}^{\mathrm{n}}\left(\mathrm{B}^{\prime}, \mathrm{F}, \xi\right)$ dans $\mathrm{H}^{\mathrm{n}}(\mathrm{B}, \mathrm{F}, \xi \psi)$.

Nous verrons au $\$ 10$ un cas dans lequel cette représentation est un isomorphisme.

Considérons les éléments $h$ de $Z^{\mathbf{I}}(B, F, \xi)$; on a

$$
(\delta h)\left(x_{1}, x_{2}\right)=h\left(x_{1}\right)^{-1} h\left(x_{1}, x_{2}\right) \xi_{x_{1}}\left\{h\left(x_{2}\right)^{-1}\right\}=y_{0},
$$

c'est-à-dire $\left.h\left(x_{1}, x_{2}\right)=h\left(x_{1}\right) \xi_{x_{1}} \mid h\left(x_{2}\right)\right\}$.

Si $\xi$ est une représentation, les applications $h$ qui vérifient cette relation sont les représentations croisées de $B$ dans $B$ relatives à $\xi(5)$ : pour tout $y \varepsilon F$ fixe, on vérifie que l'application $x \rightarrow y \xi_{x}\left(y^{-1}\right)$ est une représentation croisée qu' on appelle principale. Par défi. nition c'est un 1-cobord : done si $\xi$ est une représentation on a toujours $B^{1}(B, F, \xi) \subset Z^{1}(B, F, \xi)$.

L'ensemble $B_{h}$ des éléments de $B$ tels que $h(x)=y_{0}$ est un sous-groupe de $B$, en général non distingué: d'une façon plus précise on peut montrer le

Lemme 1.2. - Pour que $\mathrm{B}_{\mathrm{h}}$ soit distingué il faut et il suffit que pour $\mathrm{x}^{\prime} \subseteq \mathrm{B}_{h}$. $\xi_{\mathrm{x}^{\prime}}\{\mathrm{h}(\mathrm{x})\}=\mathrm{h}(\mathrm{x})$ pour tout $\mathrm{x} \& \mathrm{~B}\left({ }^{6}\right)$.

On a la

Proposimion 1.1. - Pour qu' un 1-cocycle de $\mathrm{B}$ à valeurs dans $\mathrm{F}$ relatif $\grave{a} \xi \xi$ soit continu, il faut et il suffit qu' il soit continu au point $\mathrm{x}_{0}$.

Done $\mathbf{Z}^{1}(B, F, \xi)^{n}=\mathscr{B}^{1}(B, F, \xi)$.

La démonstration est la même que pour les représentations (cfr. p. ex. [4], chap. $3, \S 2$, Prop.- 13 ).

(4) Dans [7] la définition de $\mathfrak{H}^{2}(B \not F, \xi)$ est incorrecte: de plus ce groupe doit ètre remplacé par $\mathcal{H}_{\hat{\theta}}^{2}(B, F, \xi)$.

(5) Les representations croisées ont été introduites dans [12] et [32].

(6) Comme on voit en considérant les représentations croisées principales, ce Lemme contient le résultat connu suivant: pour qu'un groupe d'automorphismes $H$ du groupe $G$, laissant fixe l'élément $y$, soit distingué dans $\mathscr{Q}(G)$, il faut et il suffit que $H$ laisse fixe aussi tout $\alpha(y)$ pour $\alpha \varepsilon \mathfrak{Q}(G)$. 


\section{\$ 2. - Définitions. Généralités algébriques (7).}

Defintrion 2.1. - Une extension du groupe $\mathrm{F}$ par le groupe $\mathrm{B}$ est définie par la donnée d' un groupe $\mathrm{E}$ qui admet $\mathrm{F}$ comme sous-groupe distingué fermé, et d' un isomorphisme de $\mathrm{E} / \mathrm{F}$ sur $\mathrm{B}$.

Une telle extension sera notée $E(B, F)$; il sera souvent commode d'.dentifier $E / F$ à $B$ par l'isomorphisme donné.

Si $z \varepsilon E(B, F), F \rightarrow z F z^{-1}$ est un automorphisme $k(z)$ de $F:$ on voit aisément que l'application $k: z \rightarrow k(z)$ est une représentation de $E$ dans $\mathfrak{A}(F)$ qui applique $F$ sur $\mathfrak{J}(F)$. Par passage aux quotients, $k$ se projette sur une repré. sentation $\chi$ de $B$ dans $\&(F)$ :

Definition 2.2. - La représentation $\chi$ de $\mathrm{B}$ dans $\mathcal{B}(F)$ définie par l'extension $\mathrm{E}(\mathrm{B}, \mathrm{F})$ s'appelle le caractère de cette extension $\left({ }^{8}\right)$.

Puisque le noyeau de $l$ est le centralisateur de $F$ dans $E$, le noyean de $\chi$ est la projection de ce centralisateur dans $B$.

Tout élément de $E(B, F)$ peut s'écrire sous la forme $y u(x), y \varepsilon F, x \varepsilon B, u$ étant un système de représentants de $B$ dans $E$, e'est-à-dire une application de $B$ dans $E$ vérifiant $u\left(x_{0}\right)=y_{0}$ et $p u(x)=x$ pour tout $x \& B$, si $p$ désigne l'application canonique de $E$ sur $B$ (application composée de la projection canonique de $E$ sur $E / F$ et de l'isomorphisme de celui-ci sur $B$ ).

On aura évidemment

$$
u\left(x_{1}\right) u\left(x_{2}\right)=g\left(x_{1}, x_{2}\right) u\left(x_{1} x_{2}\right)
$$

où $g$ est une application de $B \times B$ dans $F$. Puisque $u\left(x_{0}\right)=y_{0}, g$ est une cochaìne: de plus, l'associativité dans $E$ s'exprime par

$$
u\left(x_{1}\right) g\left(x_{2}, x_{3}\right) u\left(x_{1}\right)^{-1} g\left(x_{1}, x_{2} x_{3}\right)=g\left(x_{1}, x_{2}\right) g\left(x_{1} x_{2}, x_{3}\right) .
$$

Nous noterons $\xi$ l'application composée $k u$, qui est done une préreprésentation de $B$ dans $\mathcal{G}(F)$, dont la projection dans $\mathcal{G}(F)$ est le caractère $\chi$ de $E(B, F): \xi_{x}$ est l'automorphisme $F \rightarrow u(x) F u(x)^{-1}$.

Puisque

$$
u\left(x_{1}\right) u\left(x_{2}\right) y u\left(x_{2}\right)^{-1} u\left(x_{1}\right)^{-1}=g\left(x_{1}, x_{2}\right) u\left(x_{1} x_{2}\right) y u\left(x_{1} x_{2}\right)^{-1} g\left(x_{1}, x_{2}\right)^{-1}
$$

on aura

(2.2) devient

$$
\xi_{x_{1}} \xi_{x_{8}}(y)=g\left(x_{1}, x_{2}\right) \xi_{x_{1} x_{3}}(y) g\left(x_{1}, x_{2}\right)^{-1}
$$

$$
\xi_{x_{1}}\left\{g\left(x_{2}, x_{3}\right)\right\} g\left(x_{1}, x_{2} x_{3}\right)=g\left(x_{1}, x_{2}\right) g\left(x_{1} x_{2}, x_{3}\right)
$$

(7) Pour la partie algébrique de ce paragraphe cfr. [1], [17], [28], [37].

(8) Le caractère est ce que dans [7] j'ai appelé invariant et ce que BAER [1] a appelé Kollektivcharakter. 
ce qui exprime que $g$ est un cocycle relatif à $\xi$. La loi de composition dans $E$ s'écrit:

$$
y_{1} u\left(x_{1}\right) y_{2} u\left(x_{2}\right)=y_{1} u\left(x_{1}\right) y_{2} u\left(x_{1}\right)^{-1} u\left(x_{1}\right) u\left(x_{2}\right)=y_{1} \xi_{x_{1}}\left(y_{2}\right) g\left(x_{1}, x_{2}\right) u\left(x_{1} x_{2}\right) \text {. }
$$

Un autre système de représentants $v$ de $B$ dans $E$ donnera lieu d'une façon analogue à un autre cocycle $f\left(x_{1}, x_{2}\right)$ relatif à une autre préreprésen tation $\vartheta$. Si $v(x)=h(x) u(x)$, on trouve immédiatement les relations

$$
\begin{gathered}
f\left(x_{1}, x_{2}\right)=h\left(x_{1}\right) \xi_{x_{1}}\left\{h\left(x_{2}\right)\right\} g\left(x_{1}, x_{2}\right) h\left(x_{1} x_{2}\right)^{-1} \\
\vartheta_{x}(y)=h(x) \xi_{x}(y) h(x)^{-1} .
\end{gathered}
$$

Nous noterons dorénavant $(g, \xi)$ un 2-cocycle $g$ de $B$ à valeurs dans $F$ relatif à $\xi$ vếrifiant (2.3).

Definition 2.3. - Les cocycles $(\mathrm{g}, \xi)$ de $\mathrm{B}$ à valeurs dans $\mathrm{F}$ déterminés par des systèmes de représentants de $\mathrm{B}$ dans l'extension $\mathrm{E}(\mathrm{B}, \mathrm{F})$ seront dits associés à l'extension $\mathrm{E}(\mathrm{B}, \mathrm{F})$.

Definition 2.4. - Deux cocycles ( $\mathrm{g}$, $\xi$ ) et (f, i) de $\mathrm{B}$ à valeurs dans $\mathrm{F}$ seront dits équivalents $s^{\prime}$ il existe $\mathrm{h} \varepsilon \mathrm{C}^{1}(\mathrm{~B}, \mathrm{~F})$ tel que les relations (2.4) et (2.5) soient satisfaites.

Remarquons que si $F$ est un groupe abélien cette relation d'équivalence n' est autre que la relation de cohomologie entre cocycles.

Proposinton 2.1. - Les cocycles associés à une extension forment une classe d'equivalence.

Par définition même deux cocycles associés à une même extension sont équivalents. Réciproquement si $(f, \vartheta)$ est équivalent au cocycle $(g, \xi)$ associé à $E(B, F)$ et défini par le système de représentants $u$, le système de représentants $x \rightarrow h(x) u(x)$ définit bien $(f, \vartheta)$.

Proposimion 2.2. - B et $\mathrm{F}$ étant deu:c groupes discrets, tout cocycle (g, de $\mathrm{B}$ à valeurs dans $\mathrm{F}$ est associé à une extension $\mathrm{E}(\mathrm{B}, \mathrm{F})$.

En effet on vérifie aisément que la loi de composition

$$
\left(x_{1}, y_{1}\right)\left(x_{2}, y_{2}\right)=\left(x_{1} x_{2}, y_{1} \xi_{x_{1}}\left(y_{2}\right) g\left(x_{1}, x_{2}\right)\right), \quad x_{i} \varepsilon B, \quad y_{i} \varepsilon F
$$

définie sur l'ensemble produit $B \times F$ est une loi d'extension $E(B, F)$.

Definition 2.o. - Deux extensions $\mathrm{E}(\mathrm{B}, \mathrm{F})$ et $\mathrm{E}^{\prime}(\mathrm{B}, \mathrm{F})$ sont dites équiva. lentes s'il y a un isomorphisme $\mathrm{f}$ de $\mathrm{E}$ sur $\mathrm{E}^{\prime}$ dont la restriction $\dot{a} \mathrm{~F}$ et la projection sur $\mathrm{B}$ (identifié à $\mathrm{E} / \mathrm{F}$ et $\dot{a} \mathrm{E}^{\prime} / \mathrm{F}$ ) sont les transformations identiques; f est appelé un isomorphisme d' équivalence de $\mathrm{E}(\mathrm{B}, \mathrm{F}) \operatorname{sur} \mathrm{E}^{\prime}(\mathrm{B}, \mathrm{F})$.

Un isomorphisme d'équivalence de $E(B, F)$ sur $E^{\prime}(B, F)$ transforme donc tout système de représentants de $B$ dans $E$ en un système de représentants de $B$ dans $E^{\prime}$. Ainsi :

LEMme 2.1. - Les cocyc'es associés à deux extensions èquivalentes sont équivalents. 
La Prop. 2.1, la Prop. 2.2 et ce Lemme se résument pour les groupes discrets dans la

Proposition 2.3. - (théorème de Schreier). - B et F étant deux groupes discrets, il $y$ a correspondance biunivoque entre les classes d'équivalence des extensions $\mathrm{E}(\mathrm{B}, \mathrm{F})$ et les classes d'equivalence des cocycles $(\mathrm{g}, \xi)$ de $\mathrm{B}$ à valeurs dans $\mathrm{F}$.

Au $\S 4$ nous pourrons généraliser ce résultat.

Rappelons la suivante

Proposition 2.4. - ([18], thé. 11.1) - B, F el la représentation $\chi$ de $\mathrm{B}$ dans $\mathcal{E}(\mathrm{F})$ étant donnés, s'il $y$ a des cocycles $(\mathrm{g}, \xi)$ de $\mathrm{B}$ à valeurs dans $\mathrm{F}$ tels $q u e q \xi=\chi$, l'ensemble de leurs classes d'équivalence est en correspondance biunivoque avec $\mathrm{H}^{2}(\mathrm{~B}, \mathrm{C}, \chi)$.

$C$ designe le centre de $F$. Pour la démonstration remarquons que, si $\vartheta$ est une préreprésentation de $B$ dans $\mathfrak{A}(F)$ aveo $q \mathfrak{\vartheta}=\chi$, il existe un cocjole $\left(g^{\prime}\right.$, I) équivalent à $(g, \xi)$. Si $(f, \vartheta)$ est donc un autre cocycle, (2.3) montre que $g^{\prime}\left(x_{1}, x_{2}\right) f\left(x_{1}, x_{2}\right)^{-1}$ est un elément de $C$ que nous pouvons noter $h\left(x_{1}, x_{2}\right): h$ est évidemment un élément de $Z^{2}(B, C, \chi)$. La donnée de $(g, \xi)$ permet done de trouver un cocycle $(f, y)$ avec $q^{9}=\chi$ dans chaque classe d'équivalence. Il n'y a plus qu'à vérifier que, si $h$ et $h^{\prime}$ sont cohomologues, $(g h, \xi)$ et $\left.g h^{\prime}, \xi\right)$ sont équivalents et vice-versa.

\section{§3. - La structure topologique de $E\left(B, F^{\prime}\right)$.}

La détermination de la structure topologique d'une extension est en général un problěme très ardu. Il a êté résolu par différents auteurs dans des cas particuliers:

Proposition 3.1. - Pour que toute extension $\mathrm{E}(\mathrm{B}, \mathrm{F})$ possède une des propriétés suivantes, il suffit que $\mathrm{B}$ et $\mathrm{F}$ la possèdent aussi:

a) être séparé.

b) vérifier le premier (resp. te deuxieme) axiome de dénombrabilité ([35]].

c) être complet ([35]]).

d) être localement compact ([29], [35]).

e) être compact (cfr. [10] chap. 2, \& 3, Prop. 1).

f) être connexe (cfr. [10] chap. 2, \& 4, Prop. 2).

Démonstration de $a)$ : soit $\left\{y_{n}\right\}$ le sous-ensemble de $E$ réduit à l'élément neutre. $F$ étant séparé, $\left\{y_{0}\right\}$ est fermé dans $F ; B$ étant séparé, $F$ est fermé dans $E$ et donc $\left\{y_{0}\right\}$ est fermé dans $E$.

Notons que pour que $E(B, F \mid$ possède une des propriétés $a), b), d), e)$ il est aussi nécessaire que $B$ et $F$ la possèdent.

La détermination de la structure topologique de $E(B, F)$ est particuliè. rement simple si $E$ est un espace fibre $\left({ }^{9}\right)$ :

(9) Pour une définition d'espace fibré voir [14]. 
Definition 3.1. - Une extension $\mathrm{E}(\mathrm{B}, \mathrm{F})$ dans laquelle les classes à droite suivant $\mathrm{F}$ définissent une structure d'espace fibré, sera appelée une extension fibrée $\left({ }^{10}\right)$.

On a les propositions suivantes:

Proposition 3.2. - Pour qu' une extension E(B, F) soit fibrée il faut et il suffit $q u$ 'il existe un système de représentants de $\mathrm{B}$ dans $\mathrm{E}$ continu dans un voisinage de $x_{0}$.

Proposition 3.3. - Pour qu' une extension $\mathrm{E}(\mathrm{B}, \mathrm{F})$ ait une structure d'esrace fibré isomorphe à l'espace produit $\mathrm{B} \times \mathrm{F}$, il faut et il suffit qu'il existe un système de représentants continu de $\mathrm{B}$ dans $\mathrm{E}$.

On sait qu'un groupe de LIE est fibré par un sous-groupe fermé: il n'en est pas ainsi pour tout groupe topologique. Considérons en effet une famille infinie de groupes $E_{z}$ chacun isomorphe an gronpe des rotations de la sphère $S_{4}$ à 4 dimensions: chaque $E_{z}$ est une extension fibrée $E_{\alpha}\left(P_{3}, S_{3}\right), P_{3}$ désignant le groupe projectif à 3 dimensions. $E=\Pi E_{z}$ admet comme sous-groupe distingué le produit $S$ d'une famille infinie de groupes chacun isomorphe à $S_{3}$. Vu la topologie de $E$ et la Prop. 3.2, pour que $E$ puisse être fibrée par $S$, il faut qu'il existe un système de représentants $u_{x}$ de $P_{3}$ dans $E_{\alpha}$ continu dans tout $P_{3}$ : or un tel $u_{\alpha}$ n'existe pas (').

Mais on a la

Proposition 3.4. - Dans les cas suivants toute extension $\mathrm{E}(\mathrm{B} ; \mathrm{F})$ est fibrée:

a) $\mathrm{B}$ ou $\mathrm{F}$ discret.

b) F de Lie (Gleason, cité dans [30]).

c) B localement compact, paracompact et localement contractile en un point, F limite projective de groupes de LIE ([2]).

La démonstration de b) et de e) fait intervenir uniquement la structura d'espace fibré principal de $E(B, F)$. La démonstration de a) est triviale si $B$ est discret; si $F$ est discret, $E(B, F)$ est fibrée car l'application eanonique de $E$ sur $B$ est localement un isomorphisme.

La notion d'extension fibrée pent se généraliser:

DEEINITION 3.2. - On appelle presque-fibrée une extension $\mathrm{E}(\mathrm{B}, \mathrm{F})$ admettant un système de représentants u de $\mathrm{B}$ dans $\mathrm{E}$ tel que l'application $\mathrm{b}: \mathrm{yu}(\mathrm{x}) \rightarrow(\mathrm{x}, \mathrm{y})$ de $\mathrm{E}$ sur le groupe produit $\mathrm{B} \times \mathrm{F}$ soit continue au point $\mathrm{y}_{0} \mathrm{u}\left(\mathrm{x}_{0}\right)$ et que $l$ 'application $\mathrm{h}^{-1}$ soit continue au point $\left(\mathrm{x}_{0}, \mathrm{y}_{0}\right)$.

Il est clair que $u$ est alors continu au point $x_{0}$. Réciproquement si $u$ est un système de représentants de $B$ dans l'extension $E(B, F)$ continu au point $x_{0}$, l'application $h^{-1}$ est continue au point $\left(x_{0}, y_{0}\right)$.

(10) La famille d'homéomorphismes $H_{x}$ est la famille des translations à droite de $E$ qui se projettent sur la transformation $x^{\prime} \rightarrow x^{\prime} x$ de $B$. Te gronpe structural $G$ est le groupe des translations à droite de $F$.

(1i) Cet exemple est dù à A. BoreL. 
Si $V \times U$ est un voisinage de $\left(x_{0}, y_{0}\right), h^{-1}(V \times U)=U u(V):$ soit $W$ un voisinage de $y_{0} u\left(x_{0}\right)$ tel que $W \cap F=U$, et tel que $p(W) \subset V, p$ étant l'application canonique de $E$ sur $B$. Si $W_{1}$ est un autre voisinage de $y_{0} u\left(x_{0}\right)$ tel que $W_{1} W_{1}^{-1} \subset W$, puisque $u$ est continu au point $x_{0}$ nons pourons admettre que $u p\left(W_{1}\right) \subset W_{1}:$ si $U_{x}^{\prime}=p^{-1}(x) \cap W_{1}, U_{x}=U_{x}{ }^{\prime} u(x)^{-1}, U_{x}$ est un voisinage de $y_{0}$ dans $F$ et $W_{1}=\bigcup U_{x} u(x)$ pour $x \in p\left(W_{1}\right)$.

D' autre part $U_{x}=U_{x} u(x) u(x)^{-1} \subset W_{1} W_{1}^{-1} \cap F \subset W \cap F=U$. Donc $W_{1} \subset U u(V)=h^{-1}(V \times U)$. Nous avons démontré $\left({ }^{12}\right)$ la

Proposition 3.5. - Une extension $\mathrm{E}(\mathrm{B}, \mathrm{F})$ est presque-fibrée si et seulement si elle admet un système de représentants de $\mathrm{B}$ dans $\mathrm{E}$ continu au point $\mathrm{x}_{0}$.

Soit $f$ une application de l'espace topologique $A$ dans l'extension $E(B, F)$ et $u$ un système de représentants de $B$ dans $E$ : pour tout $a \varepsilon A$ on a $f(a)=f_{1}(a) u\left(f_{2}(a)\right)$, où $f_{2}$ est la projection $p f$ de $f$ dans $B$ et $f_{1}$ est definie par $f_{1}(a)=f(a) u\left(f_{2}(a)\right)^{-1}$. Arec ces notations on a :

CoRolitaire 1. - Une extension $\mathrm{E}(\mathrm{B}, \mathrm{F})$ est presque-fibrée si et seulement si elle admet un système de représentants a tel que, pour tout espace topologique $\mathrm{A}$ et toute application $\mathrm{f}$ de A dans $\mathrm{E}$ avec $\mathrm{f}\left(\mathrm{a}_{0}\right)=\mathrm{y}_{0} \mathrm{u}\left(\mathrm{x}_{0}\right)$, les deux propo. sitions suivantes sont équivalentes:

a) $\mathrm{f}$ est continue au point $\mathrm{a}_{0}$.

b) $\mathrm{f}_{1}$ et $\mathrm{f}_{2}$ sont continues au point $\mathrm{a}_{0}$.

Que la condition est suffisante on voit en posant $A=B, f=u$. Qu' elle est nécessaire, en considérant l'application $h f$.

De démonstration triviale est aussi le

Corollarre 2. - Soit $\mathrm{E}(\mathrm{B}, \mathrm{F})$ une extension presque-fibrée et $\mathrm{f}$ une application de $\mathrm{E}$ dans l'extension $\mathrm{E}^{\prime}(\mathrm{B}, \mathrm{F})$; 'u étant un système de représentants de $\mathrm{B}$ dans $\mathrm{E}$ continu au point $\mathrm{x}_{0}$, supposons que fu soit un système de repré. seniants de $\mathrm{B}$ dans $\mathrm{E}^{\prime}$ : si $\mathrm{f}$ est continue au point $\mathrm{y}_{0} \mathrm{u}\left(\mathrm{x}_{0}\right), \mathrm{E}^{\prime}(\mathrm{B}, \mathrm{F})$ est presquefibrée et l'application $\mathrm{y} f \mathrm{f}(\mathrm{x}) \rightarrow \mathrm{yu}(\mathrm{x})$ est continue au point $\mathrm{y}_{0} \mathrm{fu}\left(\mathrm{x}_{0}\right)$.

Il est bien clair que toute extension fibrée est presque-fibrée: la réci. proque n' est pas vraie, comme le montre l'exemple donné plus haut: si $u_{x}$ est un système de représentants de $P_{3}$ dans $E_{\alpha}$ continu dans un voisinage de $x_{0},\left(u_{\alpha}\right)$ est un système de représentants de $E / S$ dans $E=\Pi E_{x}$ continu au point $\left(x_{0}\right)$.

NaGao a démontré la

Proposition 3.6. - ([26], thé. 2). - Si B et $\mathrm{F}$ vérifient le premier axiome de dénombrabilité, toute extension $\mathrm{E}(\mathrm{B}, \mathrm{F})$ est presque-fibrée.

Rappelons que tout groupe véxifiant le premier axiome de dénombrabi. lité est métrisable, et réciproquement (ctr. [4], chap. 9, $\$$, Prop. 1).

(12) Pour cette démonstration cfr. [26]. 


\section{4. - Extensions presque- fibrées et extensions fbrées équivalentes.}

Nous nous proposons dans ce paragraphe de généraliser la Prop. 2.3.

Lorsqu' un système de représentants $u$ de $B$ dans $E(B, F)$ vérifie des conditions de continuité, il est clair que les applications

$$
\begin{aligned}
& \left(x_{1}, x_{2}\right) \rightarrow g\left(x_{1}, x_{2}\right)=u\left(x_{1}\right) u\left(x_{2}\right) u\left(x_{1} x_{2}\right)^{-1} \\
& x_{2} \rightarrow g\left(x_{1}^{-1}, x_{1}\right)^{-1} g\left(x_{1}{ }^{-1}, x_{2}\right) g\left(x_{1}-x_{2}, x_{1}\right)=u\left(x_{1}\right)^{-} u\left(x_{2}\right) u\left(x_{1}\right) u\left(x_{1}{ }^{-1} x_{2} x_{1}\right) \\
& (x, y) \rightarrow \xi_{x}(y)=u(x) y u(x)^{-1} \quad x_{i} \varepsilon B, y \varepsilon F
\end{aligned}
$$

vérifient aussi des conditions de continuité. Nous poserons la

Definition 4.1. - Un cocycle ( $\mathrm{g}$, $\xi$ ) de $\mathrm{B}$ à valeurs dans $\mathrm{F}$ est dit continu au point $\mathrm{x}_{0}$ (resp. localement continu, resp. continu) si

a) g est une cochaîne continue au point $\left(\mathrm{x}_{0}, \mathrm{x}_{0}\right)$ (resp. dans un voisinage $\mathrm{V} \times \mathrm{V}$ de ce point, resp. dans $\mathrm{B} \times \mathrm{B}$ ).

b) l'application $\mathrm{x}_{2} \rightarrow \mathrm{g}\left(\mathrm{x}_{1}^{-1}, \mathrm{x}_{1}\right)^{-1} \mathrm{~g}\left(\mathrm{x}_{1}^{-1}, \mathrm{x}_{2}\right) \mathrm{g}\left(\mathrm{x}_{1}^{-1}, \mathrm{x}_{2}, \mathrm{x}_{1}\right)$ est, pour tout $\mathrm{x}_{1} \varepsilon \mathrm{B}$, continue au point $\mathrm{x}_{0}$ (resp. dans $\mathrm{V}$, resp. dans $\mathrm{B}$ ).

c) l'application $(\mathrm{x}, \mathrm{y}) \rightarrow \xi_{x}(\mathrm{y})$ est continue en tout point $\left(\mathrm{x}_{0}, \mathrm{y}\right), \quad \mathrm{g} \varepsilon \mathrm{F}$ (resp. dans $\mathrm{V} \times \mathrm{F}$, resp. dans $\mathrm{B} \times \mathrm{F}$ ).

Il est alors évident qu' à toute extension presque-fibrée (resp. fibrée) est associé un cocycle $(g, \xi)$ continu au point $x_{n}$ (resp. localement continu).

Réciproquement tout cocycle $(g, \xi)$ de $B$ à valeurs dans $F$ continu au point $x_{0}$ est associé à une extension presque-fibrée $E(B, F, g, \xi)$ qu' on peut construire de la façon suivante. Sur l'ensemble produit $B \times F$ définis. sons la loi de composition

$$
\left(x_{1}, y_{1}\right)\left(x_{2}, y_{2}\right)=\left(x_{2} x_{2}, y_{1} \xi_{x_{1}}\left(y_{2}\right) g\left(x_{1}, x_{2}\right)\right)
$$

que nous savons être une loi d'extension $(\S 2)$; comme voisinages de $\left(x_{n}, y_{1}\right)$ prenons les ensembles $V \times U$, où $U$ (resp. $V$ ) est un voisinage de $y_{0}$ (resp. de $x_{1}$ ). Les voisinages d'un élement quelconque $(x, y)$ seront obtenus par translation.

$\mathrm{Si}$ (4.1) est compatible ave cette topologie, $E(B, F, g, \xi)$ sera par définition une extension presque-fibrée.

Vérifions que les axiomes de groupe topologique sont satisfaits ([4], chap. $3, \S 1$ ).

$G V^{\prime}{ }-$ Pour tout $V \times U$ il existe $\nabla^{\prime} \times U^{\prime}$ avec $\left(V^{\prime} \times U^{\prime}\right)\left(V^{\prime} \times U^{\prime}\right) \subset V \times U$. Supposons $V_{1}^{2} \subset V, \quad U_{1}^{3} \subset U, g\left(V_{2}, \quad V_{2}\right) \subset U_{1}, \quad \xi_{V_{3}}\left(U_{2}\right) \subset U_{1}:$ posons $V^{\prime}=V_{1} \cap V_{2} \cap V_{3}, U^{\prime}=U_{1} \cap U_{2}$. Alors $\left(V^{\prime} \times U^{\prime}\right)\left(V^{\prime} \times U^{\prime}\right)$ est l' ensemble des eléments donnés par (4.1), si $y_{i} \varepsilon U^{\prime}, x_{i} \varepsilon V^{\prime}$ : il est contenu done dans $V \times U$.

$G V^{\prime}{ }_{I I}$ - Pour tout $V \times U$ il existe $V^{\prime} \times U^{\prime}$ avec $\left(V^{\prime} \times U^{\prime}\right)^{-1} \subset V \times U$.

Puisque $(x, y)^{-1}=\left(x^{-1}, g\left(x^{-1}, x\right)^{-1} \xi_{x^{-1}}\left(y^{-1}\right)\right)$, il suffit de prendre $V^{\prime}=V_{1} \cap V_{2}, \quad U^{\prime}=U_{1} \cap U_{2}$ si $U_{1}^{-2} \subset U, g\left(V_{1}^{-1}, V_{1}\right) \subset U_{1}$ avec $V_{1}^{-1} \subset V$ et $\xi_{V_{3}}-1\left(U_{2}\right) \subset U_{1}$.

$G V_{I I I}^{\prime}-\left(x_{0}, y_{0}\right)$ appartient à tout $V \times U$. 
$G V_{I V}^{\prime}-$ Quel que soit $(x y)$ et $V \times U$ il existe $V^{\prime} \times U^{\prime}$ avec $(x, y)^{-1}$ $\left(V^{\prime} \times U^{\prime}\right)(x, y) \subset V \times U . \quad(x, y)^{-1}\left(V^{\prime} \times U^{\prime}\right)(x, y)$ est l'ensemble des eléments

$\left(x^{-1} x^{\prime} x, g\left(x^{-1}, x\right)^{-1} \xi_{x^{-1}}\left\{y^{-1} y^{\prime} \xi_{x^{\prime}}(y) g\left(x^{\prime}, x\right)\right\} g\left(x^{-1}, x^{\prime} x\right)\right), \quad x^{\prime} \approx V^{\prime}, \quad y^{\prime} \varepsilon J^{\prime}$ $q \mathrm{u}^{\prime}$ on peut aussi ecrire

$\left(x^{-1} x^{\prime} x, g\left(x^{-1}, x\right)^{-1} \xi_{x^{-1}}\left\{y^{-1} y^{\prime} \xi_{x^{\prime}}(y)\right\} g\left(x^{-1}, x\right) g\left(x^{-1}, x\right)^{-1} g\left(x^{-1}, x^{\prime}\right) g\left(x^{-1} x^{\prime}, x\right)\right)$.

Si $h\left(x^{\prime}\right)=g\left(x^{-1}, x\right)^{-1} g\left(x^{-1}, x^{\prime}\right) g\left(x^{-1} x^{\prime}, x\right), h$ est une application continue au point $x_{0}$, d'après la condition b) de la Déf. 4.1.

Soit alors $x^{-1} V_{1} x \subset V, U_{1}^{2} \subset U, h\left(V_{2}\right) \subset U_{2}, g\left(x^{-1}, x\right)^{-1} \xi_{x^{-1}}\left(U_{2}\right) g\left(x^{-1}, x\right) \subset U_{1}$, $y^{-1} U_{3} y y^{-1} \xi_{\mathrm{v}_{3}}(y) \subset U_{2}$. Si $V^{\prime}=V_{1} \cap V_{2} \cap V_{3}$ et $U^{\prime}=U_{1} \cap U_{2} \cap U_{3}$, on roit que $G V^{\prime}{ }_{V}$ est satisfait.

Lorseque le cocycle $(g, \xi)$ est localement continu, l'extension $E(B, F, g, \xi)$ est fibrée d'après le

LEMME 4.1. - Soit $\mathbf{u}$ un système de représentants continu au point $\mathbf{x}_{0}$ de $\mathrm{B}$ dans l' extension $\mathrm{E}(\mathrm{B}, \mathrm{F})$, qui définit le cocycle $(\mathrm{g}, \xi)$ où $\mathrm{g}$ est localement continu. Alors l'extension $\mathrm{E}(\mathrm{B}, \mathrm{F})$ et fibrée.

En effet les voisinages de $u\left(x_{0}\right)$ sont de la forme $U u(V)$ : si $g$ est continu dans $V^{\prime} \times V^{\prime}$, pour $x \varepsilon V^{\prime}$ fixe posons $g_{x}\left(x^{\prime}\right)=g\left(x^{\prime}, x\right): g_{x}^{-1}(U)=V_{1}$ est alors un voisinage de $x_{0}$. Prenons $x^{\prime} \varepsilon V_{1} \cap V=V^{\prime \prime}$ :

$$
u\left(x^{\prime} x\right)=g\left(x^{\prime}, x\right)^{-1} g\left(x^{\prime}, x\right) u\left(x^{\prime} x\right)=g\left(x^{\prime}, x\right)^{-1} u\left(x^{\prime}\right) u(x) .
$$

Supposant $U$ symétrique on a donc $u\left(x^{\prime} x\right) \& U u(V) u(x)$, e' est-ì-dire $u\left(V^{\prime \prime} x\right) \subset$ $\subset U u(V) u(x)$.

Ainsi tout cocycle continu au point $x_{i,}$ (resp. localement continu) est associé à une extension presque-fibrée (resp. fibrée). De plus toute extension presque-fibrée (resp. fibrée) est équivalente à une extension $E(B, F, g, \xi)$ : en effet si $u$ est un système de représentants de $B$ dans $E(B, F)$ continu an point $x_{0}$ (resp. dans un roisinage de $\left.x_{0}\right)$ et $(g, \xi)$ est le cocycle qu' il définit, l'application $y u(x) \rightarrow(x, y)$ de $E(B, F)$ sur $E(B, F, g, \xi)$ est un isomorphisme d'équivalence.

Si $(f, a)$ ent un autre cocycle continu au point $x_{0}$ et équivalent à $(g, \xi)$ (roir (2.4) et (2.5)), l'application $(x, y) \rightarrow(x, y h(x))$, est un isomorphisme d'équivalence algébrique de $E(B, F, f$, y) sur $E(B, F, g, \xi)$. Pour qu' il soit bicontinu, il faut et il suffit que $h$ soit continu an point $x_{0}$; remarquons que dans le cas des extensions fibrées où l' on considère des cocyclès localement continus, la continuité de $h$ au point $x_{n}$ entraine que $h$ est localement continu.

Definition 4.2. - Deux cocycles $(\mathrm{g}, \xi)$ et $(\mathbf{f}$, $)$ de $\mathrm{B} \dot{a}$ valeurs dans $\mathrm{F}$ sont dits continûment équivalents $s^{\prime}$ il $y$ a $\mathrm{h} \in \mathrm{C}^{1}(\mathrm{~B}, \mathrm{E})$ continu au point $\mathrm{x}_{0}$ tel que (2.4) et (2.5) soient satisfaits.

A toute extension presque-fibrée (resp. fibrée) est associée alors d'une façon canonique une classe de cocyoles continus au point $x_{0}$ (resp. localement continus) continùment équivalents: la classe des cocycles définis par des systèmes de 
représentants continus au point $x_{0}$ (resp. dans un voisinage de $x_{0}$ ). Puisqu'à deux extensions équivalentes est ainsi associée la nème classe de cocycles, on peut formuler:

Proposition 4 1. - Il y a une correspondance biunivoque entre les classes d'equivalence des extensions presque-fibrées (resp. fibrées) $\mathrm{E}(\mathrm{B}, \mathrm{F})$ el les classes de cocycles $(\mathrm{g}, \xi)$ de $\mathrm{B}$ à valeurs dans $\mathrm{F}$, continus au point $\mathrm{x}_{0}$ (resp. localement continus), continûment équivalents.

Va la Prop 3.3, nous avons aussi implicitement démontré le

Conollaire 1. - Il y a une correspondance bianivoque entre les classes d'équivalence des extensions $\mathrm{E}(\mathrm{B}, \mathrm{F})$ qui admettent la structure d'espace fibré triviale $\mathrm{B} \times \mathrm{F}$ et les classes de cocycles continus continûment équivalents $(\mathrm{g}, \xi)$ de $\mathrm{B}$ à valeurs dans $\mathrm{F}$.

Remarquons que pour les cocycles continus la condition b) de la Déf. 4.1 est une conséquence de la condition a).

CoRoLlarRe 2. - Il y a une correspondance biunivoque entre les classes d'équivalence des extensions $\mathrm{E}(\mathrm{B}, \mathrm{F}), \mathrm{B}$ étant un groupe discret. et les classes d'équivalénce des cocycles $(\mathrm{g}, \xi)$ de $\mathrm{B} \dot{a}$ valeurs dans $\mathrm{F}$.

En effet, si $B$ est discret, toute extension $E(B, F)$ est fibrée et l'équivalence continue entre cocycles est identique à l'équivalence algébrique.

La condition c) de la Déf. 4.1 est équivalente à une autre, concernant la continuité de $\xi$, lorsqu' on munit $\mathfrak{A}(F)$ d' une topologie convenable. En effet la Prop. 9 de [4], chap. 10, $\$ 2$ devient ici :

Proposition 4.2. - Soit $\mathrm{E}(\mathrm{B}, \mathrm{F})$ une extension du groupe localement compact $\mathrm{F}$. La représentation $\mathrm{k}$ de $\mathrm{E}$ dans $\mathfrak{\mathrm { Q }}(\mathrm{F})$, qui fait correspondre $\grave{a} \mathrm{z} \subseteq \mathrm{E}$ l'automorphisme $\mathrm{F} \rightarrow \mathrm{zFz}^{-1}$, est continue lorsqu' on munit $\mathfrak{Q}(\mathrm{F})$ de la topologie de la convergence compacte.

Par suite on a immédiatement :

Corollatre 1. - Si F est un groupe localement compact et si l'on munit O(F) de la topologie de la convergence compacte, la condition c) de la Déf. 4.1 est équivalente à la condition

$\left.\mathrm{c}_{4}\right)$ La préreprésentation $\xi$ est continue au point $\mathrm{x}_{0}$ (resp. dans $\mathrm{V}$, resp. dans B).

Corollatre 2. - Si F est un groupe localement compact et si l' on munit $\mathfrak{A}(\mathrm{F})$ de la topologie de la convergence compacte, le caractère de $\mathrm{E}(\mathrm{B}, \mathrm{F})$ est une représentation continue de $\mathrm{B}$ dans $\mathcal{\&}(\mathrm{F})$, muni de la topologie quotient $\mathfrak{Q}(\mathrm{F}) / \mathfrak{d}(\mathrm{F})$.

\section{\& 5. - Isomorphismes et automorphismes d'extensions.}

Soient $E(B, F)$ et $E^{\prime}\left(B^{\prime}, F^{\prime}\right)$ deux extensions et $f$ une représentation de $E$ dans $E^{\prime}$. Si $y u(x)$ et $y^{\prime} v\left(x^{\prime}\right)$ sont les éléments de $E$ et resp. de $E^{\prime},(g, \xi)$ et $\left(g^{\prime}, \xi^{\prime}\right)$ les cocycles définis par $u$ et resp. $v$, posons

$$
f(y u(x))=\varphi\{y u(x)\} v[\Psi\{y u(x)\}] .
$$


On a

$$
\begin{aligned}
& \varphi\left\{y_{1} u\left(x_{1}\right)\right\} \xi_{\psi}^{\prime} y_{1} u\left\{\left(x_{1}\right)\right\}\left[\varphi\left\{y_{2} u\left(x_{1}\right)\right\}\right] g^{\prime}\left(\psi\left\{y_{1} u\left(x_{1}\right)\right\}, \quad \psi\left\{y_{2} u\left(x_{2}\right)\right\}\right) \\
& v\left[\psi\left\{y_{1} u\left(x_{1}\right)\right\} \psi\left\{y_{2} u\left(x_{2}\right)\right\}\right]= \\
& =\varphi\left\{y_{1} \xi_{x_{1}}\left(y_{2}\right) g\left(x_{1}, x_{2}\right) u\left(x_{1} x_{2}\right)\right\} v\left[\psi\left\{y_{1} \xi_{x_{1}}\left(y_{2}\right) g\left(x_{1}, x_{2}\right) u\left(x_{1} x_{2}\right)\right\}\right] \text {. }
\end{aligned}
$$

Supposons que $f$ applique $F$ dans $F^{\prime}$ : alors $\psi$, qui est la projection de $f$ dans $B^{\prime}$, s'annulle sur $F$ et est donc une représentation de $B$ dans $B^{\prime}$. La restriction de $\varphi$ à $F$ coincide avec la restriction de $f$ : c'est done une représentation que nous noterons encore $\varphi$. En prenant dans (5.1) $y_{2}=y_{0}$ et $x_{1}=x_{0}$ on obtient

$$
\varphi\left(y_{1}\right) \varphi\left\{u\left(x_{2}\right)\right\}=\varphi\left\{y_{1} u\left(x_{2}\right)\right\} ;
$$

nous poserons $\varphi\{u(x)\}=h\{\psi(x)\}: h$ est bien déterminé si $\psi$ est biunivoque on si $u$ est tel que $\varphi\{u(x)\}=y_{0}{ }^{\prime}$ lors $q u e \psi(x)=x_{n}{ }^{\prime}$, ce que nous supposerons. (5.1) devient alors

$$
\begin{gathered}
\varphi\left(y_{1}\right) h\left\{\psi\left(x_{1}\right)\right\} \xi_{\psi\left(x_{1}\right)}^{\prime}\left[\varphi\left(y_{2}\right) h\left\{\psi\left(x_{2}\right)\right\}\right] g^{\prime}\left(\psi\left(x_{1}\right), \quad \psi\left(x_{2}\right)\right) v\left[\psi\left(x_{1}\right) \psi\left(x_{2}\right)\right]= \\
=-\left\{\left\{y_{1} \xi_{x_{1}}\left(y_{2}\right) g\left(x_{1}, x_{2}\right)\right\} h\left\{\psi\left(x_{1} x_{2}\right)\right\} v\left[\psi\left(x_{1} x_{2}\right)\right] .\right.
\end{gathered}
$$

Si l'on $\mathrm{y}$ fait d'abord $y_{1}=y_{2}=y_{0}$, et en suite $x_{2}=x_{0}$ et $y_{1}=y_{0}$ on trouve

$$
\begin{gathered}
h\left\{\psi\left(x_{1}\right)\right\} \xi_{\psi\left(x_{1}\right)}^{\prime}\left[h\left\{\psi\left(x_{2}\right)\right\}\right] g^{\prime}\left(\psi\left(x_{1}\right), \psi\left(x_{2}\right)\right) h\left\{\psi\left(x_{1} x_{2}\right) 1^{-1}=\varphi\left(g\left(x_{1}, x_{2}\right)\right.\right. \\
h\{\psi(x)\} \xi_{\psi(x)}^{\prime}[\varphi(y)] h|\psi(x)|^{-1}=\varphi\left[\xi_{x}(y)\right]
\end{gathered}
$$

ce qui exprime que les cocycles $(\varphi g, \varphi \xi)$ et $(\bar{g} \psi, \bar{\xi} \psi)$ sont équivalents, si $(\bar{g}, \bar{\xi})$ est la « restriction» à $\psi(B)$ et $\varphi(F)$ de $\left(g^{\prime}, \xi^{\prime}\right)$.

Il est clair que si $f$ est continu, $\varphi$ et $\psi$ le sont aussi; si de plus $u$ et $v$ sont continus au point $x_{0}$, il en sera de même de $h$. Réciproque::ient si $E(B, F)$ et $E^{\prime}\left(B^{\prime}, F^{\prime}\right)$ sont deux extensions presque-fibrées, la donnée des représentations continues $p$ et $\psi$ vérifiant (5.2) avee $u, v, h$ continus au point $x_{n}$ permet de déterminer une représentation continue $f$ qui applique $F$ dans $F^{\prime}$ : il suffit de poser

$$
f(y u(x))=\varphi(y) h \mid \psi(x)\{v\{\psi(x)\} .
$$

Puisque (5.2) montre que dans la classe d'équiralence de $\left(g^{\prime}, \xi^{\prime}\right)$ il y a un cocycle dont la restriction à $\psi(B)$ et $\varphi(F)$ est identique à $(\varphi g, \varphi \xi)$, on aura:

Proposition 5.1. - a) Pour qu'il existe une représentation continue $f$ do $\mathrm{E}(\mathrm{B}, \mathrm{F})$ dans $\mathrm{E}\left(\mathrm{B}^{\prime}, \mathrm{F}^{\prime}\right)$ qui applique. $\mathrm{F}$ dans $\mathrm{F}^{\prime}$, il faut qu'il existe une repré. sentation continue $\varphi$ de $\mathrm{F}$ dans $\mathrm{F}^{\prime}$ et une représentation continue $\psi$ de $\mathrm{B}$ dans $\mathrm{B}^{\prime}$ telles que pour des cocycles $(\mathrm{g}, \xi)$ et $\left(\mathrm{g}^{\prime}, \xi^{\prime}\right)$ associés à $\mathrm{E}(\mathrm{B}, \mathrm{F})$ et resp. $\dot{a}$ $\mathrm{E}^{\prime}\left(\mathrm{B}^{\prime}, \mathrm{F}^{\prime}\right)$, définis par des systèmes de représentants u et resp. $\mathrm{v}$, on ait

$$
\begin{gathered}
\varphi\{u(x)\}=y_{0}^{\prime} \text { si } \psi(x)=x_{0}{ }^{\prime} \\
\varphi g\left(x_{1}, x_{2}\right)=g^{\prime}\left(\psi\left(x_{1}\right), \psi\left(x_{2}\right)\right) \\
\varphi \xi_{x x}=\xi_{\psi(x)}^{\prime} \varphi .
\end{gathered}
$$

b) Si $\mathrm{E}(\mathrm{B}, \mathrm{F})$ et $\mathrm{E}^{\prime}\left(\mathrm{B}^{\prime}, \mathrm{F}^{\prime}\right)$ sont pre que-fibrées pour que $\mathrm{f}$ existe il faut et il suffit que (o.3) soit vérifié avec $\mathrm{a}$ et $\mathrm{v}$ continus aux points $\mathrm{x}_{0}$ et resp. $\mathrm{x}_{0}$. 
Il y a intérêt à poser la

Defintrion 5.1. - On appelle isomorphisme de $\mathrm{E}(\mathrm{B}, \mathrm{F})$ sur $\mathrm{E}^{\prime}(\mathrm{B}, \mathrm{F})$ un isomorphisme de $\mathrm{E}$ sur $\mathrm{E}^{\prime}$ dont la restriction $\grave{a} \mathrm{~F}$ est un automorphisme de $\mathrm{F}$.

Une définition analogue vaudra pour les isomorphismes locaux. En identifiant $B$ à $E / F$ et à $E^{\prime} / F$, d' un isomorphisme (local) de $E(B, F)$ sur $E(B, F)$ on obtient par passage aux quotients un automorphisme (local) de $B$.

La Prop. 5.1 admet la particularisation suivante:

Corollatre. - Soient $\mathrm{E}(\mathrm{B}, \mathrm{F})$ et $\mathrm{E}^{\prime}(\mathrm{B}, \mathrm{F})$ deux extensions presque-fibrées. Pour qu'il existe un isomorphisme de $\mathrm{E}(\mathrm{B}, \mathrm{F})$ sur $\mathrm{E}^{\prime}(\mathrm{B}, \mathrm{F})$, il faut et il suffit $q u$ 'il existe un automorphisme $\varphi$ de $\mathrm{F}$ et un automorphisme $\psi$ de $\mathrm{B}$ tels que, pour des cocycles $(\mathrm{g}, \xi)$ ed $\left(\mathrm{g}^{\prime}, \xi^{\prime}\right)$ associés à $\mathrm{E}(\mathrm{B}, \mathrm{F})$ et resp. à $\mathrm{E}(\mathrm{B}, \mathrm{F})$, définis par des systèmes de représentants continus au point $\dot{\mathrm{x}}_{0}$, on ait

$$
\begin{aligned}
\varphi g\left(x_{1}, x_{2}\right) & =g^{\prime}\left(\psi\left(x_{1}\right), \psi\left(x_{2}\right)\right) \\
\varphi \xi_{x} & =\xi_{\downarrow(x) \varphi}^{\prime} .
\end{aligned}
$$

On a d'autre part la

Proposition 5.2. - Soit f un isomorphisme algébrique de l'extension presque-fibrée $\mathrm{E}(\mathrm{B}, \mathrm{F})$ sur l'extension $\mathrm{E}^{\prime}(\mathrm{B}, \mathrm{F})$. Supposons $\mathrm{f}$ continu, sa restriction $\grave{a} \mathrm{~F}$ et sa projection sur $\mathrm{B}$ bicontinues: alors $\mathrm{f}$ est bicontinu.

C'est une conséquence immédiate du Corollaire 2 à la Prop. 3.5 (cfr. [26], the. 6).

Notons que de (5.1) suit immédiatement la

Proposimion 5.3. - Soient $\mathrm{E}(\mathrm{B}, \mathrm{F})$ et $\mathrm{E}^{\prime}\left(\mathrm{B}^{\prime}, \mathrm{F}^{\prime}\right)$ deux extensions; $s^{2} i l$ n'existe aucune représentation continue de $\mathrm{F}$ sur un sous-groupe distingué de $\mathrm{B}^{\prime}$, toute représentation continue de $\mathrm{E}$ sur $\mathrm{E}^{\prime}$ applique $\mathrm{F}$ sur $\mathrm{F}^{\prime}$.

Donc dans le cas des isomorphismes:

Corolluatre $1{ }^{\left({ }^{13}\right)}$. - Soient B et F deux groupes tels $q u u^{\prime} i l n^{\prime} y$ ait pas de représentations continues de $\mathrm{F}$ sour un sous-groupe distingué de $\mathrm{B}$. Alors si $\mathrm{E}(\mathrm{B}, \mathrm{F})$ et $\mathrm{E}^{\prime}(\mathrm{B}, \mathrm{F})$ sont deux extensions de $\mathrm{F}$ par $\mathrm{B}$, tout isomorphisme de $\mathrm{E}$ sur $\mathrm{E}^{\prime}$ est un isomorphisme de $\mathrm{E}(\mathrm{B}, \mathrm{F})$ sur $\mathrm{E}^{\prime}(\mathrm{B}, \mathrm{F})$.

Si $f_{\text {, et }} f_{2}$ sont deux isomorphismes de $E(B, F)$ sur $E^{\prime}(B, F), f_{1}^{-1} f_{2}$ est un automorphisme de $E(B, F)$ d'après la

DEFINITION 5.2. - Soit l'extension $\mathrm{E}(\mathrm{B}, \mathrm{F})$ : un automorphisme $\mathrm{f}$ du groupe $\mathrm{E}$ dont la restriction à $\mathrm{F}$ est un automorphisme de $\mathrm{F}$, est dit un automorphisme de $\mathrm{E}(\mathrm{B}, \mathrm{F})$. Si la restriction de $\mathrm{f} \dot{a} \mathrm{~F}$ et la projection de $\mathrm{f}$ dans $\mathrm{F} / \mathrm{F}$ sont les transformations identiques, $\mathrm{f}$ est dit un automorphisme d'équivalence.

13) Ce Corollaire ainsi que le Coroll. a la Prop. 5.1 ont été énoncés pour le cas discret dans [20]. 
Ainsi en particulier tout automorphisme intérieur de $E$ est un automorphisme de $E(B, F)$.

Il est clair que, pour que tout automorphisme de $E$ soit un automorphisme de $-E(B, F)$, il faut et il suffit que $F$ soit un sous-groupe caracté. ristique de $E$. La Prop. 5.3 admet done le

Conollaire 2. - S'il n'existe pas de représentations continues du groupe $\mathrm{F}$ sur un sous-groupe distingué du groupe $\mathrm{B}$, toute extension $\mathrm{E}(\mathrm{B}, \mathrm{F})$ admet $\mathrm{F}$ comme sous-groupe caractéristique.

Parallèlement à la Prop. 5.1 on a maintenant la

Proposimion 5.4. - Soit (g, छ) un cocycle associé à l'extension $\mathrm{E}(\mathrm{B}, \mathrm{F})$. Pour que l'automorphisme $\varphi$ de $\mathrm{F}$ et l'automorphisme $\psi$ de $\mathrm{B}$ puissent être respecticenent la restriction $\grave{a} \mathrm{~F}$ et la projection sur $\mathrm{B}$ d'un automorphisme de $\mathrm{E}(\mathrm{B}, \mathrm{F})$. il faut que les cocycles $\left(\varphi \mathrm{g}, \varphi \xi \varphi^{-1}\right)$ et $(g \psi, \xi \psi)$ soient équivalents. Si $\mid g$. $\xi \mid$ est défini par un système de représentants continu au point $\mathrm{x}_{0}, l \alpha$ condition est aussi suffisante.

Il est clair que ce résultat est indépendant du cocyele $(g, \xi)$ choisi.

En posant $g=g^{\prime}$ et $\xi=\xi^{\prime}$ dans $(5.2)$, on obtient facilement des rensei. gnements sur le groupe des automorphismes de $E(B, F)$ (et donc de $E$ si $F$ est un sous-groupe caractéristique). En particulier si $\varphi$ et $\psi$ sont les transformations identiques, on voit que $h$ prend ses valeurs dans le centre $C$ de $F$ lefr. [37], chap. 2, ex. 6) et qu'il est un 1-cocycle de $B$ à valeurs dans $C$ relatif a $\chi$, si $\chi$ est la représentation de $B$ dans $\mathfrak{A}(C)$ induite par $\xi$. De plus, si $h(x)=c \chi_{x}\left(c^{-1}\right), \quad c \varepsilon C$, l'antomorphisme $f$ est intérieur car $c y u(x) c^{-1}=$ $=y c \chi_{x}\left(c^{-1}\right) u(x)$. Ce qui montre aussi qu'à tout 1-cobord coirespond un automorphisme intérieur. Si l'on peut choisir $u$ continu au point $x_{0}$, tout $h$ corre spondant à un automorphisme est continu $\left({ }^{14}\right)$ et à tout $h$ continu correspond un automorphisme. D'autre part pour deux antomorphismes d'équivalence quelconques de $E(B, F)$ on a trivialement $f_{1} f_{2}=f_{2} f_{1}$.

Proposimion 5.5. - Le groupe des automorphismes d'équicalence de l'exten. sion $\mathrm{E}(\mathrm{B}, \mathrm{F})$ de caractère $\chi$ est abélien; si l'extension est presque-fibrée, il y a un isomorphisme de ce groupe sur $\mathscr{Z}^{1}(B, C, \chi)$ qui fait correspondre à $\mathfrak{B}^{\prime}(B, C, \chi)$ les automorphismes intérieurs d'équivalence induits par les éléments de $\mathrm{F}$.

\section{$\S 6$. - Une méthode de construction.}

Soit $E\left(B^{\prime} F\right)$ une extension de $F$ par $B^{\prime}$ : la donnée du groupe $B$ et d'une représentation continue $\psi$ de $B$ dans $B^{\prime}$ permet de construire une extension de $F$ par $B$ que nous appellerons transposée de $E(B, F)$ par $\psi$ et que nous noterous $\psi^{*} E=\psi^{*} E(B, F)$. La construction est calquée sur celle relative aux

(14) En général on peut seulement dire que l'application $u(x) \rightarrow h(x)$ est continne. 
espaces fibrés [14!. Notons par $p$ l'application canonique de $E$ sur $B^{\prime}$; con. sidérons alors dans le groupe produit $B \times E$ le sous-ensemble $\psi^{*} E$ des couples $(x, z), x \varepsilon B, z \varepsilon E$, tels que $\psi(x)=p(z)$. $\psi$ étant une représentation, $\varphi^{*} E$ est un sous-groupe qui contient $F$ (identifié à $\left\{x_{0}\right\} \times F$ ) comme sousgroupe distingué fermé. $\psi^{*} E$ est, dans $B \times E$, l' image réciproque de l'ensemble $B^{\prime \prime}$ des couples $(x, \psi(x)) \varepsilon B \times B^{\prime}$, par l'application $(x, z) \rightarrow(x, p(z)$ '. Comme $B^{\prime \prime}$ est un groupe isomorphe à $B, \psi^{*} E$ est bien une extension $\psi^{*} E(B, F)$; nous écirons en général seulement $\psi^{*} E$, étant bien entendu qu' il s'agit d' nne extension de $F$ par $B$. dont la structure est déterminée par $\psi$ et par $E\left(B^{\prime}, F\right)$.

Si $v$ est un système de représentants de $B^{\prime}$ dans $E$ qui définit le cocyole $\left(g^{\prime}, \xi^{\prime}\right)$, on voit que $u: x \rightarrow(x, v \psi(x))$ est un système de représentants de $B$ dans $\psi^{*} E$ qui définit le cocycle $(g, \xi)$ avec

$$
g\left(x_{1}, x_{2}\right)=g^{\prime}\left(\psi\left(x_{1}\right), \psi\left(x_{2}\right)\right), \quad \xi_{x}=\xi_{\psi(x)}^{\prime} .
$$

Ainsi à tout cocycle $\left(g^{\prime}, \xi^{\prime}\right)$ associé à $E\left(B^{\prime}, F\right)$ correspond un cocycle transposé $(g, \xi)=\psi^{*}\left(g^{\prime} \xi^{\prime}\right)$ associe à $\psi^{*} E$; notons $q u^{\prime}$ en général non tout co. cycle associé a $\psi^{*} E$ est un cocycle transposé.

Si $v$ est continu au point $x_{0}^{\prime}$ (resp. dans un voisinage de $\left.x_{0}^{\prime}\right), u$ est con. tinu au point $x_{0}$ (resp. dans un voisinage de $\left.x_{0}\right)$ : done si $E\left(B^{\prime}, F\right)$ est presque-fibrée (resp. fibrée), il en est de même de $\psi^{*} E$.

Liapplication $\bar{\psi}:(x, z) \rightarrow z$ de $\psi^{*} E$ dans $E$ est une représentation con. tinue dont la restriction à $F$ est la transformation identique et qui, par passage aux quotients, redonne la représentation $\psi$ de $B$ dans $B^{\prime}$. Si $\psi$ est un isomorphisme (local) $\bar{\psi}$ est un isomorphisme (local) d' extension.

Toute représentation continue $f$ de $l^{\prime}$ extension $E(B, F)$ dans l'extension $E^{\prime}\left(B^{\prime}, F^{\prime}\right)$ qui applique $F$ dans $E^{\prime}$ se decompose canoniquement en une représentation continue $f_{1}$ de $E(B, F)$ dans $\psi^{*} E^{\prime}$ et en la représentation continue $\bar{\psi}$ de $\psi^{*} E^{\prime}$ dans $\mathbb{E}^{\prime}\left(B^{\prime}, F^{\prime}\right)$, si $\psi$ est la projection de $f$. La restriction de $f_{1}$ a $F$ coincide ave la restrietion de $f$, et la projection de $f_{1}$ est la transformation identique de $B$, identifié $E / F$ et $\vec{a} \psi^{*} E^{\prime} / F$.

Remarquons $q u^{\prime}$ une représentation $\psi$ non continue permettra de déterminer par la méthode précédente une structure d'extension algébrique, que nous noterons aussi $\psi^{*} E$. Dans ce cas $\psi^{*} E$ est une extension de $F$ par un groupe $\bar{B}$ qui admet une représentation continue biunivoque sur $B$.

\section{Chapitre II. - SUR L' ENSEMBLE DES EX'TENSIONS.}

\section{\& 7. - Critères d'existence.}

Nous n' avons pas encore abordé le problème de l'existence d'extensions $E(B, F), B$ et $F$ étant deux groupes donnés.

Une extension, que nous appellerons l'extension triviale, existe toujours: le groupe produit $B \times F$; la classe des cocycles associés est celle qui cor. 
tient le cocycle constant; le caractère de cette extension est la représentation constante de $B$ dans $\mathcal{E}(F)\left({ }^{15}\right)$.

Pour $\chi=$ const. il $y$ a donc au moins une extension qui admet $\chi$ comme caractère. En est-il de même pour tout autre $\chi$ ?

Enonçons d' abord le lemme suivant, de démonstration évidente:

LEMME 7.1. - Pour qu' un sous-groupe $\mathrm{F}^{\prime}$ de $\mathrm{F}$ soit distingué dans l' extension $\mathrm{E}(\mathrm{B}, \mathrm{F})$ de caractère $\chi$ il faut et il suffit qu' il soit transformé en luimême par tout automorphisme de $\mathrm{F}$, élément du groupe $\mathrm{k}(\mathrm{E})=q^{-1} \chi(\mathrm{B})$.

Rappelons que $k$ est la représentation de $E$ dans $\mathfrak{Q}(F)$ qui $a z \varepsilon E$ fait correspondre l'automorphisme $F \rightarrow z F z^{-1}$, et que $q$ est l'application canonique de $\mathfrak{A}(F)$ sur $\mathscr{G}(F) . k(E)$ a une structure d'extension évidente de $\mathfrak{g}(F)$ par $\chi^{(B)}$.

En particulier le centre $C$ de $F$, sous-gronpe caractéristique de $F$, est. distingué dans $E$ : le groupe quotient $E / C$ a une structure d'extension $E / C(B, F / C)$ bien déterminée.

Proposirion 7.1. - Supposons donnée l'extension $\mathrm{E}(\mathrm{B}, \mathrm{F})$ de caractère $\chi$ et identifions $\mathfrak{d}(\mathrm{F})$ à $\mathrm{F} / \mathrm{C}$. Il y a un isomorphisme canonique d'équivalence algébrique de $\mathrm{E} / \mathrm{C}(\mathrm{B}, \mathrm{F} / \mathrm{C})$ sur la structure d'extension algébrique $\chi^{*} \mathfrak{Q}(\mathrm{F})$ de $\mathfrak{J}(\mathrm{F})$ par B.

En effet si $p$ est l'application canonique de $E$ sur $B$, la représentation $f=(p, k)$ de $E$ dans $B \times \mathfrak{A}(F)$ a comme noyeau précisément $C$ : l'image de $E$ est l'ensemble des couples $(x, \alpha)$ tel que $\chi_{x}=q(\alpha)$. Par definition donc $f(z) \rightarrow(x, h(z))$ avec $x=p(z)$ est un isomorphisme d'équivalence algébrique de $E_{i} C(B, F / C)$ sur $\chi^{*} \mathfrak{Q}(F)$.

Ainsi, si $B$ est discret, on a le

Conollaire 1. - Supposons donnée l'extension $\mathrm{E}(\mathrm{B}, \mathrm{F})$ de caractère $\chi$ sur le groupe discret B. Il y a un isomorphisme canonique d'équivalence de $\mathrm{E} / \mathrm{C}(\mathrm{B}, \mathrm{F} / \mathrm{C})$ sur $\chi^{*} \mathfrak{A}(\mathrm{F})$ (cfr. [1], thé. 2, Coroll. 3).

D'autre part, grace à la Prop. 4.2 et à son Coroll. 2 , on a le

Corollatre 2. - Soit $\mathrm{E}(\mathrm{B}, \mathrm{F})$ une extension de caractère $\chi$ du groupe localement compact $\mathbf{F}$. Si $\mathfrak{J}(\mathbf{F})$ est fermé dans $\mathfrak{A}(\mathrm{F})$, muni de la topologie de la convergence compacte, et isomorphe à $\mathrm{F} / \mathrm{C}$ et si $\mathrm{k}$ est une représentation ouverte de $\mathrm{E}$ dans $\mathfrak{A}(\mathrm{F})$, alors l' isomorphisme canonique d'équivalence algébrique de $\mathrm{E} / \mathrm{C}(\mathrm{B}, \mathrm{F} / \mathrm{C})$ sur $\chi^{*} \mathfrak{d}(\mathrm{F})$ est un isomorphisme d'équivalence.

Notons que $k$ est certainement ouvert si $B, F$ et $\mathfrak{A}(F)$ sont localement compacts et vérifient le deuxième axiome de dénombrabilité ([27], § 19, thé. 13).

Il y a donc des cas où $E / C(B, F / C)$ est entièrement déterminée par le caractère $\chi$ de $E(B, F)$ : au $\S 13$ nóus en verrons d'autres. Lorsqu'il en est ainsi, l'étude de $E(B, F)$ est ramenée à celle de l'extension du groupe abélien $C$ par $\chi^{*} \mathcal{Q}(F)$.

(15) Dn Coroll. à la Prop. 5.1 suit que si une extension est isomorphe à-l'extension triviale, elle lui est aussi équivalente. 
Revenant au cas gẻnéral, la Prop. 7.1 montre qu' une structure algébrique d'extension $E(B, F)$ de caractère $\chi$ existe, si et seulement s'il existe une extension de $C$ par $q^{-1} \chi(B)$ telle que le sous-groupe se projetant sur l'élément neutre de $\chi(B)$ soit algébriquement isomorphe à $F$. On est ainsi conduit all problème suivant: les extensions $E_{1}(B, F)$ et $E_{2}(F, G)$ étant données, trouver nne extension de $G$ par $E_{1}$ qui soit en même temps une extension de $E_{3}$ par $B$, dans le cas particulier on $G$ est le centre de $E_{z}$. Une solution de ce problème à été donnée par EllendBerg-Machane [18], thé. 8.1. En termes de cocycles ce probleme pent se formuler: le cocycle $(g, \xi)$ de $B$ à valeurs dans $F$ etant donné, trouver un cocycle $(f, \vartheta)$ de $B$ à valeurs dans $E_{2}$, dont la projection dans $F$ soit un cocycle equivalent à $(g, \xi)\left({ }^{(6)}\right)$.

Supposons donné $B, F$, $\chi$ et une extension $E^{\prime}(\chi(B), F)$ de caractère $\chi^{\prime}$ : $\chi^{*} E^{\prime}$ est alors nne structure algébrique d'extension de $F$ par $B$ de caractère $\chi^{\prime} \chi$ (cfr. $\left.\$ 6\right)$; donc, pour que $\chi^{*} E^{\prime}$ ait le caractère $\chi$, il fant et il suffit que $\chi^{\prime}=$ transformation identique. Réciproquement une extension $E(B, F)$ de caractère $\chi$ étant donnée, il lui est associé un cocycle $(g, \xi)$, dont la projection dans $F / C$ est un cocycle transposé par $\chi$ d'un cocycle $\left(f^{\prime}, \vartheta^{\prime}\right)$ de. $\chi(B)$ à valours dans $\mathfrak{I}(F)$ : il existe alors un cocycle $(f, \vartheta)$ de $\chi(B)$ à valeurs dans $F$, dont $(g, \xi)$ est le transposé par $\chi$ (et dont $\left(f^{\prime}, W^{\prime}\right)$ est la projection dans $\mathscr{f}(F)$ ).

Nous pouvons formuler la

Proposition 7.2. - Pour $q u^{\prime} i l$ existe une extension $\mathrm{E}(\mathrm{B}, \mathrm{F})$ de caractère $\chi$, $i l$ faut $q u^{\prime} i l$ existe une extension $\mathrm{E}^{\prime}(\chi(\mathrm{B}), \mathrm{F})$ dont le caractère est la transformation identique de $\chi(\mathrm{B})$. Si $\mathrm{B}$ est discret, cette condition est aussi suffisante.

La mème démonstration donne aussi la

Proposition 7.3. - Les groupes $\mathrm{B}$ et $\mathrm{F}$ et la représentation $\chi$ de $\mathrm{B}$ dans $\delta(\mathrm{F})$ étant donnés, supposons $\chi$ continu pour une topologie séparée sur $\mathcal{G}(\mathrm{F})$, compatible avec la structure de groupe. Pour qu' il existe une extension $\mathrm{E}(\mathrm{B}, \mathrm{F})$ de caractère $\chi$, il suffit qu'il existe une extension $\mathrm{E}^{\prime}(\mathrm{X}(\mathrm{B}), \mathrm{F})$ dont le crractère est la transformation identique de $\chi(\mathrm{B})$. Si $\chi$ est une application ouverte et s'il existe un système de représentants $\mathrm{v}$ de $\chi(\mathrm{B})$ dans $\mathrm{B}$ qui est un isomorphisme, la condition est aussi nécessaire.

Si $\chi$ eit ouverte et si $v$ existe, la condition est nécessaire, car le sousgroupe de $E$ se projetant sur $v(\chi(B))$ est bien une extension $E^{\prime}(\chi(B), F)$ dont le caractère est la transformation identique.

Des mèmes considérations suit encore la

Proposition 7.4. - Un groupe F étant donné, munissons \&(F) d'une topologie séparée compatible. $1 l$ y a alors équivalence entre les propositions suivantes: a) Il existe une extension de $\mathrm{F}$ par $\mathcal{E}(\mathrm{F})$ dont le caractère est la transfor. mation identique de $\mathcal{Q}(\mathrm{F})$.

(16) Il serait alors aise de montrer que $(g, \xi)$ est lui-même projection d'un coeyole équivalent $a(f, \vartheta)$. 
b) Pour tout groupe $\mathrm{B}$ et toute représentation continue $\chi$ de $\mathrm{B}$ dans $\Theta(\mathrm{F})$, il existe une extension $\mathrm{E}(\mathrm{B}, \mathrm{F})$ de caractère $\chi$.

BAER ([1], \& 7) a donné un exemple de groupe discret $F$ pour lequel la proposition b) n' est pas vraie.

Dans la suite nous verrons d'autres critères d'existence pour des extensions particulières $\left({ }^{17}\right)$.

\section{\& 8. - Les extensions préinessentielles.}

Pour obtenir, au moins dans des cas particuliers, des renseignements sur l'ensemble des extensions de caractère donné, la Prop. 2.4 suggère de poser les définitions suivantes.

Definition 8.1. - Une exlension $\mathrm{E}(\mathrm{B}, \mathrm{F})$ est dite localement inessentielle (resp. inessentielle) s'il existe un isomorphime local (resp. un isomorphisme) a de $\mathrm{B}$ dans $\mathrm{E}$ qui se projette sur la transformation identique de $\mathrm{B}$. Si $\mathfrak{a}$ n'est pas continu, $\mathrm{E}(\mathrm{B}, \mathrm{F})$ est dite algébriquement localement inessentielle (resp. algébriquement inessentielle) $\left({ }^{18}\right)$.

Renvoyant à plus tard ( $\$ 13$ ) l'étude de ces extensions, donnons ici la proposition suivante, qui est une conséquence immédiate des définitions (cfr. (2.1) et (2.3)):

Proposrion 8.1. - Pour qu' une extension soit algébriquement localement inessentielle (resp. algébriquement inessentielle) il faut et il suffit qu'il lui soit associé un cocycle $(\mathrm{g}, \xi)$ tel que $\mathrm{g}$ soit constant $\left(=\mathrm{y}_{0}\right)$ dans un voisinage de l'élément neutre de $\mathrm{B} \times \mathrm{B}$ (resp. dans tout $\mathrm{B} \times \mathrm{B}$ ). Pour que, de plus, l'extension soit localement inessentielle (resp. inessentielle) il faut que $(\mathrm{g}, \xi)$ soit localement continu.

Definition 8.2. - Une extension $\mathrm{E}(\mathrm{B}, \mathrm{F})$ est dite algébriquement préines sentielle (resp. préinessentielle) si l'extension $\mathrm{E} / \mathrm{C}(\mathrm{B}, \mathrm{F} / \mathrm{C})$ qu' on obtient par passage au quotient est algébriquement inessentielle (resp. inessentielle).

Proposition 8.2. - Pour qu'une extension E.B, F) soit algéoriquement préinessentielle il faut et il suffit qu'il lui soit associè un cocycle $(\mathrm{g}, \xi)$ tel que g prenne ses valeurs dan $\mathrm{C}$. $\xi$ est alors une représentation de $\mathrm{B}$ dans $\mathfrak{A}(\mathrm{F})$.

La suffisance suit de la Prop. 8.1; la nécessité de l'existence d'un isomorphisme algébriqne $v$ de $B$ dans $E / C$, qui est un système de représen* tants. En effet en composant $v$ avec un système de représentants $u$ de $E / C$ dans $E$ on obtient un système de représentants $u v$ de $B$ dans $E$ que définit

(17) Pour les groupes discrets on pent trouver un autre eritère d'existence dans [B4].

(18) Des exemples d'extensions algebriquement inessentielles et non inessentielles sont donnés par les espaces vectoriels $E$ de dimension infinie: si $F$ est un sous-espace vectoricl fermé de $E, E$ est en effet une extension algébriquement équivalente à $E / F \times F$, mais en géneral non homéomorphe a $E / F \times F$. 
un cocycle vérifiant la condition démandée. Que $\xi$ soit une représentation suit de (2.3).

Proposition 8.3. - Pour $q u$ ' une extension $\mathrm{E}(\mathrm{B}, \mathrm{F})$ de caractère $\chi$ soit algébriquement préinessentielle il faut et il suffit qu'il existe une représenta. tion $\xi$ de $\mathrm{B}$ dans $\mathfrak{Q}(\mathrm{F})$ avec $\mathrm{q} \xi=\chi$.

Que cette condition soit nécessaire résulte de la Prop. 8.2 ; pour montrer $q u^{\prime}$ elle est suffisante, considérons le sous-groupe $E^{\prime}=k^{-1} \xi(B)$ de $E, k$ étant la représentation de $E$ dans $\mathfrak{A}(F)$ se projetant sur $\chi . E^{\prime}$ se projette sur $B$; il existe done un système de représentants $u$ de $B$ dans $E$ avec $u(B) \subset E^{\prime}$ qui définit un cocycle $(g, \xi):(2 \cdot 3)$ montre alors que $g$ prend ses valeurs dans $C$.

On peut donc affirmer:

COROLLAIRE. - Si une extension $\mathrm{E}(\mathrm{B}, \mathrm{F})$ de caractère $\chi$ est algébriquement préinessentielle, toute autre extension de $\mathrm{F}$ par $\mathrm{B}$ de même caractère l'est aussi.

Si $q^{-1} \chi(B)$ est une extension inessentielle $\left({ }^{19}\right)$, la structure algébrique d'extension $\chi^{*} \mathfrak{G}(F)$ l'est aussi: vu la Prop. 7.1 on a:

Proposition 8.4. - Pour que les extensions de F par B de caractère $\chi$ soient algébriquement préinessentielles, il suffit que $q^{-1} \chi(B)$ soil une extension inessentielle de $\mathfrak{I}(\mathbf{F})$.

Cette condition n'est pas nécessaire, car $\chi^{*} \mathfrak{Q}(F)$ peut être inessentielle sans que $q^{-1} \chi(B)$ le soit.

En particulier donc si $\mathfrak{A}(F)$ est une extension inessentielle de $\mathfrak{I}(F)$, toutes les extensions de $F$ par un groupe $B$ quelconque seront algébriquèment préinessentielles. De plus il existe alors une représentation de $\mathscr{E}(F)$ dans $\mathfrak{A}(F)$ qui se projette sur la transformation identique de $\mathcal{E}(F)$ : il existe donc une extension préinessentielle de $F$ par $\mathcal{G}(F)$ dont le caractère est la transformation identique. Réciproquement si une telle extension $E(\mathcal{G}(F), F)$ existe, $E_{i} C(\mathcal{G}(F), F / C)$ est inessentielle et donc, grâce à la Prop. 7.1, $\mathfrak{Q}(F)$ est une extension inessentielle de $\mathscr{J}(F)$. Utilisant la Prop. 7.4 on obtient:

COROLLAIRs. - Un groupe F étant donné, il y a équivalence entre les propositions suivantes:

a) $\mathfrak{A}(\mathbb{F})$ est une extension inessentielle de $\mathfrak{J}(\mathbb{E})$.

b) Il existe une extension préinessentielle de $\mathrm{F}$ par $\mathcal{E}(\mathrm{F})$ dont le caractère est la transformation identique de $\mathcal{B}(\mathrm{F})$.

c) Pour tout groupe $\mathrm{B}$ et toute représentation $\chi$ de $\mathrm{B}$ dans $\mathcal{E}(\mathrm{F})$ il existe une structure algébrique d'extension préinessentielle de $\mathrm{F}$ par $\mathrm{B}$ de-caractère $\chi$.

Donnons pour terminer la

Propositron 8.5. - Si le centre $\mathrm{C}$ de $\mathrm{F}$ est discret, toute extension pré. inessentielle $\mathrm{E}(\mathrm{B}, \mathrm{F})$ est localement inessentielle.

(19) $\times(B)$ 6tant un groupe discret, toute extension algébriquement inessentielle sur $\chi(B)$ est inessentielle. 
En effet $E$ est alors localement isomorphe à $E / C(B, F / C)$, extension inessentielle sur $B$. En composant l'isomorphisme de $B$ dans $E / C$ et l'isomor. phisme local de $E / C$ dans $E$, on obtient un isomorphisme local de $B$ dans $E$ qui se projette sur la transformation identique.

\section{§ 9. - L'ensemble des extensions préinessentielles.}

Uné extension tranchée $E(B, F, u)$ est définie par la donnée d'une exten. sion $E(B, F)$ et d' un système de représentants $u$ de $B$ dans $E$.

Deux extensions tranchées $E(B, F, u)$ et $E^{\prime}\left(B, F, u^{\prime}\right)$ seront dites équivalentes si l'application $y u(x) \rightarrow y u^{\prime}(x)$ est un isomorphisme d'équivalence de $E(B, F)$ sur $E^{\prime}(B, F)$; si cette application est continue à l'élément neutre ainsi que sa réciproque, les deux extensions tranchées seront dites semblables. Le cocycle associé à $E(B, F, u)$ est le cocycle défini par $u$ dans $E B, F)$; le caractère de $E(B, F, u)$ est le caractère de $E(B, F)$.

Il est alors clair qu'à denx extensions tranchées équivalentes est associé le mềme cocycle; et que si le même cocycle est associè à deux extensions tranchées semblables, elles sont équivalentes.

Definttion 9.1. - Une extension $\mathrm{E}(\mathrm{B}, \mathrm{F})$ est appelée inversible s' il existe un système de représentants a de $\mathrm{B}$ dans $\mathrm{E}$ tel que

a) $\mathrm{u}\left(\mathrm{x}_{1}\right) \mathrm{u}\left(\mathrm{x}_{2}\right) \mathrm{u}\left(\mathrm{x}_{1} \mathrm{x}_{2}\right)^{-1}$ est un élément du centre $\mathrm{C}$ de $\mathrm{F}$.

b) l'application $\left(\mathrm{u}\left(\mathrm{x}_{1}\right), \mathrm{u}\left(\mathrm{x}_{2}\right)\right) \rightarrow \mathrm{u}\left(\mathrm{x}_{1} \mathrm{x}_{2}\right)$ est continue au point $\left(\mathrm{u}\left(\mathrm{x}_{0}\right), \mathrm{u}\left(\mathrm{x}_{0}\right)\right)$.

c) l'application $\mathrm{u}\left(\mathrm{x}_{1}\right) \rightarrow \mathrm{u}\left(\mathrm{x}_{2}{ }^{-1} \mathrm{x}_{1} \mathrm{x}_{2}\right)$ est continue au point $\mathrm{u}\left(\mathrm{x}_{0}\right)$, pour tout $\mathrm{x}_{\mathrm{q}} \approx B$.

De a) suit que toute extension inversible est algébriquement prëinessentielle; si $(g, \xi)$ est le cocycle défini par $u$, de b) suit que l'application $\left(u\left(x_{1}\right), u\left(x_{2}\right)\right) \rightarrow g\left(x_{1}, x_{2}\right)$ est continue an point $\left(u\left(x_{0}\right)\right.$, $\left.u\left(x_{4}\right)\right)$, car $\left(u\left(x_{1}\right)\right.$, $\left.u\left(x_{2}\right)\right) \rightarrow g\left(x_{1}, x_{2}\right) u\left(x_{1} x_{2}\right)$ est une application continue.

L' existence d' extensions inversibles est assurée par la

Proposition 9.1. - Toute extension préinessentielle presque-fibrée est inversible.

Soit $E(B, F)$ l' extension considérée : puisque $E / C(B, F / C)$ est inessentielle, $E$ admet un sous-group $E^{\prime}$, extension presque-fibrée de $C$ par $B$. Soit $u$ un système de représentants de $B$ dans $E^{\prime}$ continu au point $x_{0}$ : la condition a) est certainement remplie. D'autre part les décompositions

$$
\begin{gathered}
\left(u\left(x_{1}\right), u\left(x_{2}\right)\right) \rightarrow\left(x_{1}, x_{2}\right) \rightarrow x_{1} x_{2} \rightarrow u\left(x_{1} x_{2}\right) \\
u\left(x_{1}\right) \rightarrow x_{1} \rightarrow x_{2}^{-1} x_{1} x_{2} \rightarrow u\left(x_{2}^{-1} x_{1} x_{2}\right)
\end{gathered}
$$

montrent $q$ ue b) et c) le sont aussi, car $x-u(x)$ est une application continue au point $x_{0}$.

Nous dirons qu' une extension tranchée $E(B, F, u)$ est inversible (resp. algébriquement inessentielle, resp. presque-fibrée, resp. fibrée) si $u$ vérifie les 
conditions a)-c) de la Déf. 9.1 (resp. si $u$ est un isomorphisme algébrique, resp. si $u$ est continu au point $x_{0}$, resp. si $u$ est continu au voisinage de $x_{0}$ ).

LEMmE 9.1. - a) Il existe une loi de composition entre extensions tran. chées inversibles, semblables et de même caractère, qui admet (au moins) un élément neutre à droite.

b) Pour cette loi toute extension admet une extension inverse.

c) L'extension composéo de deux extensions presque-fibrées (resp. fibrées) est presque-fibrée (resp. fibrée).

a) Soient $E_{1}(B, F, u)$ et $E_{2}(B, F, v)$ deux extensions tranchées inversibles, semblables et de même caractère $\chi$ et soient $(g, \xi)$ et $(f, \vartheta)$ les cocycles associés. Sur l'ensemble $E_{\mathrm{l}}$ nons allons construire une extension tranchée que nous noterons $\left(E_{1} \odot E_{2}\right)(B, F, u)$ et à laquelle sera associé le cocycle $(g f, \xi)$. Rappelons que $(g f, \xi)$ est bien un cocycle de $B$ a valeurs dans $F$, ear $g$ et $f$ prennent leurs valeurs dans $O$ et $q \xi=q$ \%. Pour voisinages $W$ de l'elément neutre nous prenons les ensembles de $E_{1}$ qui sont des roisinages de $y_{0} u\left(x_{0}\right)$ dans l'extension $E_{1}(B, F)$; les voisinages des autres éléments seront définis par translation. Ainsi $\left(E_{1} \odot E_{2}\right)(B, F, u)$ sera semblable à $E_{1}(B, F, u)$, aura le mème caractère qu' elle, et sera inversible.

La loi de composition est donnée par

$$
y_{1} u\left(x_{1}\right) \odot y_{2} u\left(x_{2}\right)=y_{1} \xi_{1}\left(y_{2}\right) g\left(x_{1}, x_{2}\right) f\left(x_{4}, x_{2}\right) u\left(x_{1} x_{2}\right)
$$

il nous faut démontrer qu' elle est compatible avec la topologie que nous venons de définir.

$G V^{\prime}{ }_{I}$ - Pour tout $W$ il existe $W^{\prime}$ avec $W^{\prime} \odot W^{\prime} \subset W$.

Notons que l'application $h:\left(u\left(x_{1}\right), u\left(x_{2}\right)\right) \rightarrow f\left(x_{1}, x_{2}\right)$ est continue au point $\left(u\left(x_{0}\right), u\left(x_{0}\right)\right)$ : en effet, comme nous l'avons remarqué plus haut, $\left(v\left(x_{1}\right), v\left(x_{2}\right)\right) \rightarrow f\left(x_{1}, x_{2}\right)$ est continue au point $\left(v\left(x_{0}\right), v\left(x_{0}\right)\right)$, et d'autre part l'application $u^{\prime}(x) \rightarrow v(x)$ est continue an point $u\left(x_{0}\right)$. Soit alors $W_{1}$ tel que $W_{1}^{3} \subset W$, et dóterminons $W_{2}$ par la condition $h\left(W_{2} \cap u(B), W_{2} \cap u(B)\right) \subset W_{1}$. $W^{\prime}=W_{1} \cap W_{2}$ satisfait la condition demandée :

$W^{\prime} \odot W^{\prime}=W^{\prime} W^{\prime} h\left(W^{\prime} \cap u(B), W^{\prime} \cap u(B)\right) \subset W_{1}^{3} \subset W$.

$G V^{\prime}{ }_{I I}$ - Pour tout $W$ il existe $W^{\prime}$ tel que $\overline{W^{\prime}} \subset W$, en désignant par $\overline{y u(x)}$ l'inverse de $y u(x)$ pour la loi $\odot$. On a immédiatement $\overline{y u(x)}=$ $=f\left(x^{-1}, x\right)^{-1}(y u(x))^{-1}$. L' application $h^{\prime}: u(x) \rightarrow f\left(x^{-1}, x\right)^{-1}$ est continue au point $u\left(x_{0}\right)$ : prenons $W^{\prime}=W_{1} \cap W_{2}$, où $W_{1} W_{1}^{-1} \subset W$ et $h^{\prime}\left(W_{2} \cap u(B)\right) \subset W_{1}$. Alors $\overline{W^{\prime}}=h^{\prime}\left(W^{\prime} \cap u(B)\right) W^{\prime-1} \subset W$.

$G V^{\prime} I I I-$ Tout $W$ contient $y_{0} u\left(x_{0}\right)$.

$G V^{\prime} r V$ - Quel que soit $y u(x)$ et $W$ il existe $W^{\prime}$ tel que $\left.\overline{y u(x)} \odot W^{\prime} \odot y u_{1}^{\prime} x\right) \subset W$.

Si $y^{\prime} u\left(x^{\prime}\right)$ est un élément de $W^{\prime}$ on a, par un simple caleul qui utilise le fait que $g$ et $f$ sont des cocycles a valeurs dans $C$,

$$
\overline{y u(x)} \odot y^{\prime} u\left(x^{\prime}\right) \odot y u(x)=f\left(x^{-1}, x\right)^{-1} f\left(x^{-1}, x^{\prime}\right) f\left(x^{-1} x^{\prime}, x\right)(y u(x))^{-1} y^{\prime} u\left(x^{\prime}\right) y u(x) \text {. }
$$


Posons $h^{\prime \prime}\left(x^{\prime}\right)$ pour le produit des trois premiers termes de l'expression de droite: dans $E_{2}(B, F, v)$ on a, puisque $f\left(x_{1}, x_{2}\right)=v\left(x_{1}\right) v\left(x_{2}\right) v\left(x_{1} x_{2}\right)^{-1}$, $h^{\prime \prime}\left(x^{\prime}\right)=v(x)^{-1} v\left(x^{\prime}\right) v(x) v\left(x^{-1} x^{\prime} x\right)$. Vu la condition c) de la Déf. 9.1, $v\left(x^{\prime}\right) \rightarrow h^{\prime \prime}\left(x^{\prime}\right)$ est une application continue au point $v\left(x_{0}\right): u\left(x^{\prime}\right) \rightarrow h^{\prime \prime}\left(x^{\prime}\right)$ sera donc une application continue au point $u\left(x_{0}\right)$. Prenons alors $W_{1}^{-}$tel que $W_{1}^{2} \subset W$, $\mathrm{IT}_{2}$ tel que $(\boldsymbol{y} u(x))^{-1} W_{2} y \boldsymbol{u}(\boldsymbol{x}) \subset W_{1}$ et $W_{3}$ tel que $h^{\prime \prime}\left(W_{3} \cap u(B)\right) \subset W_{1}$. Si $W^{\prime}=W_{1} \cap W_{2} \cap W_{3}, G V_{X V}^{\prime}$ est bien satisfait.

Si $E_{2}(B, F, v)$ est une extension tranchée algébriquement inessentielle, on roit que par construction $\left(E_{1} \odot E_{2}\right)(B, F, u)$ est identique à $E_{1}(B, F, u)$. L'existence $d^{\prime}$ ane extension tranchée inessentielle de caractère $\chi$ va suivre de l'existence de l'extension inverse.

b) Soit $E(B, F, u)$ une extension tranchée inversible et $(g, \xi)$ le cocycle associé. $g$ prenant ses valeurs dans le centre de $F,\left(g^{-1}, \xi\right)$ est encore un cocycle de $B$ à valeurs dans $F$. On construira l'extension tranchée inverse $E^{\prime}(B, F, u)$ sur l'ensemble $E$ en prenant comme voisinages de l'élément neutre ceux de $E(B, F)$ et comme loi de composition

$$
y_{1} u\left(x_{1}\right)^{*} y_{2} u\left(x_{2}\right)=y_{1} \xi_{x_{1}}\left(y_{2}\right) g\left(x_{1}, x_{2}\right)^{-1} u\left(x_{1} x_{2}\right)=g\left(x_{1}, x_{2}\right)^{-2} y_{1} u\left(x_{1}\right) y_{2} u\left(x_{2}\right)
$$

Comme tont à l'heure on montre alors que les axiomes $G V^{\prime}{ }^{-} G V^{\prime}{ }_{I V}$ sont satisfaits : on obtient ainsi une extension tranchee inversible, semblable à $E(B, F, u$ et de même caractère.

Il est clair que $\left(E \odot E^{\prime}\right)(B, F, u)$ est une extension tranchée algébriquement inessentielle. Nous avons donc aussi démontré:

Corollatre. - S'il existe une extension inversible $\mathrm{E}(\mathrm{B}, \mathrm{F})$ do caractère $\chi$, il existe aussi une extension de $\mathrm{F}$ par $\mathrm{B}$ algébriquement inessentielle de caractère $\chi$.

c) Si $E_{1}(B, F, u)$ et $E_{2}(B, F, v)$ sont presque-fibrées, il en sera évidemment de même de $\left(E_{1} \odot E_{2}\right)(B, F, u)$. Si l'on part d'extensions tranchées fibrées, l'extension composée sera presque-fibrée et il lui sera associé un cocycle localement continu: le Lemme 4.1 achève la démonstration.

Paisque à chaque classe d'équivalence d'extensions tranchées correspond un cocycle $(g, \xi)$ bien déterminé, il est clair, d'après ce qui précède, que l'ensemble des classes d'équivalence des extensions tranchées inversibles, semblables, auxquelles correspondent des cocycles $(g, \xi)$ avee $\xi$ fixe, forment un groupe isomorphe à un sous-groupe de $Z^{2}(B, C, \chi)\left({ }^{20}\right)$, considéré comme sous-ensemble de $Z^{2}(B, F, \xi)$.

Proposimion 9.2. - L'ensemble des classes d'équivalence des extensions tranchées inversibles et semblables $\mathrm{E}(\mathrm{B}, \mathrm{F}, \mathrm{u})$ auxquelles sont associés des cocycles $(\mathrm{g}, \xi)$ avec $\xi$ fixe est un groupe isomorphe $\grave{a}$ un sous-groupe de

(20) Rappelons (\$ 1) que $\chi$ désigne aussi la représentation de $B$ dans $\mathscr{Q}(C)$ qu'on déduit de $\xi$. 
$\mathrm{Z}^{2}(\mathrm{~B}, \mathrm{C}, \chi)$, si $q^{\xi}=\chi$ Le sous-groupe des classes des extensions presque-fibrées (resp. fibrées) est isomorphe $\grave{a} \mathbf{Z}_{0}^{2}(\mathrm{~B}, \mathrm{C}, \chi)\left(\operatorname{resp} \grave{a} \mathscr{Z}_{0}^{2}(\mathrm{~B}, \mathrm{C}, \chi)\right.$ ).

L' ensemble de tontes les classes d'equivalence des extensions tranchées inversibles, semblables et de même caractère $\chi$ est dono en correspondance biunivoque avec la réunion d'une famille $F$ de sous-groupes de $Z^{2}(B, C, \chi)$ : il ne sera pas en général un groupe, car $\left.\left(E_{1} \odot E_{2}\right) B, F, u\right)$ et $\left(E_{1} \odot E_{2}{ }^{\prime}\right)(B, F, u)$ sont equivalentes si les cocycles associés à $E_{2}(B, F, v)$ et à $E_{2}^{\prime}\left(B, F, v^{\prime}\right)$ ne diffèrent que par la représentation $\mathfrak{F} \neq \mathfrak{Y}^{\prime}$. Comme l'on voit de $(2.4)$ et $(2.5)$ il y a correspondance biunivoque entre les éléments de $\mathscr{F}$ et les 1-cocycles $h$ de $B$ à valeurs dans $F$ relatifs à une représentation $\xi$ quelconque arec $q \xi=\chi$, tels $q$ ue $h(B) c \mid=C$. Donc si $F$ est abélien, fF se réduit à un seul elément:

Corollatre 1. - Si F est un groupe abélien, l'ensemble des classes d'équivalence des extensions tranchées inversibles et semblables $\mathrm{E}(\mathrm{B}, \mathrm{F}, \mathrm{u})$ de même caractère $\chi$ est un groupe isomorphe à un sous-groupe de $\mathrm{Z}^{2}(\mathrm{~B}, \mathrm{~F}, \chi)$.

Si $E_{1}(B, F, u)$ et $E_{1}^{\prime}\left(B, F, u^{\prime}\right)$ (resp. $E_{2}(B, F, v)$ et $E_{2}^{\prime}\left(B, F, v^{\prime}\right)$ ) sont deux extensions tranchées presque-fibrées, $(g, \xi)$ et $\left(g^{\prime}, \xi\right)$ (resp. $(f, \varepsilon)$ et $\left(f^{\prime}, \xi\right)$ ) leurs cocycles, les extensions $E_{1}(B, F)$ et $E_{1}{ }^{\prime}(B, F)$ (resp. $E_{2}(B, F)$ et $\left.E_{2}{ }^{\prime}(B, F)\right)$ sont équivalentes si et seulement si $(g, \xi)$ et $\left(g^{\prime}, \xi\right)$ (resp. $(f, \xi)$ et $\left(f^{\prime}, \xi\right)$ ) sont continûment équivalents. Admettons qu'ils le soient : on voit alors que $(g f, \xi)$ et $\left(g^{\prime} f^{\prime}, \xi_{)}\right.$sont aussi continument equivalents, et donc que $\left(E_{1} \odot E_{2}\right)(B, F)$ et $\left(E_{1}^{\prime} \odot E_{2}^{\prime}\right)(B, F)$ sont deux extensions equivalentes. De plus, si $(g, \xi)$ et $\left(g^{\prime}, \xi\right)$ sont continâment équivalents en tant que cocyeles de $B$ à valeurs dans $F$, de (2.5) on voit que la cochaine $h$ prend ses valeurs dans $C$, et donc que $(g, \chi)$ et $\left(g^{\prime}, \chi\right)$ sont deux cocycles continument equivalents à valeurs dans $C$.

CoRollatre 2. - L'ensemble des classes d'équivalence des extensions $\mathrm{E}(\mathrm{B}, \mathrm{F})$ presque-fibrées (resp. fibrées) préinessentielles, qui admeltent un système de représentants continu au point $\mathrm{x}_{0}$ (resp. dans un voisinage de $\mathrm{x}_{0}$ ) définissant un cocycle $(\mathrm{g}, \xi)$ oì $\xi$ est une représentation donnée avec $\mathrm{q} \xi=\chi$, a une structure de groupe isomorphe à $\mathbf{H}_{0}^{2}(\mathrm{~B}, \mathrm{C}, \chi)$ (resp. $\grave{a} \mathcal{H}_{0}^{2}(\mathrm{~B}, \mathrm{C}, \chi)$ ).

Comme plus haut pour $F$ abelien on a:

Corolluire 3. - F élant un groupe abélien, l'ensemble des classes d'équivalence des extensions $\mathrm{E}(\mathrm{B}, \mathrm{F})$ presque-fibrées (resp. fibrées) de même caractére $\chi$ a une structure de groupe isomorphe à $\mathbf{H}_{0}^{2}(\mathrm{~B}, \mathrm{~F}, \chi)\left(\right.$ resp. $\grave{a} \mathfrak{H}_{0}^{2}(\mathrm{~B}, \mathrm{~F}, \chi)$ ).

Si $B$ et $F$ sont discrets, ce Corollaire 3 redonne le résultat connu de Etrender-Machane $([17], 3.2)$.

Si sealement $B$ est discret, dans toute classe de cocycles continûment equivalents on peut en trouver un avec $\xi$ donné:

CoRolitaIre 4. - B étant un groupe discret, l'ensemble des classes d'équi. valence des extensions $\mathrm{E}(\mathrm{B}, \mathrm{F})$ préinessentielles de même caractère $\chi$ a une structure de groupe isomorphe $\grave{a} \mathrm{H}^{2}(\mathrm{~B}, \mathrm{C}, \chi)$. (Cfr. Prop. 2.4).

Enfin le Corollaire 2 peut se préciser aussi dans le cas suivant: 
CoRolilatRe 5. - Supposons que F soit un groupe localement compact, extension presque-fibrée de son centre $\mathrm{C}$ et supposons $\mathfrak{J}(F)$, muni de la topologie de la convergence compacte, isomorphe à F/C. Alors l'ensemble des classes d'équivalence des extensions $\mathrm{E}(\mathrm{B}, \mathrm{F})$ presque-fibrées (resp. fibrées) préinessentielles de même caractère $\chi$ a une structure de groupe isomorphe à $\mathbf{H}_{0}^{2}(\mathrm{~B}, \mathrm{C}, \chi)$ (resp. à $\mathscr{H}_{0}^{2}(\mathrm{~B}, \mathrm{C}, \chi)$ ).

On voit l'intérêt de ce résultat en se rappelant que les hypothèses sur $F$ sont satisfaites si $F$ est un groupe de LIE connexe, un groupe compact a centre discret on un groupe compacte métrisable.

La démonstration découle du

Lеммe 9.2. - Soit F un groupe vérifiant les hypothèses du Corollaire 5: supposons donnée une extension presque-fibrèe $\mathrm{E}(\mathrm{B}, \mathrm{F})$ et un système de représentan's de $\mathrm{B}$ dans $\mathrm{E}$ continu au point $\mathrm{x}_{0}$ qui définit le cocycle (g, $\xi$ ). Si $\mathrm{E}^{\prime}(\mathrm{B}, \mathrm{F})$ est une autre extension presque-fibrée de même caractère que $\mathrm{E}(\mathrm{B}, \mathrm{F})$, il existe un système de représentants de $\mathrm{B}$ dans $\mathrm{E}^{\prime}$ continu au point $\mathrm{x}_{0}$ qui définit un cocycle $\left(\mathrm{g}^{\prime}, \xi^{\prime}\right)$ avec $\xi^{\prime}=\xi$.

Soit $v$ un système de représentants de $B$ dans $E^{\prime}$ continu au point $x_{n}$ et $(f, \vartheta)$ le cocycle qu'il définit. $\xi$ et $\vartheta$ sont deux applications de $B$ dans $\mathfrak{A}(F)$, continues au point $x_{0}$ pour la topologie de la convergence compacte: $h^{\prime}(x)=\xi_{x} \vartheta_{x}{ }^{-1}$ est une application de $B$ dans $\mathfrak{J}(F)$, que nous identifions a $F / C$, aussi continue au point $x_{0}$. Puisque $F$ est une extension presque-fibrée de son centre, il y a une 1-cochaine $h$ de $B$ à valeurs dans $F$, continue au point $x_{0}$, telle que $h(x) y h(x)^{-1}=h^{\prime}(x)(y)$. Posons alors

$$
\left.g^{\prime}\left(x_{1}, x_{2}\right)=h\left(x_{1}\right) q_{x_{1}} \mid h\left(x_{2}\right)\right\} f\left(x_{1}, x_{2}\right) h\left(x_{1} x_{2}\right)^{-1}
$$

Il est immediat que $\left(g^{\prime}, \xi\right)$ est un cocycle de $B$ à valeurs dans $F$, continument équivalent à $\left(f\right.$, જ) et qui est defini dans $E^{\prime}(B, F)$ par le système de représentants $x \rightarrow h(x) v(x)$ continu au point $x_{0}$.

Il est intéressant de noter que dans certains cas on pent définir une loi de composition entre extensions. vérifiant les propriétés du Lemme 9.1 sans avoir recours à la notion d'extension tranchée et sans s'appuyer sur les cocycles (qui restent par contre nécessaires pour aboutir aux Corollaires 2-5). Il en est ainsi par exemple si le caractère $\chi$ est l'application. constante et si l'on se borne à considérer des extensions presque-fibrées. En effet dans ce cas le centralisateur de $F$ dans une extension $E(B, F)$ est un sous-groupe de symbole $E^{\prime}(B, C)$ (cfr. Prop. 11.3). L'extension composée de $E_{1}(B, F)$ et $E_{2}(B, F)$ s'obtient alors ainsi : le groupe produit $E_{1} \times E_{2}^{\prime}$ est une extension de $F \times O$ par $B \times B$, dont nous notons par $\bar{E}(B, F \times C)$ le plus grand sous-groupe se projetant sur la diagonale de $B \times B$. Le sous groupe $H$ de $E$ des éléments de la forme $\left(c, c^{-1}\right), c \varepsilon C$, est fermé et distingué: $\bar{E} / H$ a une structure évidente d'extension préinessentielle presque-fibrée de $F$ par $B$ dont le caractère est l'application constante. 
Cette construction, qui généralise celle donnée dans [31], peut à son tour se généraliser pour les extensions tranchées. Soient $E_{1}(B, F, u)$ et $E_{2}(B, F: v)$ deux extensions tranchées inversibles, presque-fibrées, de même caractère $\%$ : soit $\bar{E}(B, F \times C, u)$ le sous-groupe de $E_{1} \times E$, des éléments de la forme $\left(y u(x), c v\left(x_{i}\right)\right.$, muni de sa strueture d'extension tranchee de $F \times C$ par $B$. Puisque les extensions dont nous sommes partis ont le même caractère. le sous-groupe $H$ des éléments $\left(c, c^{-1}\right)$ est distingué et fermé dans. $\bar{E}$ et $\bar{E}_{l} H$ a une structure d'extension tranchée inversible, presque-fibrée, de caractère $x$ $q u^{\prime}$ on voit aisément être équivalente à $\left(E_{1} \odot E_{\mathrm{v}}\right)(B, F, u)$.

\section{$\S 10$. Relations entre $E(B, F)$ et $E / C(B, F / C)$.}

Si $B$ est un groupe discret, nous avous vu au $\S 7$ que pour toute extension $E(B, F)$ de caractère $\chi, E / C(B, F / C)$ est équivalente à $\chi^{*} \mathfrak{E}(F)$, après identification de $\mathfrak{I}(F)$ à $F / C$. Ainsi toute extension $E(B, F)$ de carac. tère $\chi$ a aussi une structure de symbole $E\left(\chi^{*} \mathfrak{G}(F), C\right)$. La correspondance $E(B, F) \rightarrow E\left(\chi^{*} \mathfrak{Q}(F), C\right)$ conserve la relation d'equivalence, mais il se peut qu'à des extensions non équivalentes de $F$ correspondent des extensions équiva. lentes de $C$.

ErLenberg-Maolane ont énoncé $\left.{ }^{21}\right)$ la

Proposition 10.1. - ([18], thé, 12.3). - B étant un groupe discret, il y a correspondance biunivoque entre l'ensemble des classes d'équivalence d'extensions $\mathrm{E}(\mathrm{B}, \mathrm{F})$ de caractère $\chi$ aux quelles correspond une même classe d'équivalence d' extensions $\mathrm{E}\left(\chi^{*} \mathfrak{Q}(\mathrm{F}), \mathrm{C}\right)$ et le groupe $\mathrm{H}_{\psi}^{2}(\mathrm{~B}, \mathrm{C}, \chi)$.

$H_{\Downarrow}^{2}(B, C, \chi)$ désigne le noyeau de la représentation de $H^{2}(B, C, \chi)$ dans $H^{2}\left(\chi^{*} \mathfrak{d}(F), C, \chi \psi\right)$ décrite dans le Corollaire au Lemme 1.1, si $\psi$ est l'application canonique de $\chi^{*} \mathfrak{Q}(F)=\chi^{*} \mathfrak{Q}(F)(B, F / C)$ sur $B$.

Nons allons voir que, si les extensions de caractère $\chi$ sont préinessen. tielles, $H_{\psi}^{2}(B, C, \chi)$ se réduit à l'élément neutre.

Lemme 10.1. - Soient $\mathrm{B}, \mathrm{B}^{\prime}, \mathrm{F}$ trois groupes, $\xi$ une préreprésentation de $\mathrm{B}$ dans $\mathfrak{Q}(\mathrm{F})$, $\psi$ une représentation de $\mathrm{B}$ sur $\mathrm{B}^{\prime}$ telle que $\mathrm{B}$ soit une extension algébriquement inessentielle sur $\mathrm{B}^{\prime}$. Si $\psi^{*}$ est la représentation do $\mathrm{C}^{\mathrm{n}}\left(\mathrm{B}^{\prime}, \mathrm{F}\right)$ dans $\mathrm{C}^{\prime \prime}(\mathrm{B}, \mathrm{F})$ qu'on déduit de $\psi$, l' image réciproque de $\mathrm{B}^{\mathrm{n}}\left(\mathrm{B}, \mathrm{F}, \mathrm{E} \mathrm{W}^{\prime}\right)$ par $\psi^{*}$ est $\mathrm{B}^{\mathrm{n}}\left(\mathrm{B}^{\prime}, \mathrm{F}, \xi\right)$.

En effet nous pouvons identifier $f \varepsilon B^{n}\left(B^{\prime}, F, \xi\right)$ avec la restriction de $\psi^{*} f$ à un sous-groupe de $B$, algébriquement isomorphe à $B^{\prime}$.

Corollarre. - Dans les hypothèses du Lemme 10.1 et si de plus F est abélien, par passage aux quotients on déduit de $\psi^{*}$ un isomorphisme de $\mathrm{H}^{\mathrm{n}}\left(\mathrm{B}^{\prime}, \mathrm{F}, \xi\right)$ dans $\mathrm{H}^{\mathrm{n}}(\mathrm{B}, \mathrm{F}, \xi \psi)$.

La Prop. 10.1 admet done bien le

(21) EILENBERG-MAcliane ont enonce cette proposition pour $F$ aussi discret; le Coroll. 2 à la Prop. 4.1 nous permet de laisser tomber cette hypothèse. 
CoROLlaIRE. - $\mathrm{B}$ étant un groupe discret. supposons les extensions $\mathrm{E}(\mathrm{B}, \mathrm{F})$ et $\mathrm{E}^{\prime}(\mathrm{B}, \mathrm{F})$ préinessentielles, de même caractère $\chi$ et non équivalentes: alors $\mathrm{E}\left(\chi^{*} \mathfrak{A}(\mathrm{F}), \mathrm{C}\right)$ et $\mathrm{F}^{\prime}\left(\chi^{*} \mathfrak{G}(\mathrm{F}), \mathrm{C}\right)$ ne sont pas équivalentes.

\section{$\S 11$. - I es extensions centrales.}

Definition 11.1. - Une extension $\mathrm{E}(\mathrm{B}, \mathrm{F})$ est dite centrale si la restriction $\grave{a} \mathrm{~F}$ de tout automorphisme intérieur de $\mathrm{E}$ est un automorphisme intérieur de $\mathrm{F}$.

Cette terminologie est justifiée par la

Proposrnion 11.1. - F'étant un groupe abélien, pour qu'il soit dans le centre de $\mathrm{E}(\mathrm{B}, \mathrm{F})$ il faut et il suffit que $\mathrm{E}(\mathrm{B}, \mathrm{F})$ soit centrale.

En effet si $F$ est dans le centre, tout automorphisme intérieur de $E$ est la transformation identique de $F$, et réciproquement

Corollaire. - Le centre de $\mathrm{F}$ est dans le centre de toute extension centrale de $\mathrm{F}$.

Le groupe $k(E)$ correspondant à l'extension centrale $E(B, F)$ est évidemment identique à $\mathfrak{J}(F)$; réciproquement si $k(E)=\mathfrak{J}(F), E(B, F)$ est centrale. De plus, puisque $\mathfrak{J}(F)$ est une extension inessentielle de lui-mème, on peut appliquer la Prop. 8.4:

Proposirion 11.2. - Une extension $\mathrm{E}(\mathrm{B}, \mathrm{F})$ est centrale si et seulement si son caractère est l'application constante de $\mathrm{B}$ dans $\mathcal{B}(\mathbf{F})$. Toute extension centrale est algébriquement préinessentielle.

Notons qu'alors tont sous-groupe distingué de $F$ est distingué aussi dans $E$ (Lemme 7.1).

Dans une extension centrale $E(B, F)$ toute classe à droite suivant $F$ contient un élément permutable avec tous les élémeits de $F$, car chaque classe engendre tous les automorphismes intérieurs de $F$. La réciproque est aussi vraie, car alors $k(E)=\mathfrak{J}(F)$ :

Proposimion 11.3. - Une extension $\mathrm{E}(\mathrm{B}, \mathrm{F})$ est centrale si et seulement si dans toute classe à droite suivant $\mathrm{F}$ il $y$ " un élément permutable avec chaque élément de F.

Cela pent s'exprimer de deux autres façons:

Corollatre 1. - Une extension $\mathrm{E}(\mathrm{B}, \mathrm{F})$ est centrale si et seulement si le centralisateur $\mathrm{C}_{\mathrm{F}}$ de $\mathrm{F}$ dans $\mathrm{E}$ se projette sur $\mathrm{B}$.

Corollatre 2. - Une extension $\mathrm{E}(\mathrm{B}, \mathrm{F})$ est centrale si et seulement si $\mathrm{E}=\mathrm{FC}_{\mathrm{F}}$.

Rappelons que $C_{F}$ est toujours fermé et distingué dans $E$.

En prenant comme système de représentants de $B$ dans $E$ une application a valeurs dans $C_{F}$, on obtient:

Corollarre 3. - Une extension est centrale si et seulement s'il lui est associé un cocycle (g. $\xi$ ) avec $\xi=$ const.. g prend alors ses valeurs dans le centre $\mathrm{C}$ de $\mathrm{F}$. 
D'où le

Corollaire 4. - Soit $\mathrm{E}(\mathrm{B}, \mathrm{F})$ une extension centrale; $\mathrm{E} / \mathrm{C}(\mathrm{B}, \mathrm{F} / \mathrm{O})$ est algébriquement équivalente à l' extension triviale $\mathrm{B} \times \mathrm{F} / \mathrm{C}$.

En particulier done si $C$ se réduit à l'élément neutre, toute extension centrale de $F$ par $B$ est algébriquement équivalente à $B \times F$. Si de plus tous les automorphismes de $F$ sont intérieurs toute extension de $F$ sera centrale et done algébriquement triviale (efr. [33], thé. 110).

L'intérêt des extensions centrales est mis en évidence par les résultats suivants.

Proposition 11.4. - Soit B un groupe connexe et $\mathrm{F}$ un groupe localement compact tet que $\mathcal{E}(\mathrm{F})$, muni de la topologie de la convergence compacte, soit totalement discontinu. Alors toute extension $\mathrm{E}(\mathrm{B}, \mathrm{F})$ est centrale.

C'est une conséquence du Coroll. 2 à la Prop. 4.2.

On sait que les hypothèses sur $F$ sont satisfaites si $F$ est un groupe de Lite semi-simple ou un group compact ([21], thé. 1).

Du Coroll. 1 à la Prop 5.1 on dédnit sans peine (cfr. $\$ 7$, note $\left({ }^{15}\right)$ ):

LeMMe 11.1. - Une extension localement algébriquement isomorphe (resp. localement isomorphti) à l'extension triviale, lui est aussi localement algébriquement équivalente (resp. localement équivalente).

De démonstration triviale est aussi le

LeMme 11.2. - B étant un groupe connexe, toute extension $\mathrm{E}(\mathrm{B}, \mathrm{F})$ locale. ment algébriquement équivalente (resp. localement équivalentej à l'extension triviale est centrale (resp. et localement inessentielle).

LEMME 11.3. - Soit $\mathrm{B}$ un groupe connexe et $\mathrm{F}$ un groupe compact dont le centre est discret: toute extension $\mathrm{E}(\mathrm{B}, \mathrm{F})$ est localement équivalente $\dot{a}$ l'extension triviale.

Soit $p$ la restriction à $C_{F^{r}}$ de l'application canonique de $E$ sur $B . p$ étant un isomorphisme local, $p^{*} E$ est localement isomorphe à $E(B, F)$; il est d'autre part algébriquement équivalt-nt à $C_{F} \times F$. Puisque $F$ est compact et $C_{F}$ est fermé, $p^{*} E$ est équivalent à $C_{F} \times F$ ([4], chap. 3, $\S 2$, ex. 19). Le Lemme 11.1 achève la démonstration.

Le Coroll. 5 à la Prop. 9.2 devient ainsi :

Proposition 11.5. - B étant un groupe connexce et F un groupe de Lie semi-simple, un groupe de Lie compact ou un groupe compact à centre discret, toutes les extensions $\mathrm{E}(\mathrm{B}, \mathrm{F})$ sont centrales et fibrées et l'ensemble de leurs classes d'équivalence est un groupe isomorphe à $\mathfrak{H}_{0}^{2}(\mathrm{~B}, \mathrm{C}$, const.).

Nous allons voir que ce résultat reste vrai aussi avec d'autres hypothéses.

LEMME 11.4. - Deux extensions $\mathrm{E}(\mathrm{B}, \mathrm{F})$ et $\mathrm{E}^{\prime}(\mathrm{B}, \mathrm{F})$ localement équivalentes ¿̀ l'extension triviale qui sont algébriquement équivalentes, sont équivalentes.

En effet ces extensions admettent deux systèmes de représentants $u$ et resp. $u$ qui sont des isomorphismes locaux et qui définissent les cocycles $\left(g\right.$, const.) et resp. $\left(g^{\prime}\right.$, const. $)$ avec $g=g^{\prime}=$ const. dans un voisinage de 
$\left(x_{0}, x_{0}\right)$. Si $h$ est la 1-cochaîne qui indique que $g$ et $g^{\prime}$ sont cohomologues, nous ponvons la supposer constante, et done continue, dans un voisinage de $x_{\diamond}$. Les deux eocycles sont bien continùment equivalents.

Si $F$ est un gronpe discret, toute extension $E(B, F)$ est localement inessentielle: si de plus $B$ est connexe, $E(B, F)$ est aussi centrale, comme on pent roir en considérant l'application $(x, y) \rightarrow \xi_{x x}(y)$ qui doit être continue dans $V \times F, V$ étant un voisinage de $x_{0}$. Réciproquement si l'application canonique de l'extension $E(B, F)$ sur $B$ est tn isomorphisme local, $F$ est un groupe discret. On est ainsi amené à la

DEFINITION 11.2. - Une extension $\mathrm{E}(\mathrm{B}, \mathrm{F})$ d'un groupe discret $\mathrm{F}$ par un groupe non discret $\mathrm{B}$ est appelée un groupe revêtement de $\mathrm{B}$.

Lorsqu'il sera question de groupes revêtements nous identifierons toujours $E: F$ à $B$.

Proposition 11.6. - Soit B un groupe connexe, $\tilde{\mathrm{B}}$ un groupe revêtement de B. Si $F$ est un groupe tel que toute extension de $\mathrm{F}$ par $\tilde{\mathrm{B}}$ est algébriquement équivalente (resp. équivalente) d̀ l'extension triviale, toute extension $\mathrm{E}(\mathrm{B}, \mathrm{F})$ est centrale (resp. et localement inessentielle).

En effet si $\tilde{p}$ est la projection canonique de $\tilde{B}$ sur $B$, qui est an isomorphisme local, $\tilde{p}^{*} E$ est localement isomorphe à $E(B, F)$. Puisque $\tilde{p}^{*} E$ est algébriquement équivalent (resp. équivalent) à $\tilde{B} \times F$, on peut appliquer les Lemmes 11.1 et 11.2 .

On anra donc, grâce an Lemme 11.4:

Corollatre. - Avec les notations de la Prop. 11.6, si toutes les extensions de $\mathrm{F}$ par $\tilde{\mathrm{B}}$ sont équivalentes à l'extension triviale, l'ensemble des classes d'équivalence des extensions $\mathrm{E}(\mathrm{B}, \mathrm{F})$ est un groupe isomorphe à $\mathfrak{H}_{6}^{2 \prime} \mathrm{B}, \mathrm{C}$, const.).

Ce résultat peut s'appliquer par exemple dans le cas où $B$ et $F$ sont des groupes de LIE compacts, on des groupes de LIE quelconques dont l'un semi-simple.

\section{$\S 12$. Les groupes revêtements.}

Nous nous bornerons à l'étude des groupes revêtements d'un groupe $B$ connexe; les résultats qui vont suivre sont en partie connus, mais, je crois, non publiés.

Nous considérerons comme identiques denx groupes revêtements de $B$ s'ils sont deux extensions équivalentes (ou, grâce au Lemme 11.4, algébri. quement equivalentes). On a alors:

Proposition 12.1. - Un groupe discret $\mathrm{F}$ étant donné, l'ensemble des groupes revêtements du groupe connexe $\mathrm{B}$ de symbole $\mathrm{E}(\mathrm{B}, \mathrm{F})$ a une structure de groupe isomorphe à $\mathfrak{H}_{0}^{2}(\mathrm{~B}, \mathrm{C}$, const. $)$.

La composante connexe de l'élément neutre du groupè revetement $E(B, F)$ est aussi un groupe revêtement $\widehat{B(B}, H), H=\widehat{B} \bigcap F . \quad H$ est univo- 
quement déterminé, et il est dans le centre de $F$; en effet il est engendré par $g(B \times B)$, si ( $g$, const.) est le cocycle defini par un système de représentant, de $B$ dans $\widehat{B}$. De plus, puisque $E(B, F)$ est centrale, $H$ se trouvera dans le centre de $E$. $E$ admet $\widehat{B}$ comme sous-groupe distingué et $E \widehat{B}$ est isomorphe à $F / H$. D'autre part $E / H$ est le groupe revêtement trivial $B \times F / H$ et done $E$ a aussi une structure de symbole $E(B \times F / H, H)$.

Lemure 12.1. - Tout groupe revêtement $\mathrm{E}(\mathrm{B}, \mathrm{F})$ d' un groupe connexe $\mathrm{B}$ détermine univoquement un sous-groupe $\mathrm{H}$ du centre de $\mathrm{F}$, et admet un sousgroupe connexe distingué $\widehat{\mathrm{B}}$ extension de $\mathrm{H}$ par $\mathrm{B}$. $\mathrm{H}$ est dans le centre de $\mathrm{E}$ et $\mathrm{E} / \mathrm{H}$ est le groupe revêtement trivial $\mathrm{B} \times \mathrm{F} / \mathrm{H}$.

Proposition 12.2. - Soit $\mathrm{B}$ un groupe connexe admettant un espace revêtement $\left({ }^{22}\right)$ simplement connexe $\left({ }^{23}\right): \dot{\alpha}$ tout sous-groupe $\pi^{\prime} d u$ groupe de Poincaré $n\left({ }^{24}\right)$ de $\mathrm{B}$ correspond un groupe revêtement connexe de $\mathrm{B}$, extension de $\pi / \pi^{\prime}$ par $B$. Si de plus $\mathrm{B}$ est localement connexe, dans tout groupe revêtement connexe $\widehat{\mathrm{B}}(\mathrm{B}, \mathrm{H})$. $\mathrm{H}$ est isomorphe $\grave{a}$ con groupe quotient $\pi / \pi^{\prime}$ : $\grave{a}$ chaque sous-groupe $\pi^{\prime}$ correspond un seul groupe revêtement connexe $\widehat{\mathrm{B}}\left(\mathrm{B}, \pi / \pi^{\prime}\right)$. (cfr. [13], pag. 40).

En effet on démontre (cfr. [10], chap. 2, \& 8, Prop. 5; aussi [8']) qu'on pent donner au revêtement simplement connexe une et une seule structure de groupe revêtement connexe $\tilde{B}$, extension de $\pi$ par $B$. Pour tout sous-groupe $\pi^{\prime}$ on aura done, par passage an quotient, le groupe revètement connexe $\tilde{B} / \pi^{\prime}\left(B, \pi / \pi^{\prime}\right)$. La dernière partie de la proposition est une conséquence da fait que toute représentation locale continue ouverte d'un groupe simplement connexe dans un autre groupe peut se prolonger en une représentation continue ouverte, si elle est définie dans un voisinage connexe de l'élément neutre ([10], chap. 2 , 7 , thé. 3). Cela étant, puisque $\widehat{B(B, H})$ est localement isomorphe à $\widetilde{B}(B, \pi)$, et puisqu' on peut supposer que l'isonorphisme local est défini dans un voisinage connexe, on peut affirmer que $\widehat{B}$ est un groupe quotient $\tilde{B} / K$ de $\tilde{B}$, où $K$ doit ètre contenu dans $\pi$.

Enfin considérons les deux groupes revêtements $\left.\widehat{B(B}, \pi / \pi^{\prime}\right)$ et $\widehat{B^{\prime}}\left(B, \pi / \pi^{\prime}\right)$ : ils sont identiques, puisqu' ils ne sont autre que $\tilde{B} / \pi^{\prime}\left(B, \pi / \pi^{\prime}\right)$.

Pour plus tard énoncons le

LEMME 12.2. - Soit $\tilde{\mathrm{B}}$ un groupe revêtement du groupe $\mathrm{B}$ et $\mathrm{E}(\mathrm{B}, \mathrm{F})$ une extension sur B. Si $\tilde{\mathrm{p}}$ désigne la projection canonique de $\tilde{\mathrm{B}}$ su. $\mathrm{B}, \tilde{\mathrm{p}} * \mathrm{E}$ admet une structure de groupe revêtement de $\mathrm{E}$.

(2z) Un (espace) revètement de l'espace connexe $B$ est un espace fibré de fibre discrète et de base $B$ (cfr. [10] et [14]).

(23) Un espace $E$ est simplement connexe s'il n'admet aucun revètement connexe non isomorphe au revètement constitué par $E$ lui même.

(24) Défini comme le groupe des automorphismes du revêtement simplement connexe. 
En effet il y a une représentation de $\tilde{p}^{*} E$ sur $E$ dont la restriction à un voisinage de l'élément neutre est un isomorphisme local. Celui-ci étant mème un isomorphisme local d'extension, on a le

CorollaIRE. - Si $\tilde{\mathrm{p}}^{* \mathrm{E}}$ est localement inessentielle, il en est de même de $\mathrm{E}(\mathrm{B}, \mathrm{F})$.

\section{\$ 13. - Les extensions localement inessentielles et les extensions inessentielles.}

Nous nous proposons d'étudier ici les notions introduites avec la Déf. 8.1. Des Prop. 3.2 et 3.3 suit d'abord:

Proposition 13.1. - Toute extension localement inessentielle est fibrée; la structure d'espace fibré d'une extension inessentielle est isomorphe à celle de l' espace produit.

Les résultats du $\S 4$ et la Prop. 8.1 donnent:

Propositror 13.2. - Il y a une correspondance biunivoque entre les classes d'équivalence des extensions localement inessentielles (resp. inessentielles) $\mathrm{E}(\mathrm{B}, \mathrm{F})$ et les classes de cocycles de $\mathrm{B}$ à valeurs dans $\mathrm{F}$ localement continus, continûments équivalents, contenant un cocycle $(\mathrm{g}, \xi)$ avec $\mathrm{g}=$ const. au voisinage de $\left(\mathrm{x}_{0}, \mathrm{x}_{0}\right)$ (resp. avec $\mathrm{g}=$ const.).

Done en particulier:

Corollatre 1. - Pour $q w^{\prime}$ il existe une extension inessentielle $\mathrm{E}(\mathrm{B}, \mathrm{F})$ de caractère $\chi$ il faut et il suffit $q u^{\prime} i l$ existe une représentation $\xi$ de $\mathrm{B}$ dans $\mathfrak{G}(\mathrm{F})$ qui se projette sur $\chi$ et telle que l'application $(\mathrm{x}, \mathrm{y}) \rightarrow \xi_{x}(\mathrm{y})$ soit une application continue de $\mathrm{B} \times \mathrm{F}$ dans $\mathrm{F}$.

Dans ce cas particulier la construction du $\& 4$ revient à définir sur l'espace produit $B \times F$ la loi de composition

$$
\left(x_{1}, y_{1}\right)\left(x_{2}, y_{2}\right)=\left(x_{1} x_{2}, y_{1} \xi_{x_{1}}\left(y_{2}\right)\right)
$$

Ce critère d'existence et les résultats des $\S \S 8$ et 9 permettent de con. nattre l'ensemble des extensions de caractère $\chi$. D'autre part, grăce à la Prop. 4.2, il admet la particularisation suivante:

Corollaire 2. - F étant un groupe localement compact, munissons $\mathfrak{E}(\mathbb{F})$ de la topologie de la convergence compacte et $\mathcal{G}_{\mathbf{F}} \mathbf{\mathrm { F }}$ de la topologie quotient. Pour qu'il existe une extension $\mathrm{E}(\mathrm{B}, \mathrm{F})$ de caractère $\chi$ il faut et il suffit qu'il existe un relèvement $\xi$ de $\chi$ dans $\mathfrak{G}(\mathrm{F})$.

La question se pose si l'extension définie par (13.1) est entièrement déter. minée par son caractère $\chi$. Il en sera sûrement ainsi si $F$ est abélien, car alor's $\chi=\xi$, ou si $F$ vérifie les hypothèses du Lemme 9.2, come on voit de sa démonstration:

Proposition 13.3. - Supposons que F soit un groupe abélien, ou un groupe localement compact, extension presque-fibrée de son centre $\mathrm{O}$ et tel que $\mathfrak{I}(\mathbf{F})$, 
muni de la topologie de la convergence compacte, soit isomorphe à $\mathrm{F} / \mathrm{C}$. Alors, pour tout groupe $\mathrm{B}$, deux extensions inessentielles $\mathrm{E}(\mathrm{B}, \mathrm{F})$ et $\mathrm{E}^{\prime}(\mathrm{B}, \mathrm{F})$ de même caractère sont équivalentes.

Ce résultat permet de renforcer la Prop. 7.1:

Corollatre. - Soit $\mathrm{E}(\mathrm{B}, \mathrm{F})$ une extension préinessentielle. Si F C vérifie les hypothèses de la Prop. 13.3 énoncées pour $\mathrm{F}, \mathrm{E} / \mathrm{C}(\mathrm{B}, \mathrm{F} / \mathrm{C})$ est entièrement déterminée par le caractère de $\mathrm{F}(\mathrm{B}, \mathrm{F})$.

$L$ 'étude des extensions localement inessentielles est particulièrement intéressant dans le cas où $B$ admet un groupe revêtement simplement connexe.

Proposition 13.4. - Soit B un groupe connexe, localement connexe et admettant un groupe revêtement simplement connexe $\tilde{\mathrm{B}}$. Toute extension localement inessentielle $\mathrm{E}(\mathrm{B}, \mathrm{F})$ admet un sous-groupe connexe $\widehat{\mathrm{B}}$, groupe revêtement $\widehat{\mathrm{B}}(\mathrm{B}, \mathrm{H})$ de $\mathrm{B} . \mathrm{H}=\widehat{\mathrm{B}} \cap \mathrm{F}$ est un sous-groupe du centre de $\mathrm{E}$.

Soit $\tilde{p}$ la projection canonique de $\tilde{B}$ sur $B$ et $f$ la projection canonique de $\tilde{p}^{*} E$ sur $E(B, F)$, dont la restriction à $F$ est la transformation identique. $\tilde{p}^{*} E$ est localement inessentielle; mais $\tilde{B}$ étant simplement connexe et loca. lement connexe, tout isomorphisme local de $\tilde{B}$ dans $\tilde{p}^{*} E$ se prolonge en un isomorphisme $v ; \tilde{p}^{*} E$ est donc une extension inessentielle sur $\tilde{B} . f v(\tilde{B})$ est un sous-groupe connexe de $E$ qui se projette sur $B$ et qui est un groupe revètement $\widehat{B(B}, H)$ avec $H=\widehat{B} \cap F$. Si $\tilde{B}=\tilde{B}(B, \pi)$, on a $f v(\pi=H$; or $v(\pi)$ est dans le centre de $v(\tilde{B})$ et il permute avec chaque élément de $F$, conme on voit de $(6.1), \pi$ étant le noyean de $\tilde{p}$.

Soit $u$ un système de représentants de $B$ dans $\tilde{B}$; le cocycle $(g, \xi)$ défini par $u$ et associé à $E(B, F)$ est tel que $g$ prend ses valeurs dans $H$, c'est-àdire dans le centre $C$ de $F$. D'autre part, puisque nous pouvons supposer $u$ localement continu, si $p$ désigne la projection canonique de $E$ sur $E / C, p u$ est un système de représentants de $B$ dans $E / C$ localement continu et qui définit le cocycle (const., $p \xi$ ):

Coroluarre 1. - Dans les hypothèses de la Prop. 13.4, toute extension localement inessentielle est préinessentielle.

En remplaçant $C$ par $H$ dans la démonstration, on obtient:

Corolimarre 2. - Dans les hypothèses de la Prop. 13.4, $\mathrm{E} \mathrm{H}(\mathrm{B}, \mathrm{F} / \mathrm{H})$ est une extension inessentielle.

Proposition 13.5. - Soit B un groupe connexe, localement connexe et admettant un groupe revêtement simplement connexe: supposons de plus que toute extension de tout groupe abélien localement compact par B soit localement inessentielle (resp. inessentielle). Alors si $\mathrm{F}$ est un groupe localement compact résoluble, toute extension $\mathrm{E}(\mathrm{B}, \mathrm{F})$ est localement inessentielle (resp. inessentielle).

L'hypothèse de compacité locale a le seul but de nous assurer que la suite de composition des adhérences des sous-groupes des commutateurs de $F$ a une longeur finie ([21], thé. 5): nous utiliserons ce fait pour faire une 
démonstration par récurrence. Soit $D$ l'adhérence du sous-groupe des commutateurs de $F$ : $E / D$ est une extension du groupe abélien lòcalement compact $F / D$ par $B$. Il y a done un sous-groupe $\widehat{B}$ de $E[D$, groupe revêtement de $B$. Par l' hypothèse d'induction toute extension de $D$ par $B$ est localement inessentielle (resp. inessentielle): il en sera de même de toute extension de $D$ par $\widehat{B}$ (Lemme 12.2, Coroll.). Si $u$ est un système de représentants de $B$ dans $\widehat{B}$, et si $v$ est un système de représentants de $E / D$ dans $E$, nous pouvons supposer que la restriction de $v$ à $\widehat{B}$ est localement un isomorphisme (resp. un isomorphisme); $v u$ est donc un système de représentants de $B$ dans $E$ qui est un isomorphisme local (resp. un isomorphisme.

Le lemme suivant nous sera utile dans la suite:

LEMME 13.1. - Soit $\mathrm{B}$ un groupe abélien et $\mathrm{E}(\mathrm{B}, \mathrm{F})$ une extension $\grave{a} l a$. quelle est associé un cocycle $(\mathrm{g}, \xi)$ tel $q u$ ' il existe $\mathrm{x} \varepsilon \mathrm{B}$ avec $\xi_{\mathrm{x}}(\mathrm{y}) \neq \mathrm{y}$ pour tout $\mathrm{y} \varepsilon \mathrm{F}$. Alors $\mathrm{E}(\mathrm{B}, \mathrm{F})$ est algébriquement inessentielle. Si de plus (g. $\xi$ ) est défini par un système de représentants continu au point $\mathrm{x}_{0}, \mathrm{E}(\mathrm{B}, \mathrm{F})$ est ines. sentielle.

Soit $u$ un système de représentants définissant $(g, \xi)$, et $N$ le centralisateur de $u(x)$ : nous allons montrer que $N$ se projette $\operatorname{sur} B$ et ne rencontre $F$ qu' à l'élément neutre.

Pour $x^{\prime} \varepsilon B$ posons $h\left(x^{\prime}\right)=u\left(x^{\prime}\right) u(x) u\left(x^{\prime}\right)^{-1} u(x)^{-1}$ : c' est un éloment de $F$, car $B$ est abélien. Puisque $y^{-1} \xi_{x}(y)$ parcourt tout $F$ lorsque $y$ parcourt $F$, il y a un elément $k\left(x^{\prime}\right) \in F$, avec $\left.k\left(x^{\prime}\right)^{-1} \xi_{x} \mid k\left(x^{\prime}\right)\right\}=h\left(x^{\prime}\right)$. Alors

$$
\left.\left.\left(k\left(x^{\prime}\right) u\left(x^{\prime}\right)\right) u(x)=k\left(x^{\prime}\right) h\left(x^{\prime}\right) u(x) u\left(x^{\prime}\right)=\xi_{x} \mid k^{\prime} x^{\prime}\right)\right\} u(x) u\left(x^{\prime}\right)=u(x)\left(k\left(x^{\prime}\right) u\left(x^{\prime}\right)\right)
$$

Donc $k\left(x^{\prime}\right) u\left(x^{\prime}\right) \in N$ : puisque $x^{\prime}$ est quelconque, $N$ se projette sur $B$. Si $y \varepsilon N \cap F, \quad y^{-1} u(x) y u(x)^{-1}=y^{-1} \xi_{x}(y)=y_{0}$, ce qui est possible uniquement pour $y=y_{0}$. Donc $N$ est algébriquement isomorphe à $B$. Si de plus $u$ est continu au point $x_{0}$, puisque $u\left(x^{\prime}\right) \rightarrow h\left(x^{\prime}\right)$ et $h\left(x^{\prime}\right) \rightarrow k\left(x^{\prime}\right)$ sont des applications continues, $x^{\prime} \rightarrow k\left(x^{\prime}\right) u\left(x^{\prime}\right)$ est un isomorphisme bicontinu $\left({ }^{25}\right)$.

\section{§ 14. - L' holomorphe d'un groupe.}

La démonstration de la Prop. 13.4 ramène la connaissance des extensions localement inessentielles de $F$ par $b$ à celle des extensions inessentielles de $F$ par un groupe revêtement de $B$. Nous verrons ici que dans certains cas toutes les extensions inessentielles de $F$ sont implicitement données, lorsqu' on en connaît une particulière.

$F$ etant un groupe localement compact, munissons $\mathfrak{A}(F)$ de la topologie de la convergence compacte; la transformation identique de $\mathfrak{A}(F)$ définit

(25) Pour cette démonstration efr. la démonstration du Lemme 3.4 de [21]. 
alors une extension inessentielle de $F$ par $\mathcal{Q}\left(F^{\prime}\right)$, d'après le Coroll. 1 à la Prop. 13.2, dont la loi de composition est donnée par

$$
\left(\alpha_{1}, y_{1}\right)\left(\alpha_{2}, y_{2}\right)=\left(\alpha_{1} \alpha_{2}, y_{1} \alpha_{1}\left(y_{2}\right)\right) \quad \alpha_{i} \varepsilon \mathfrak{Q}\left(F^{\prime}\right), \quad y_{i} \varepsilon F
$$

L'extension ainsi définie est l'holomorphe $H(F)$ de $F$.

Si $E\left(B, F^{\prime}\right)$ est une extension inessentielle de $F^{\prime}$ et si $u$ est un système de représentants de $B$ dans $E$ qui est un isomorphisme et qui délinit le cocycle (const., $\xi$ ), $\xi$ est une représentation continue de $B$ dans $\mathfrak{Q}(\boldsymbol{F}):(6.1)$ montre alors que $\xi^{*} H(F)$ est équivalente à $E\left(B, F^{\prime}\right)$. Ainsi dans ce cas on peut obtenir toutes les extensions inessentielles de $\mathrm{F}$ à partir de $\mathrm{H}(\mathrm{F})$ par la méthode $d u \& 6\left({ }^{26}\right)$.

Si $F$ est un groupe de LIE connexe, $\mathfrak{A}(F)$ pent être muni d'une structure de groupe de LIE ([10], chap. $4, \S 15$ ), dont la topologie $\widetilde{\sigma}_{L}$ est plus fine de la topologie $\sigma_{c}$ de la convergence compacte: done la transformation identi. que de $\mathscr{A}(F)$, considérée comme application de $\mathscr{A}(F)$ muni de la topologie $\mathfrak{G}_{L}$ dans $\mathfrak{G}(F)$ muni de la topologie $\widetilde{G}_{C}$ est continue: (14.1) est done aussi compatible avec la topologie produit $\mathfrak{A}(F) \times F$, où $\mathfrak{A}(F)$ est muni de sa structure de groupe de LIE. Nous allons voir qu'avec cette structure $H(F)$ est un groupe de Lit.

Il est bien évident d'abord que l'analyeité de la loi de composition (14.1) et l'analycité de l'application

$$
(\alpha, y) \rightarrow \alpha(y)
$$

de $\mathfrak{A}(F) \times F$ dans $F$ sont denx propriétés équivalentes. Puisque tout automorphisme d'un groupe de LIE connexe est projection d'un automorphisme de son groupe revêtement simplement connexe ([10] chap. 4, § 15), il suffira de montrer l'analycité de (14.2) pour les groupes simplement connexes.

Supposons donc $F$ simplement connexe et soit $\mathbf{f}$ (resp. a) l'algèbre de LIE de $F$ (resp. de $\mathfrak{Q}(F)$ ): alors ([10], chap. 4, \& 15, Prop. 2) a est l'algèbre des dérivations de f. Construisons l'holomorphe $\mathbf{h}(\mathbf{f})$, e'est-à-dire l'algèbre de LIE definie sur l'espace vectoriel somme $\mathbf{a}+\mathbf{f}$ par la loi de composition

$\left[D_{1}+Y_{1}, D_{2}+Y_{2}\right]=\left[D_{1}, D_{2}\right]+D_{1} Y_{2}-D_{2} Y_{1}+\left[Y_{1}, Y_{2}\right], \quad D_{i} \varepsilon \mathbf{a}, \quad Y_{i} \varepsilon \mathbf{f}$.

On vérifie aisément $q \mathbf{u}^{\prime}$ on obtient une algèbre de LIE, qui admet $\mathbf{f}$ comme idéal tel que $\mathbf{h} / \mathbf{f}$ est isomorphe à $\mathbf{a}$ : de plus on voit que $\mathbf{h}$ admet une sous-algèbre isomorphe $\grave{a} \mathbf{a}$ et $\mathrm{s}^{\prime} y$ projetant. C'est une extension inessentielle de $\mathbf{f}$ par a (cfr. $\$ 29)$.

A $\mathbf{h} / \mathbf{f}$ correspond un groupe de Lre simplement connexe $\tilde{H}(F)$, extension inessentielle de $F$ par le groupe $\tilde{\mathfrak{Q}}(F)$, revêtement simplement connexe

${ }^{(26)}$ Cela est vrai aussi dans le cas suivant: $B$ diseret, $F$ quelconque, $Q(F)$ muni de la topologie discrète. 
de la composante connexe de l'élément neutre de $\mathfrak{Q}(F)$. A $\tilde{H}(F)$ correspond done une représentation $\tilde{\xi}$ de $\tilde{\mathfrak{d}}\left(\boldsymbol{F}^{\prime}\right)$ dans $\mathfrak{Q}\left(F^{\prime}\right)$ : $\tilde{\xi}$ est une application sur $\mathfrak{\mathfrak { l }}\left(\boldsymbol{F}^{\prime}\right)$, car l'image de $\mathbf{h}(\mathbf{f})$ par sa représentation adjointe contient a. Tout élément de $\mathfrak{A}(F)$ pouvant ainsi s'obtenir comme restriction à $F$ d'un automorphisme intérieur de $H(F)$, nous avons bien démontré les deux propositions équivalentes :

Proposition 14.1. - Si $F$ est un groupe de Lie connexe, $\mathrm{H}(\mathrm{F})$ peut être muni d'une structure de groupe de Lie.

Proposition 14.2. - Si F est un groupe de Lie connexe, l'application $(\alpha, y)-\alpha(y)$ de $\mathfrak{E}(\mathrm{F}) \times \mathrm{F}$ dans $\mathrm{F}$ est analytique, si l'on muni $\mathfrak{G}(\mathrm{F})$ de sa structure de groupe de Lie.

D'un résultat de M. Ehresmann ([15], pag. 39, note), suit alors que les topologies $\widetilde{\sigma}_{L}$ et $\widetilde{\sigma}_{C}$ sont identiques sur $\mathfrak{A}\left(F^{\prime}\right)$. Si done $B$ est un groupe de LiE et $\xi$ est une représentation de $B$ dans $\mathfrak{Q}(F)$ continue pour la topologie $\widetilde{G}_{C}, \xi$ est aussi analytique ([10], chap. 4, $\$ 13$, Prop. 1). Donc $\xi^{*} H(F)$ est un groupe de LIE et, par le Lemme 12.2, on retrouve un cas particulier d'un théorème connu (cfr. Prop. 23.1):

CoRollarRE. - Toute extension localement inessentielle d'un groupe de Lie par un groupe de Lie est aussi un groupe de Lie.

Soit $h$ une représentation croisée de $B$ dans $F$ relative à la représentation $\xi$ : posons $f(x)=\left(\varepsilon_{x}, h(x)\right)$. $f$ est une application de $B$ dans $H\left(F^{\prime}\right)$ qu' on voit être une représentation. Réciproquement à toute représentation de $B$ dans $H(F)$ correspond, par passage an quotient, une représentation de $B$ dans $\mathfrak{Q}(E)$ et, par composition avec la fonction coordonnée $H(E) \rightarrow F$, une représentation croisée de $B$ dans $E$. Si $\xi$ et $h$ sont continus, il en est de même de $f$, et réeiproquement.

Donc en particulier:

Proposition 14.3. - Toute représentation croisée continue d'un groupe de Lie $\mathrm{B}$ dans un groupe de Lie connexe $\mathrm{F}$, relative à wne représentation continue de $\mathrm{B}$ dans $\mathfrak{O}(\mathrm{F})$, est analytique.

\section{§ 15. - L'ensemble des extensions localement inessentielles.}

Nous nous proposons maintenant de déterminer l'ensemble des extensions localement inessentielles sans avoir recours anx cocycles.

Nous arriverons à la

Proposition 15.1. - Soit B un groupe connexe et localement connexe, $\mathrm{F}$ un groupe quelconque et $\chi$ une représentation de $\mathrm{B}$ dans $\mathcal{G}(\mathrm{F})$. Sup. posons l'existence d'un groupe revêtement connexe $\tilde{\mathrm{B}}(\mathrm{B}, \mathbf{K})$ de 'B et d' une extension de $\mathrm{F}$ par $\tilde{\mathrm{B}}$ de caractère $\chi \tilde{\mathrm{p}}$. Si toutes les extensions de $\mathrm{F}$ par $\tilde{\mathrm{B}}$ ce caractère $\chi \tilde{\mathrm{p}}$ sont inessentielles et équivalentes, toutes les extensions $\mathrm{E}(\mathrm{B}, \mathrm{F})$ de caractère $\chi$ sont localement inessentielles et l'ensemble de leurs classes d'équi. valence est un groupe isomorphe au groupe quotient $\operatorname{Hom}(\mathrm{K}, \mathrm{C}) / \operatorname{Hom}^{\prime}(\mathrm{K}, \mathrm{C})$. 
Ici $\tilde{p}$ désigne l'application canonique de $\tilde{B}$ sur $B, C$ le centre de $F$, $\operatorname{Hom}(K, C)$ le groupe des représentations de $K$ dans $C$ et $\operatorname{Hom}^{\prime}(K, C)$ le sous-groupe de ces représentations qui sont la restriction à $K$ d'une représentation croisé continue de $\tilde{B}$ dans $C$ relative à $\chi \tilde{p}$, e' est-it-dire d'un elément de $\tilde{S}^{1}(\tilde{B}, C, \tilde{\chi p})$.

Puisque $\tilde{p}^{*} E$ est un gr,upe revetement de $E$ (Lemme 12.2) et puisqu' il est une extension inessentielle de $F$ par $\tilde{B}, E(B, F)$ est bien localement inessentielle. La démonstration de la Prop. 13.4 montre alors qu'à $E(B, F)$ correspond une représentation de $K$ dans $C$.

Réciproquement si $\tilde{E}(\tilde{B}, F)$ est une extension inessentielle de caractère $\chi \tilde{p}$ et $h$ est une représentation de $K$ dans $C$, considérons dans $\tilde{E}$ l'ensemble des eléments $h(\gamma) v(\gamma), \gamma \varepsilon K, v$ étant un isomorphisme de $\tilde{B}$ dans $\tilde{E}$ considéré comme système de représentants : e'est un sous-groupe $N$ du centre de $\tilde{E}$. Puisque $N \cap F=\left\{y_{0}\right\}, E=\tilde{E} / N$ admet $F$ comme sous-groupe distingue fermé; par la transitivité des groupes quotients, il y a un isomorphisme bien défini de $E / F$ sur $B$. $E$ est done une extension de $F$ par $B$, qu' on voit immédiatement être de caractère $\chi$.

Il y a ainsi une application $\operatorname{de} \operatorname{Hom}(K, C)$ sur l'ensemble des extensions de $F$ par $B$ de caractère $\chi$.

Si $h$ est prolongeable en $k \varepsilon \mathscr{E}^{1}(\tilde{B}, C, \chi \tilde{p})$, on vérifie aisément que $v^{\prime}(\tilde{x})=k(\tilde{x}) v(\tilde{x}), \tilde{x} \& \tilde{B}$, est un isomorphisme de $\tilde{B}$ dans $\tilde{E}: v^{\prime}(\tilde{B})$ contient $N$ et dono son image dans $\tilde{E} / N$ est isomorphe à $B$ et rencontre $F$ seulement an point $y_{0} . \tilde{E} / N$ est donc inessentielle et équivalente à l'extension inessentielle définie par $h=$ const., car l'image de $v^{\prime}(\tilde{x})$ et celle de $v(\tilde{x})$ déterminent dans les deux extensions le mème automorphisme de $F(k(\tilde{x}) \varepsilon C)$.

Réciproquement si $E(B \quad F)=\tilde{E / N})$ est équivalente à l'extension inessen. tielle qui correspond à $h=$ const., il y a un isomorphisme $u$ de $B$ dans $E$ tel que $F \rightarrow u(x) F u(x)^{-1}$ est le même isomorphisme que $F \rightarrow v(\tilde{x}) F v(\tilde{x})^{-1}$ si $\tilde{p}(\tilde{x})=x$. L'image réciproque de $u(B)$ dans $\tilde{E}$ contient $N$, se projette sur $\tilde{B}$, rencontre $F$ seulement à l'élément neutre et est localement isomorphe à $B$; $c^{\prime}$ est done un sous-groupe de $E$ de la forme $v^{\prime}(\tilde{B})$. On peut écrire $v^{\prime}(\tilde{x})=h(\tilde{x}) v(\tilde{x})$ avec $k(\tilde{x}) \in F:$ mais puisque les automorphismes de $F$ engendrés par $v(\tilde{x})$ et $v^{\prime}(\tilde{x})$ sont le meme, il faut avoir $k(\tilde{x}) \propto C$. On vérifie alors que $k £ \mathbb{S}^{1}(\tilde{B}, C, \chi \tilde{p})$ (cfr. \& 16).

Il nous faut encore montrer que la correspondance $h \rightarrow \tilde{E} / N$ est ane représentation pour la loi de composition définie au $\$ 9$. Puisque les extensions en etude sont fibrées préinessentielles nous pouvons employer la définition de $\left(E_{1} \odot B_{z}\right)(B, F)$ comme quotient d'un sous-groupe $\bar{E}$ de $E_{1} \times E_{2}$. Si $p_{i}, i=1,2$, est la projection canonique de $\tilde{E} \operatorname{sur} E_{i}(B, F)=\tilde{E} / \tilde{N}_{i}$, considérons les extensions tranchées $E_{i}\left(B, F, u_{i}\right)$ avec $u_{i}(x) \varepsilon p_{i} v(\tilde{B})$ et, localement, $u_{i}=p_{i} v \tilde{p}^{-1}$. Soit $p$ la représentation de $\check{E}$ dans $E_{1} \times E_{z}$ définie par $p(y v(\tilde{x}))=\left(p_{1}(y v(\tilde{x})), p_{2} v(\tilde{x})\right)$. 
On voit que $p(\tilde{E})$ est contenu dans le sous-groupe $\bar{E}$ des eléments $\left(y u_{1}(x), c u_{2}(x)\right), c \varepsilon C$; et que $p(\tilde{E})$ se projette sur $E / H=\left(E_{1} \odot E_{2}\right)$, où $H$ est le sous-groupe de $E$ des éléments $\left(c, c^{-1}\right)$. De plus $p^{-1}(p(\tilde{E} \cap H)$ n' est autre que le groupe $N$ des éléments $h_{1}(\gamma) h_{2}(\gamma) v(\gamma)=\left(h_{1} h_{2}\right)(\gamma) v(\gamma)$. En effet $v(\gamma)$ est dans la même classe à droite suivant $N_{i}$ que $h_{i}\left(\gamma^{-1}\right)$ : donc $p_{i} v(\gamma)=p_{i} h_{i}(\gamma)^{-1}=$ $=h_{i}(\gamma)^{-1}$. Ainsi $p\left(h_{1}(\gamma) h_{2}(\gamma) v(\gamma)\right)=\left(h_{2}(\gamma), h_{2}(\gamma)^{-1}\right) \varepsilon H$; réciproquement si $\left(c, c^{-1}\right)=$ $=p(y v(\tilde{x}))$ on a $c^{-1}=p_{2} v(\tilde{x})$ et donc $x=\gamma$ et $c=h_{2}(\gamma) ; p_{1}(y v(\gamma))=y h_{1}(\gamma)^{-1}=$ $=c=h_{2}(\gamma)$. C' est bien dire que $y=h_{1}(\gamma) h_{2}(\gamma)$.

$\tilde{E} / N$ est ainsi isomorphe à $p(\tilde{E})_{l}^{\prime}(p(\tilde{E}) \cap H)$. Or ce dernier groupe est algébriquement isomorphe et, vu les rapports entre $u_{i}$ et $v$, isomorphe à $\bar{E} / H$. De plus l'isomorphisme qu'on obtient ainsi de $\tilde{E} / N$ sur $\left(E_{1} \odot E_{z}\right)(B, F)$ laisse fixe les éléments de $F$ et, par projection, ceux de $B:$ il est donc un isomorphisme d'equivalence d'extension.

La proposition ainsi entièrement démontrée peut se formuler, si $\tilde{B}$ est simplement connexe:

CoRollaire 1. - Soit B un groupe connexe, localement connexe et admet. tant un groupe revêtement simplement connexe $\tilde{\mathrm{B}}$ et soit $\mathrm{F}$ un groupe tel que deux extensions inessentielles de $\mathrm{F}$ par $\tilde{\mathrm{B}}$ de même caractère soient toujours équivalentes. Alors l'ensemble des classes d'équivalence des extensions localement inessentielles $\mathrm{E}(\mathrm{B}, \mathrm{F})$ de caractère $\chi$ est vide ou est un groupe isomorphe au groupe quotient $\operatorname{Hom}(\pi, \mathrm{C}) / \operatorname{Hom}^{\prime}(\pi, \mathrm{C})$.

$\pi$ désigne le groupe de Poincarí de $B$. Notons que l'ensemble en question peut être vide, car nous n'avons pas postulé l'existence d'une extension inessentielle de $F$ par $\tilde{B}$ de caractère $\chi \tilde{p}$.

Grâce à la Prop. 11.6 on a encore:

Coroluaire $2{ }^{\left({ }^{27}\right)}$. - Dans les hypothèses du Corollaire 1 supposons de plus que toute extension de $\mathrm{F}$ par $\tilde{\mathrm{B}}$ soit équivalente à l'extension triviale. Toutes les extensions $\mathrm{E}(\mathrm{B}, \mathrm{F})$ sont alors centrales et localement inessentielles et l'ensemble de leurs classes d'équivalence est un groupe isomorphe au groupe quotient $\operatorname{Hom}(\pi, \mathrm{C}) / \operatorname{Hom}^{\prime}(\pi, \mathrm{C})$.

Puisque les représentations croisées relatives à $\xi=$ const. sont les représentations, $\operatorname{Hom}^{\prime}(\pi, C)$ est ici le groupe des reprèsentations de $\pi$ dans $C$ qui sont la restriction à $\pi$ d' une représentation continue de $\tilde{B}$ dans $C$.

Rappelons que les hypothéses de ce Corollaire 2 sont vérifiées par exemple dans les deux cas suivants:

a) $B$ connexe compact de LIE, $F$ compact de LIs.

b) $B$ connexe de LIE, $F$ semi-simple de LIE.

(2i) Ce Corollaire généralise le résultát de SHapıro [31], qui concerne le cas où $F$ et $B$ sont deux groupes de Lre connexes compacts. La démonstration de la Prop. 15.1 qui figure ici est en partie une géneralisation immédiate de celle de SHapiro. 
Pour a) cfr. [8'], \$ 52. b) est une conséquence du fait que $\mathscr{G}(F)$ est un groupe diseret; toute extension $E(B, F)$ est dono centrale. Mais le centre de $F$ est discret et donc $E$ admet un sous-groupe qui est un revêtement de $B$.

Pour $F$ disoret on obtient en particulier:

CoRollatre 3. - Soit F un groupe discret, B un groupe connexe, localement connexe et admettant un groupe revêtement simplement connexe; les groupes revêtements de $\mathrm{B}$ de symbole $\mathrm{E}(\mathrm{B}, \mathrm{F})$ forment un groupe isomorphe $\grave{a} \operatorname{Hom}(\pi, \mathrm{C})$.

Ce corollaire pourrait être démontré directement: soit grâce au Lemme 12.1 et à la Prop. 12.2, soit en atilisant un résultat de la theorie des espaces fibrés.

\section{§ 16. - Systèmes de représentants conjugués.}

Soit $u$ un système de représentants de $B$ dans l'extension $E(B, F)$; si $G$ est le plus grand sous-groupe de $E$ qui se projette sur le centre de $B, G$ est distingué. Notons par $\vartheta_{x}$ l'automorphisme $G \rightarrow u(x) G u(x)^{-1}$.

Si $v$ est un autre système de représentants de $B$ dans $E$ supposons qu'on ait $v(x)=z^{-1} u(x) z$ pour un certain $z \varepsilon E$; il faut évidemment avoir $z \varepsilon G$ et donc $v(x)=z^{-1} \vartheta_{x}(z) \mid u(x)$. On en déduit:

LEMME 16.1. - L'ensemble des systèmes de représentants (resp. des systèmes de représentants continus au point $\mathrm{x}_{0}$, resp. continus au voisinage de $\mathrm{x}_{0}$ ) de $\mathrm{B}$ dans l'extension $\mathrm{E}(\mathrm{B}, \mathrm{F})$ conjugués $\dot{a}$ un système de représentants $\mathrm{u}$ donné (resp. à un système de représentants $\mathrm{u}$ donné continu au point $\mathrm{x}_{0}$, resp. continu au voisinage de $\left.\mathrm{x}_{0}\right)$ est en correspondance biunivoque avec $\mathrm{B}^{\prime}(\mathrm{B}, \mathrm{G}$, , il (resp. $\mathbf{B}^{\mathrm{t}}(\mathrm{B}, \mathrm{G}, \vartheta)$, resp. $\mathfrak{B}^{\mathrm{L}}(\mathrm{B}, \mathrm{G},-$ - $\left.)\right)$. Dans cette correspondance aux éléments de $\mathrm{B}^{\prime}(\mathrm{B}, \mathrm{F}, \xi)$ (resp. $\mathbf{B}^{1}(\mathrm{~B}, \mathrm{~F}, \xi)$, resp. $\mathfrak{B}^{1}(\mathrm{~B}, \mathrm{~F}, \xi)$ ) correspondent les systèmes conjugués à $\mathbf{u}$ par un élément de $\mathrm{F}$.

$\xi_{x}$ désigne ici l'automorphisme de $F: F \rightarrow u(x) F^{\prime} u(x)^{-1}$ ef les 1-cobords à valeurs dans $F$ relatifs a $\xi$ sont considérés comme des 1 -cobords à valeurs dans $G$ relatifs à $\vartheta$ particuliers.

$z^{-1} \hat{V}_{x}(z)$ est un élément de $F$ : si de plus nous supposons que $u$ est un isomorphisme algébrique, en écrivant $z=y u(\gamma)$ avec $\gamma$ dans le centre de $B$, on a

$$
\begin{aligned}
z^{-1} \vartheta_{x}(z) & =(y u(\gamma))^{-1} u(x) y u(\gamma) u(x)^{-1}= \\
& =u(\gamma)^{-1} y^{-1} u(\gamma) u(x) u(\gamma)^{-1} y u(\gamma) u(x)^{-1}=y^{-1} \xi_{x}\left(y^{\prime}\right):
\end{aligned}
$$

tout 1-cobord à valeurs dans $G$ relatif $a ̀$ est donc un 1-cobord à valeurs dans $F$ relatif à $\xi$.

D' autre part si $u$ et $v$ sont deux isomorphismes algébriques avec $v(x)=h(x) u(x)$, on a

$$
\begin{aligned}
v\left(x_{1} x_{2}\right) & =h\left(x_{1} x_{2}\right) u\left(x_{1} x_{2}\right)=h\left(x_{1}\right) u\left(x_{1}\right) h\left(x_{2}\right) u\left(x_{2}\right)= \\
& =h\left(x_{1}\right) \xi_{x_{1}}\left\{h\left(x_{2}\right)\right\} u\left(x_{1} x_{2}\right):
\end{aligned}
$$


on arrive ainsi à la

Proposition 16.1. - La donnée d'un système de représentants $\mathrm{u}$ de $\mathrm{B}$ dans l' extension $\mathrm{E}(\mathrm{B}, \mathrm{F})$, qui est un isomorphisme algébrique (resp. un isomorphisme), détermine une correspondance biunivoque de l'ensemble des sousgroures $\mathrm{B}_{x}$ de $-\mathrm{E}$ algébriquement isomorphes (resp. isomorphes) à $\mathrm{B}$ et $s^{\prime} y$ projetant, sur $\mathrm{Z}^{\mathrm{t}}(\mathrm{B}, \mathrm{F}, \xi)$ (resp. $\mathscr{Z}^{\mathrm{t}}(\mathrm{B}, \mathrm{F}, \xi)$ ). Dans cette correspondance $\grave{a}$ $\mathrm{B}^{\prime}(\mathrm{B}, \mathrm{F}, \xi \xi)$ (resp. à $\mathfrak{B}^{\prime}(\mathrm{B}, \mathrm{F}, \xi)$ ) correspond le sous-ensemble des $\mathrm{B}_{\alpha}$ conjugués $\grave{a} \mathrm{u}(\mathrm{B})$. Deux $\mathrm{B}_{\alpha}$ conjugués le sont aussi par un élément de $\mathrm{F}$.

Conolluatre. - Dans les hypothèses de la Prop. 16.1 pour que tous. les $\mathrm{B}_{\alpha}$ soient conjugués entre eux, il faut et il suffit que $\mathrm{Z}^{1}(\mathrm{~B}, \mathrm{~F}, \xi)=\mathrm{B}^{\mathrm{1}}(\mathrm{B}, \mathrm{F}, \xi)$ (resp. que $\mathscr{E}^{1}(\mathrm{~B}, \mathrm{~F}, \xi)=\mathfrak{B}^{4}(\mathrm{~B}, \mathrm{~F}, \xi)$ ).

IWASAWA a montré ([21], Lemme 3.8) qu'il en est ainsi si $B$ est compact et si $F=R^{n}$. MaLcev [25] l'a montré pour $B$ semi-simple de LIE, $F$ résoluble de LiE.

\section{ChapITRE. III - EXTENSIONS DE GROUPES ABELIENS}

\section{$\S 17$. Extensions abéliennes.}

Soit $(g, \xi)$ un cocycle de $B$ à valeurs dans $F$ : supposons que $B$ soit un groupe abélien et que $g$ prenne ses valeurs dans le centre de $F$.

Alors

$$
\begin{aligned}
& g\left(x_{1} x_{2}, x\right) g\left(x, x_{1} x_{2}\right)^{-1}= \\
& =g\left(x_{1}, x_{2}\right)^{-1} \xi_{x_{1}}\left|g\left(x_{2}, x\right)\right| g\left(x_{1}, x_{2} x\right) g\left(x x_{1}, x_{2}\right)^{-1} g\left(x, x_{1}\right)^{-1} \xi_{x}\left|g\left(x_{1}, x_{2}\right)\right|= \\
& =g\left(x_{1}, x_{2}\right)^{-1} \xi_{x_{2}}\left|g\left(x_{2}, x\right)\right| g\left(x_{1}, x\right) \xi_{x_{1}} \mid g\left(x, x_{2}\right)^{-1}\left\{g\left(x, x_{1}\right)^{-1} \xi_{x_{1}} \mid g\left(x_{1}, x_{2}\right)\right\}= \\
& =g\left(x_{1}, x\right) g\left(x, x_{1}\right)^{-1} \xi_{x_{1}}\left|g\left(x_{2}, x\right) g\left(x_{2} x_{2}\right)^{-1}\right| \xi_{x}\left|g\left(x_{1}, x_{2}\right)\right| g\left(x_{1}, x_{2}\right)^{-1} .
\end{aligned}
$$

Prenons en particulier $\xi=$ const. et soit $\left(g^{\prime}\right.$, const.) un cocycle équiva. lent à ( $g$, const.): calculons avec l'aide de (2.4)

$$
\begin{aligned}
& g^{\prime}\left(x_{1}, x\right) g^{\prime}\left(x, x_{1}\right)^{-1}= \\
& \left.=h\left(x_{1}\right) h(x) g\left(x_{1}, x\right) h\left(x_{1} x\right)^{-1} h\left(x x_{1}\right) g\left(x, x_{1}\right)^{-1} h x\right)^{-1} h\left(x_{1}\right)^{-1}= \\
& =g\left(x_{1}, x\right) g\left(x, x_{1}\right)^{-1} .
\end{aligned}
$$

Pour un elément $x^{\prime} \& B$ fixe posons

$$
f_{x^{\prime}}(x)=g\left(x, x^{\prime}\right) g\left(x^{\prime}, x\right)^{-1}:
$$

(17.1) montre que $f_{x^{\prime}}$ est une représentation de $B$ dans $C$ et (17.2) qu' elle ne dépend que de la classe d'équivalence de ( $g$, const.).

Leman 17.1. - La relation (17.3) definit une application $\left(\mathrm{x}^{\prime}, \mathrm{g}\right) \rightarrow \mathrm{f}_{\mathrm{x}^{\prime}}$ de $\mathrm{B} \times \mathrm{Z}^{2}(\mathrm{~B}, \mathrm{C}$, const. $)$ dans $\operatorname{Hom}(\mathrm{B}, \mathrm{C})$ qui, pour $\mathrm{x}^{\prime}$ fixe ou pour $\mathrm{g}$ fixe, est une représentation. Si $\mathrm{g}$ et $\mathrm{g}^{\prime}$ sont cohomologues, l' image de $\left(\mathrm{x}^{\prime}, \mathrm{g}\right)$ et celle de $\left(\mathrm{x}^{\prime}, \mathrm{g}^{\prime}\right)$ coïncident. 
Que $\left(x^{\prime}, g\right) \rightarrow f_{x^{\prime}}$ soit une représentation pour $g$ fixe se déduit immédiatement de (17.1). Si $x^{\prime}$ est fixe on a

$$
\begin{aligned}
& \left(g_{1} g_{2}\right)\left(x, x^{\prime}\right)\left(g_{1} g_{2}\right)\left(x^{\prime}, x\right)^{-1}= \\
& =g_{1}\left(x, x^{\prime}\right) g_{2}\left(x, x^{\prime}\right) g_{1}\left(x^{\prime}, x\right)^{-1} g_{2}\left(x^{\prime}, x\right)^{-1}= \\
& =g_{1}\left(x, x^{\prime}\right) g_{1}\left(x^{\prime}, x\right)^{-1} g_{2}\left(x, x^{\prime}\right) g_{2}\left(x^{\prime}, x\right)^{-1},
\end{aligned}
$$

d'où le Lemme.

Si $B$ et $F^{\prime}$ sont deux groupes abéliens et $E\left(B, F^{\prime}\right)$ une extension centrale, soit $u$ un système de représentants de $B$ dans $E$ et ( $g$, const.) le cocycle $q \mathfrak{u}^{\prime}$ il définit :

$$
\begin{aligned}
u(x) u\left(x^{\prime}\right) u(x)^{-1} u\left(x^{\prime}\right)^{-t} & =g\left(x, x^{\prime}\right) u\left(x x^{\prime}\right) u\left(x^{\prime} x\right)^{-1} g\left(x^{\prime}, x\right)^{-1}= \\
& =g\left(x, x^{\prime}\right) g\left(x^{\prime}, x\right)^{-1}=f_{x^{\prime}}(x) .
\end{aligned}
$$

$f_{x^{\prime}}$ ne dépend que de $x^{\prime}$ (et de $E\left(B, F^{\prime}\right)$ ) et l'on voit que son noyeau est la projection dans $B$ du centralisateur de $u\left(x^{\prime}\right)$ dans $F$. Notons alors par $M_{g}$ le noyeau de $\left(x^{\prime}, g\right)-f_{x^{\prime}}$ pour $g$ fixe: c'est le sous-groupe de $B$ des éléments $x$ tels que $g\left(x, x^{\prime}\right)=g\left(x^{\prime}, x\right)$ pour tout $x^{\prime} \varepsilon B$ :

Proposimon 17.1. - Soient $\mathrm{B}$ et $\mathrm{F}$ deux groupes abéliens et $\mathrm{E}(\mathrm{B}, \mathrm{F})$ une extension centrale à laquelle est associé le cocy le ( $\mathrm{g}$, const. $): \mathrm{M}_{\mathfrak{g}}$ est la projection dans $\mathrm{B}$ du centre de $\mathrm{E}$.

On en déduit:

Corollaire 1. - Pour que l' extension E(B, F) soit abéiienne il faut et il suffit que, pour tout $\mathrm{x} \varepsilon \mathrm{B}, \mathrm{f}_{\mathrm{x}}$ soit la représentation constante de $\mathrm{B}$ dans $\mathrm{F}$.

Corollatre 2. - Pour que l' extension $\mathrm{E}(\mathrm{B}, \mathrm{F})$ soit abélienne il faut et il suffit qu' il lui soit associé un cocycle (g, const.) avec $\mathrm{g}\left(\mathrm{x}_{1}, \mathrm{x}_{2}\right)=\mathrm{g}\left(\mathbf{x}_{2}, \mathrm{x}_{1}\right)$ pour tout $\mathrm{x}_{1}, \mathrm{x}_{2} \_\mathrm{B}$. Tout $\mathrm{g}^{\prime}$ cohomologue $\dot{a} \mathrm{~g}$ (relativement $\grave{a} \xi=$ const.) vérifie la même relation de symétrie.

Ce denxième Corollaire peut se déduire directement de (17.4).

Le noyeau $N_{x^{\prime}}$ de $\left(x^{\prime}, g\right) \rightarrow f_{x^{\prime}}$ pour $x^{\prime}$ fixe est le sous-groupe de $Z^{2}$ $\left(B, C\right.$, const.) des $g$ tels que $g\left(x^{\prime}, x\right)=g\left(x, x^{\prime}\right)$ pour tout $x \varepsilon B$. Ainsi $N=\bigcap N_{\infty}$, $x \in B$, est le sons-groupe de $Z^{2}(B, C$, const.) des cocycles $g$ symétriques.

Proposition 17.2. - Soient B et F deux groupes abéliens: l'ensemble des classes d'équivalence des extensions presque-fibrées (resp. fibrées) abéliennes $\mathrm{E}(\mathrm{B}, \mathrm{F})$ est un groupe isomorphe a $\mathrm{N} \cap \mathbf{Z}_{0}^{2}(\mathrm{~B}, \mathrm{~F}$, const. $) / \mathrm{N} \cap \mathbf{B}_{0}^{2}(\mathrm{~B}, \mathrm{~F}$, const.) (resp. à $\mathrm{N} \cap \mathscr{F}_{\mathfrak{v}}^{2}\left(\mathrm{~B}, \mathrm{~F}\right.$, const.)/ $\mathrm{N} \cap \mathfrak{B}_{\mathfrak{v}}^{2}(\mathrm{~B}, \mathrm{~F}$, const.)).

Ce groupe peut aussi se déterminer parfois à l'aide de la Prop. 15.1. En effet tout groupe connexe localement isomorphe à un groupe abélien est lui-même abélien; d'autre part toute extension inessentielle centrale d'un groupe abelien est l'extension triviale.

Proposition 17.3. - Soit B un groupe abélien connexe et localement connexe; $\mathrm{F}$ un groupe abélien et $\tilde{\mathrm{B}}(\mathrm{B}, \mathrm{K})$ un groupe revêtement connexe de $\mathrm{B}$ tel que toute extension centrale de $\mathrm{F}$ par $\tilde{\mathrm{B}}$ soit équivalente à l'extension triviale. 
Alors toute extension centrale $\mathrm{E}(\mathrm{B}, \mathrm{F})$ est abélienne et localement inessentielle; l'ensemble de leurs classes d'équivalence est un groupe isomorphe au groupe quotient $\operatorname{Hom}(\mathrm{K}, \mathrm{F}) / \operatorname{Hom}^{\prime}(\mathrm{K}, \mathrm{F})$.

CoRollaIRE - Soit B un groupe abélien connexe, localement connexe et admettant un groupe revêtement simplement connexe $\tilde{\mathrm{B}} ; \mathrm{F}$ un groupe abélien. Alors toute extension centrale localement inessentielle $\mathrm{E}(\mathrm{B}, \mathrm{F})$ est abélienne et l'ensemble de leurs classes d'équivalence est un groupe isomorphe au groupe quotient $\operatorname{Hom}(\pi, \mathrm{F}) / \operatorname{Hom}^{\prime}(\pi, \mathrm{F})$.

Pour compléter, et pour mettre en évidence un certain parallelisme formel, rappellons le résultat suivant de EilienBERG-MacLaNE:

Proposition 17.4. - ([16], thé. 10.1) - B et F étant deux groupes abéliens discrets, supposons donné un groupe abélien libre $\mathrm{L}$ et un sous-groupe $\mathrm{K}$ de $\mathrm{L}$ tels que $\mathrm{L} / \mathrm{K} \approx \mathrm{B}$. Alors l'ensemble des classes d'équivalence des extensions abélien. nes $\mathrm{E}(\mathrm{B}, \mathrm{F})$ est un groupe isomorphe au groupe quotient $\operatorname{Hom}(\mathrm{K}, \mathrm{F}) / \operatorname{Hom}^{\prime}(\mathrm{K}, \mathrm{F})$.

$\operatorname{Hom}^{\prime}(K, F)$ désigne le groupe des représentations de $K$ dans $F$ qui sont la restriction à $K$ d' une représentation de $L$ dans $F$.

\section{§ 18. - Extensions abéliennes localement compactes.}

Pour tout groupe abslien localement compact $G$ nous noterons par $\widehat{G}$ le groupe dual ([36], \& 28). Il est classique que le dual $\widehat{E}$ de $E(B, F)$ est une extension de $\widehat{B}$ par $\widehat{F}$ : nous allons voir qu'il y a correspondance biunivoque entre les classes d'équivalence des extensions $E\left(B, F^{\prime}\right)$ et celles des extensions $\widehat{E}(\widehat{F}, \widehat{B})$.

LEMME 18.1. - Soit f un isomorphisme de $\mathrm{E}(\mathrm{B}, \mathrm{F})$ sur $\mathrm{E}^{\prime}(\mathrm{B}, \mathrm{F})$; l'appli. cation duale $\widehat{\mathrm{f}}$ est un isomorphisme de $\widehat{\mathrm{E}}^{\prime}(\widehat{\mathrm{F}}, \widehat{\mathrm{B}})$ sur $\widehat{\mathrm{E}}(\widehat{\mathrm{F}}, \widehat{\mathrm{B}})$.

On sait que $\widehat{f}$ est un isomorphisme de $\widehat{E^{\prime}}$ sur $\widehat{E}$ : pour voir que c'est un isomorphisme d'extension, il suffit de montrer que $\widehat{f}(\widehat{B}) \subset \widehat{B}$. Or $\widehat{B}$ est le sous-groupe de $\widehat{E^{\prime}}$ tel que $\left\langle y^{\prime}, \widehat{x^{\prime}}\right\rangle=0$, si $y^{\prime} \varepsilon F \subset E^{\prime}, \widehat{x^{\prime}} \& \widehat{B} \subset \widehat{E^{\prime}}$ et si 0 désigne l'élément neutre de $T\left({ }^{28}\right)$. D'autre part $\widehat{B}$ est le sous-groupe de $\widehat{E}$ qui annulle $F \subset E$; comme $f(F)=F$, on a $0=\left\langle f(y), \widehat{x^{\prime}}\right\rangle=\left\langle y, \bar{f}\left(\overline{x^{\prime}}\right)\right\rangle$ et done $\widehat{f}\left(x^{\prime}\right) \notin \widehat{B}$.

Corollatre 1. - Si f est un isomorphisme d'équivalence, il en est de même de $\widehat{\mathrm{f}}$.

En effet dans ce cas, si $u$ (resp. $u^{\prime}$ ) est un système de représentants de $B$ dans $E$ (resp. dans $E^{\prime}$ ), on a $f(u(x))=h(x) u^{\prime}(x)$ et donc, puisque $h(x) \varepsilon F$ et $\left\langle F, \widehat{x^{\prime}}\right\rangle=0$,

$$
<f(u(x)), \widehat{x^{\prime}}>=\left\langle u^{\prime}(x), \widehat{x^{\prime}}\right\rangle=\left\langle u(x), \widehat{f}\left(\widehat{x^{\prime}}\right)\right\rangle .
$$

Les deux derniers membres ne dépendent ni de $u$ ni de $u^{\prime}$; par passage aux quotients on obtient $\left\langle x, \widehat{x^{\prime}}\right\rangle=\left\langle x, \widehat{f}\left(\widehat{x}^{\prime}\right)\right\rangle$, d'où $\widehat{f}\left(\widehat{x^{\prime}}\right)=\widehat{x^{\prime}}$.

(28) $T$ : groupe des réels nod. 1. 
De façon analogue on peut voir que la projection de $\widehat{f}$ est la transfor. mation identique de $\bar{F}$.

Corollatre 2. - L'ensemble des classes d'équivalence des extensions abé. liennes $\mathrm{E}(\mathrm{B}, \mathrm{F})$ est en correpondance biunivoque avec l'ensemble des classes d'équivalence des extensions abéliennes $\widehat{\mathrm{E}}(\widehat{\mathrm{F}}, \widehat{\mathrm{B}})\left({ }^{* 9}\right)$.

Puisque tout groupe abélien localement compact est un groupe produit $R^{n} \times G\left({ }^{30}\right)$, oì $G$ est un groupe admettant un sous-groupe compact onvert, on pent démontrer la

Proposirron 18.1. - Soit $B$ un groupe abélien localement compact: toute extension abélienne $\mathrm{E}\left(\mathrm{B}, \mathrm{R}^{\mathrm{n}}\right)$ est équivalente à l'extension triviale.

Supposons d'abord $B=R^{m}$ : puisqu'il n'y a pas de sous-groupes com. pacts ouverts dans $R^{p}, E=R^{n+m}$ et l'on sait que dans $R^{n+m}$ tout sousgroupe isomorphe à $R^{n}$ est facteur direct. Si $B=G$, par la même raison on voit que $\bar{E}\left(B, R^{\prime \prime}\right)$ est triviale. Dans le cas général on peut écrire $E\left(R^{m} \times G, R^{n}\right)=E\left(G, R^{n+m}\right)$ et se ramener ainsi au cas précédent.

Braconnter a démontré la

Proposimion 18.2 (5], chap. 1, \& 4, Prop. 2). - Soit B un groupe abélien discret, $\mathrm{F}$ un groupe abélien connexe el localement compact: toute extension abélienne $\mathrm{E}(\mathrm{B}, \mathrm{F})$ est équivalente à l'extension triviale.

Puisque le dual d'un groupe connexe a tons ses éléments d'ordre infini, on aura:

Corollatre. - Soit B un groupe abélien localement compact dont tous les éléments $\neq \mathrm{x}_{0}$ sont d'ordre infuni et soit $\mathrm{F}$ un groupe abélien compact; toute extension abélienne $\mathrm{E}(\mathrm{B}, \mathrm{F})$ est équivalente à l'extension triviale.

Eilenberg-Madiane ont montré la

Proposition 18.3 ([16], Coroll. 11.4). - Soit F un groupe abélien discret: pour tout groupe abélien discret $\mathrm{B}$ toute extension abélienne $\mathrm{E}(\mathrm{B}, \mathrm{F})$ èst équivalente à l'extension triviale si et seulement si les éléments de $\mathrm{F}$ sont tous de hauteur infinie $\left({ }^{31}\right)$.

Done :

CoRollatre 1. - Soit F un groupe abélien dont tous les éléments sont de hauteur infinie: pour tout groupe abélien $\mathrm{B}$ les extensions abéliennes $\mathrm{E}(\mathrm{B}, \mathrm{F})$ sont algébriquement équivalentes à l' extension triviale.

D'autre part, puisque le dual d'un groupe discret dont tous les éléments sont de hauteur infinie est un groupe compact dont les éléments sont d'ordre infini ([5], chap. $1 \& 3$, Prop. 5, Coroll.):

(29) Pour un résultat analogue voir [26], thé. 8 .

(30) $R$ : groupe additif des réols.

(3i) Un element $x$ du groupe $G$ est dit de hauteur infinie si, pour tout entier $n>0$, il existe $x^{\prime} \varepsilon G$ arec $x=n x^{\prime}$. 
Corollaire 2. - Soit B un groupe abélien compact; pour que, pour tout groupe abélien compact $\mathrm{F}$, toute extension $\mathrm{E}(\mathrm{B}, \mathrm{F})$ soit équivalente à l'extension triviale, il faut et il suffit que les éléments $\neq \mathrm{x}_{0}$ de $\mathrm{B}$ soient d'ordre infini.

A la Prop. 18.3 fait pendant la

Proposition 18.4 ([16], thé. 7.2.). - Soit B un groupe abélien discret; pour que, pour tout groupe abélien discret $\mathrm{F}$, toute extension abélienne $\mathrm{E}(\mathrm{B}, \mathrm{F})$ soit équivalente à l'extension triviale, il faut et il suffit que B soit un groupe libre.

CoRollatre 1. - Soil $\mathrm{B}$ un groupe abélien libre et $\mathrm{F}$ un groupe abélien: toute extension $\mathrm{E}(\mathrm{B}, \mathrm{F})$ est algébriquement équivalente à l'extension triviale.

Puisque un groupe abélien discret libre est isomorphe à $Z^{I}\left({ }^{32}\right)$, par passage au dual on obtient le

Corollaire 2. - Soit F un groupe abélien compact: pour que, pour tout groupe abélien compact $\mathrm{B}$, toute extension abélienne $\mathrm{E}(\mathrm{B}, \mathrm{F})$ soit équivalente d̀ l'extension triviale, il faut et il suffit que $\mathrm{F}$ soit isomorphe $\grave{a} \mathrm{~T}$.

Le dual da Corollaire 1 s'énonce :

CorollaIre 3. - Soit B un groupe abélien localement compact: toute extension abélienne $\mathrm{E}\left(\mathrm{B}, \mathrm{T}^{\mathrm{T}}\right)$ est équivalente à l'extension triviale.

En combinant ces différents résultats on peut en obtenir d'autres; nous nous bornerons à citer le suivant:

Proposimion 18.5. - Soit B un groupe abélien localement compact, G $\| n$ groupe abélien dont tous les éléments sont de hauteur infinie; toute extension abélienne $\mathrm{E}\left(\mathrm{B}, \mathrm{R}^{\mathrm{n}} \times \mathrm{T}^{\mathrm{I}} \times \mathrm{G}\right)$ est algébriquement équivalente à l'extension triviale et toute extension abélienne $\mathrm{E}\left(\mathrm{B}, \mathrm{R}^{\mathrm{n}} \times \mathrm{T}^{\mathrm{I}}\right)$ est équivalente à l'extension triviale.

Rappelons ([5], chap. $3, \S 2$, thé. 3) que tout groupe abélien localement compact et connexe est isomorphe à un sous-groupe fermé de $R^{n} \times T^{I} \times G$.

\section{$\S 19$. - Extensions de $R$ par $R^{n}$.}

Proposition 19.1. - Toute extension de $\mathrm{R}$ par $\mathrm{R}^{\mathrm{n}}$ est centrale ou inessentielle.

En effet soit $(g, \xi)$ un cocycle associé à $E\left(R^{n}, R\right)$ et supposons que ni $g$ ni $\xi$ ne soient une application constante. Puisque $\xi_{x}(y)=e^{f(x)} y,(17.1)$ peut s' ocrire

$$
\begin{aligned}
& g\left(x_{1}+x_{2}, x\right)-g\left(x, x_{1}+x_{2}\right)= \\
& =g\left(x_{1}, x\right)-g\left(x, x_{1}\right)+e^{f\left(x_{1}\right)}\left(g\left(x_{2}, x\right)-g\left(x, x_{2}\right)+\left(e^{f(x)}-1\right) g\left(x_{1}, x_{2}\right):\right.
\end{aligned}
$$

ce qui montre que $g\left(x_{1}, x_{2}\right)$ est le cobord de $h(x)=\left\{g\left(x, x^{\prime}\right)-g\left(x^{\prime}, x\right) \mid 1 /\left(e^{f\left(x^{\prime}\right)}-1\right)\right.$.

Pour $n=1$ et $\xi=$ const., $x^{\prime} \rightarrow g\left(x^{2}, x\right)-g\left(x, x^{\prime}\right)$ est une représentation, donc $g\left(x^{\prime}, x\right)-g\left(x, x^{\prime}\right)=c x x^{\prime}$, où $c$ est une constante. Mais à gauche on a une expression anti-symétrique en $x$ et $x^{\prime}$, à droite une expression symétrique: donc $c=0$. De la Prop. 18.1 suit alors:

CoRollaire. - Toute extension de $\mathrm{R}$ par $\mathrm{R}$ est inessentielle.

(32) Z: groupe des ontiers. 
Notons par $x^{1}, x^{2}, \ldots, x^{n}$ les coordonnées de $x \varepsilon R^{n}$ et posons $x^{\prime}=x-x^{n}$. Si $\left(g\right.$, const.) est un cocycle de $R^{n}$ à valeurs dans $R$ on a:

$$
\begin{aligned}
g\left(x_{1}, x_{2}\right)= & g\left(x_{1}{ }^{\prime}+x_{1}^{n}, x_{2}{ }^{\prime}+x_{2}^{n}\right)= \\
= & -g\left(x_{1}{ }^{\prime}, x_{1}^{n}\right)+g\left(x_{1}{ }^{\prime}, x_{2}{ }^{\prime}+x_{1}^{n}+x_{2}^{n}\right)+g\left(x_{1}^{n}, x_{2}{ }^{\prime}+x_{2}^{n}\right)= \\
= & -g\left(x_{1}^{\prime}, x_{1}^{n}\right)+g\left(x_{1}^{\prime}, x_{2}{ }^{\prime}\right)+g\left(x_{1}{ }^{\prime}+x_{2}{ }^{\prime}, x_{1}^{n}+x_{2}^{n}\right)-g\left(x_{2}^{\prime}, x_{1}^{n}+x_{2}^{n}\right)+ \\
& +g\left(x_{1}^{n}, x_{2}^{n}\right)+g\left(x_{1}^{n}+x_{2}^{n}, x_{2}\right)-g\left(x_{2}^{n}, x_{2}{ }^{\prime}\right) .
\end{aligned}
$$

En remplaçant $g\left(x_{1}^{n}+x_{2}^{n}, x_{2}\right)-g\left(x_{2}^{\prime}, x_{1}^{n}+x_{2}^{n}\right)$ par l'expression donnée par (17.1) et en ordonnant on obtient

$$
\begin{aligned}
g\left(x_{1}, x_{2}\right)= & -g\left(x_{1}^{\prime}, x_{1}^{n}\right)+g\left(x_{1}^{\prime}+x_{2}^{\prime}, x_{1}^{n}+x_{2}^{n}\right)-g\left(x_{2}^{\prime}, x_{2}^{n}\right)+g\left(x_{1}^{\prime}, x_{2}{ }^{\prime}\right)+ \\
& +g\left(x_{1}^{n}, x_{2}^{n}\right)+g\left(x_{1}^{n}, x_{2}\right)^{\prime}-g\left(x_{2}^{\prime}, x_{1}^{n}\right) .
\end{aligned}
$$

Les trois premiers termes sont le cobord de $h(x)=g\left(x^{\prime}, x^{n}\right)$ et d'autre part $g\left(x_{1}^{n}, x_{2}^{n}\right)$ est aussi un cobord (Corollaire): done ( $g$, const.) est continâ. ment équivalent à $\left(g^{\prime}\right.$, const.) avec

$$
g^{\prime}\left(x_{1}, x_{2}\right)=g\left(x_{1}^{n}, x_{2}{ }^{\prime}\right)-g\left(x_{2}^{\prime}, x_{1}^{n}\right)+g\left(x_{1}^{\prime}, x_{2}{ }^{\prime}\right) .
$$

Les deux premiers termes, en fixant $x_{2}{ }^{\prime}$ (resp. $x_{1}^{n}$ ), donnent une représentation continue de $R^{n}$ dans $R$, done de la forme $x \rightarrow \Sigma a_{i} x^{i}$.

D'une part il faut avoir

et d'autre part

$$
g\left(x_{1}^{n}, x_{2}^{\prime}\right)-g\left(x_{2}^{\prime}, x_{1}^{n}\right)=k\left(x_{2}^{\prime}\right) x_{1}^{n}
$$

D' où

$$
g\left(x_{1}^{n}, x_{2}{ }^{\prime}\right)-g\left(x_{2}^{\prime}, x_{1}^{n}\right)=k^{\prime}\left(x_{1}^{n}\right) \sum_{i=1}^{n-1} a^{i} x_{2}{ }^{\prime} .
$$

$$
g^{\prime}\left(x_{1}, x_{2}\right)=x_{1}^{n} \sum_{i=1}^{n-1} a_{i} x_{z}{ }^{i}+g\left(x_{1}^{\prime}, x_{2}^{\prime}\right)
$$

En répétant le mème raisonnement sur $g\left(x_{1}^{\prime}, x_{2}^{\prime}\right)$, qui est un cocycle de $R^{n-1}$, on trouve enfin que $g$ est cohomologue à un cocycle

$$
g^{\prime \prime}\left(x_{1}, x_{2}\right)=\sum_{i=2}^{n} x_{1}{ }^{i-1} \sum_{j=1}^{i} a_{i j} x_{2}{ }^{j} .
$$

On voit avant tout que $g\left(x_{1}, x_{2}\right) \neq g\left(r_{2}, x_{1}\right)$ : on retrouve ainsi que toute extension abélienne de $R$ par $R^{n}$ esté équivalente à l'extension triviale.

Deux cocycles donnés par (19.1) ne seront pas cohomologues s'ils ne sont pas identiques: en effet il faudrait que leur différence fút un cobord et done fùt symétrique en $x_{1}$ et $x_{2}$. Puisque dans. (19.1) on a $m=n(n-1) / 2$ constantes $a_{i j}$, et puisq' ici les cocycles sont continus et done $\mathfrak{H}_{0}^{2}=\mathscr{H}^{2}$ (cfr. $\S 4$ ), nous avons démontrê la

Proposition 19.2. - H $\left(\mathrm{R}^{\mathrm{n}}, \mathrm{R}\right.$, const. $)$ est algébriquement isomorphe à $\mathrm{R}^{\mathrm{m}}, \mathrm{m}=\mathrm{n}(\mathrm{n}-1) / 2$. Si $\chi \neq$ const., $\mathcal{H}^{*}\left(\mathrm{R}^{\mathrm{n}}, \mathrm{R}, \chi\right)$ est réduit à l'élément neutre. 
D' autre part on a la

Proposition 19.3. - Pour $\mathrm{n} \leq 3$ toutes les extensions centrales de $\mathrm{R}$ par $\mathrm{R}^{\mathrm{n}}$ non équivalentes $\dot{a}$ l' extension triviale sont isomorphes.

Pour le démontrer on pourrait construire directement un isomorphisme de chaque extension $E\left(R^{n}, R\right)$ sur l'une d'elles; nous suivrons une autre méthode, qui peut être utile pour d'autres problèmes.

Le cocycle $g$ donné en (19.1) étant une fonction analytique, toutes les extensions centrales $E\left(R^{n}, R\right)$ sont des groupes de LIE: nous allons en calculer les constantes de structure grâce aux. équations des translations à gauche (premier groupe des paramètres).

Dans l'espace produit $R^{n} \times R,(19.1)$ définit une loi de composition telle que $(x, y)^{-1}=(-x,-g(-x, x)-y)$ : ainsi

$$
(x, y)^{-1}(x+d x, y+d y)=(d x, d y-g(x, d x)) .
$$

Les formes de PFAFF invariantes à gauche sont done données par $\omega_{i}=d x^{i} i \leq n, \omega_{n+1}=d y-g(x, d x)$ (cfr. [8'], pag. 16).

Puisque

$$
d g(x, d x)=d\left(\sum_{i=2}^{n} x^{i} \sum_{j=1}^{i-1} a_{i j} d_{\iota^{j}}\right)=\sum_{i=2}^{n}\left(\sum_{j=1}^{i-1} a_{i j} d x^{j}\right) d x^{i},
$$

les équations de MaUrer-Cartan s'écrivent:

$$
d \omega_{i}=0 \quad i \leq n, \quad d \omega_{n+1}=\sum_{j<i} a_{i j} \omega_{j} \wedge \omega_{i}
$$

Les constantes de structure différentes de zéro seront donc $c_{j, i, n+1}=a_{i j}$, $1 \leq j<i \leq n$.

Si $E^{\prime}\left(R^{n}, R\right)$ est caractérisé par $d \omega_{i}^{\prime}=0, i \leq n, d \omega_{n+1}^{\prime}=\Sigma a_{i j}^{\prime} \omega_{j}^{\prime} \wedge \omega_{i}^{\prime}$, un isomorphisme de $E\left(R^{n}, R\right)$ sur $E^{\prime}\left(R^{n}, R\right)$ est donne par une transformation du type

$$
\omega_{i}=\Sigma a_{i j} \omega_{j}^{\prime} \quad i \leq n, \quad \omega_{n+1}=\omega_{n+1}^{\prime} .
$$

La proposition sera démontrée si l'on donne, pour $n \leq 3$, une transformation (19.3) telle que (19.2) devienne par exemple

$$
d \omega_{i}^{\prime}=0 \quad i \leq n, \quad d \omega_{n+1}^{\prime}=\omega_{1}^{\prime} \wedge \omega_{2}^{\prime} .
$$

$n=2: d \omega_{3}=a_{21} \omega_{1} \wedge \omega_{2}, a_{21} \neq 0$. La transformation est évidente.

$n=3: d \omega_{4}=a_{2}, \omega_{1} \wedge \omega_{2}+a_{31} \omega_{1} \wedge \omega_{3}+a_{32} \omega_{2} \wedge \omega_{3}$. Supposons $a_{21} \neq 0 . \mathrm{Il}$ suffit alors de poser

$$
\begin{aligned}
& \omega_{1}=-\omega_{1}^{\prime} / a_{21}+\omega_{3}^{\prime} a_{32} / a_{21} \\
& \omega_{2}=\omega_{2}^{\prime}-\omega_{3}^{\prime} a_{31} / a_{21} \\
& \omega_{3}=\omega_{3}^{\prime} .
\end{aligned}
$$

Que la proposition ne soit plus vraie pour $n \geq 4$, montre l'exemple suivant. Soit $E\left(R^{4}, R\right)$ donné pas $g\left(x_{1}, x_{2}\right)=x_{1}^{2} x_{2}^{1}$ et $E^{\prime}\left(R^{4}, R\right)$ donné par 
$g^{\prime}\left(x_{1}, x_{2}\right)=x_{1}^{2} x_{2}^{1}+x_{1}^{3} x_{2}^{2}$. Les systèmes (19.2) correspondants sont respectivement

$$
\begin{array}{lll}
d \omega_{i}=0 & i \leq 4, & d \omega_{5}=\omega_{1} \wedge \omega_{2} \\
d \omega_{i}=0 & i \leq 4, & d \omega_{5}=\omega_{1} \wedge \omega_{2}+\omega_{2} \wedge \omega_{3} .
\end{array}
$$

On peut alors montrer par un calcul qu'il n'y a pas de transformations (19.3) possibles $\left({ }^{3 *}\right)$.

Proposimion 19.4. - Toutes les extensions inessentielles $\mathrm{E}\left(\mathrm{R}^{\mathrm{n}}, \mathrm{R}\right)$ non équivalentes à l'extension triviale sont isomorphes.

En effet si $u$ (resp. $u^{\prime}$ ) est un système de représentants de $R^{\prime \prime}$ dans $E$ (resp. $E^{\prime}$ ) qui est un isomorphisme et qui définit le cocyole (const., $e^{\Sigma r_{i} x^{i}}$ ) (resp. (const., $\left.e^{x^{l}}\right)$ ), la transformation $y u(x) \rightarrow y u^{\prime}(\bar{x})$ avec $\bar{x}^{i}=\Sigma r_{i} x^{i}, \bar{x}^{l}=x^{i}$, $i>1$ est un isomorphisme (supposant $r_{1} \neq 0$ ).

\section{§ 20. - Les extensions de $R^{n}$ par $R$.}

Proposition 20.1. - Toute extension de $\mathrm{R}^{\mathrm{n}}$ par $\mathrm{R}$ est inessentielle.

Le cas $n=1$ étant résolu dans le Coroll. à la Prop. 19.1, la démonstra. tion sera faite par récurrence. Soit $(g, \xi)$ un cocycle associé à $E\left(R, R^{\prime \prime}\right)$ et soit $R^{s}$ le sous-groupe de $R^{n}$ des éléments tels que $\xi_{x}(y)=y$ pour tout $x \varepsilon R$. $R^{s}$ est distingué dans $E$ (Lemme 7.1).

Supposons $0<s<n: E / R^{s}\left(R, R^{n} / R^{s}\right)$ est, par l'hypothèse d'induction, nne extension inessentielle: soit $u$ un isomorphisme de $R$ dans $E / R^{s}$ et $p$ la projection canonique de $E$ sur $E / R^{s} \cdot p^{-\mathrm{t}} u(R)$ est alors une extension de $R^{s}$ par $R$ à laquelle on peut anssi appliquer l'hypothèse d'induction. Il y a ainsi un système de représentants $v$ de $E / R^{s}$ dans $E$ dont la restriction à $u(R)$ est un isomorphisme: $v u$ est alors bien un isomorphisme de $R$ dans $E$.

Si $s=n$, l'extension est centrale et l'on peut refaire le même raisonnement en prenant un $R^{p} \subset R^{n}, 0<p<n$, quelconque. Si $s=0$ on est dans les hypothèses du Lemme 13.1, qui achève la démonstration.

Corollaire 1. - Pour tout $\chi, \mathscr{H}^{2}\left(\mathrm{R}, \mathrm{R}^{\mathrm{n}}, \chi\right)$ est réduit à l'élément neutre.

D'autre part le Coroll. à la Prop. 5.1 et le $\$ 13$ donnent:

CoRoluatre 2. - Il y a une correspondance biunivoque entre les classes d'extensions $\mathrm{E}\left(\mathrm{R}, \mathrm{R}^{\mathrm{n}}\right)$ isomorphes et les classes de sous-groupes conjugués $\grave{a}$ une dimension de $\mathrm{GL}(\mathrm{n}, \mathrm{R})$. Pour $\mathrm{n} \geq 2$ le nombre de ces classes est infini.

En effet deux extensions sont isomorphes s'il y a une constante réelle $a \neq 0$ et un $\varphi \varepsilon \mathfrak{Q}\left(R^{n}\right)=G L(n, R)$ tels que $\chi_{a x}^{\prime} \varphi={ }^{\prime} \chi_{x}$, si $\chi$ et $\chi^{\prime}$ sont les caractères des deux extensions.

La dernière partie du Corollaire résulte du fait que dans $G L(n, R)$ avec

${ }^{(33)}$ On peut aussi montrer très facilement que les sous-groupes des commutateurs de $E$ et de $E^{\prime}$ ne sont pas isomorphes. 
$n \geq 2$ les sous-groupes décrits par les matrices

$$
\left(\begin{array}{cccc}
e^{x} & & & \\
& e^{r x} & & \\
& 1 & & \\
& & \ddots & \\
& & & 1
\end{array}\right) \quad, \quad\left(\begin{array}{llll}
e^{x} & & & \\
& e^{r x} & & \\
& & 1 & \\
& & \ddots & \\
& & & 1
\end{array}\right)
$$

avec $r \neq r^{\prime}$ ne sont pas conjugués.

La recherche des classes de sous-groupes conjugués à une dimension de $G L(n, R)$ est facilitée par la

Proposition 20.2. - Soit $\sigma(\mathrm{t}),-\infty \leq \mathrm{t} \leq \infty$, un sous-groupe à une dimension de $\mathrm{GL}(\mathrm{n}, \mathrm{R})$; il existe une matrice $\alpha \varepsilon \mathrm{GL}(\mathbf{n}, \mathbf{R})$ telle que, pour tout $\mathrm{t}, \alpha \sigma(\mathrm{t}) \alpha^{-1}=\sigma^{\prime}(\mathrm{t}) \sigma^{\prime \prime}(\mathrm{t})$ oì $\sigma^{\prime}(\mathrm{t})$ est une matrice diagonale ou conjuguée dans $\mathrm{GL}(\mathrm{n}, \mathrm{C})$ à une matrice diagonale, et $\sigma^{\prime \prime}(\mathrm{t})$ est une matrice triangulaire dont les éléments $\mathrm{a}_{\mathrm{ij}}$ sont des polynomes : $\mathrm{a}_{\mathrm{ij}}=0 \mathrm{i}<\mathrm{j}, \mathrm{a}_{\mathrm{ii}}=1, \mathrm{a}_{\mathrm{ij}}=\sum_{\mathrm{k}=1}^{i-\mathrm{j}} c_{\mathrm{k}}^{\mathrm{ij}} t^{\mathrm{k}}$. Les $\mathrm{c}_{\mathrm{k}}^{\mathrm{ij}}$ avec $\mathrm{k}>1$ sont déterminés par les $\mathrm{c}_{1}^{\mathrm{ij}}$ et ces derniers, pour un choix convenable de $\alpha$, sont tels que $\mathrm{c}_{1}^{\mathrm{i}, \mathrm{i}-1}=0,1$ et $\mathrm{c}_{1}^{\mathrm{ij}}=0$ si $\mathrm{i}-\mathrm{j}>1$.

Pour ce choix de $\alpha, \sigma^{\prime}(t)$ et $\sigma^{\prime \prime}(t)$ prennent le nom de facteur diagonal et respectivement facteur triangulaire de $\sigma(t)$.

Considérons $\sigma(t)$ comme élément de $G L(n, C), C$ étant le corps des nom. bres complexes; il existe une matrice $\beta$ telle que $\beta \sigma(t) \beta^{-1}=\sigma_{1}(t) \sigma_{2}(t)$, avec $\sigma_{1}(t)$ diagonale et. $\sigma_{2}(t)$ triangulaire avec les éléments de la diagonale principale égaux à 1 ([23], \& 3). Soient $b_{i j}(t)$ les éléments de $\sigma_{2}(t)$, avec $b_{i j}=0$ si $i<j, b_{i i}=1$

Puisque $\sigma_{2}(t) \sigma_{2}\left(t^{\prime}\right)=\sigma_{2}\left(t+t^{\prime}\right)$ on a

$$
\sum_{j \leq h \leq i} b_{i h}(t) b_{h j}\left(t^{\prime}\right)=b_{i j}\left(t+t^{\prime}\right):
$$

par récurrence on trouve alors

$$
b_{i j}(t)=\sum_{k=1}^{i-j} d_{k}^{i j} t^{k}, \quad d_{k}^{i j} \varepsilon C
$$

En effet cela est vrai pour $b_{i, i-1}(t)$ comme on voit immédiatement de (20.1), et si cela est supposé vrai pour $b_{i j}(t)$ avec $i-j \leq r$ on a

$$
\begin{gathered}
b_{i+1, j}\left(t+t^{\prime}\right)=\sum_{j \leq h \leq i+1} b_{i+1, h}(t) b_{h j}\left(t^{\prime}\right)= \\
=\sum_{j<h<i+1}\left(\sum_{k=1}^{i+1-h} d_{k}^{i+1, h} t^{k}\right)\left(\sum_{k=1}^{h-j} d_{k}^{h j} t^{\prime k}\right)+b_{i+1, j}(t)+b_{i+1, j}\left(t^{\prime}\right) .
\end{gathered}
$$

Or, puisque les $b_{i j}(t)$ sont des fonctions analytiques, on peut calculer la limite, pour $t^{\prime} \rightarrow 0$, de

$$
\frac{b_{i+1, j}\left(t+t^{\prime}\right)-b_{i+1, j}(t)}{t^{\prime}}=\sum_{j<h<i+1}\left(\sum_{k=1}^{i+1-h} d_{k}^{i+1, h} t^{k}\right)\left(\sum_{k=1}^{h-j} d_{k}^{h j} t^{\prime k-1}\right)+\frac{b_{i+1, j}\left(t^{\prime}\right)}{t^{\prime}}
$$

qui donne, par une simple intégration, la formule (20.2) pour $b_{i+1, j}(t)$. 
D' une façon analogue on peut la démontrer pour $b_{i, j-1}(t)$, et donc pour tout $b_{i j}(t)$, quelle que soit la valeur de $i-j$.

Mettant (20.2) dans (20-1) nous allons montrex qu' on a

$$
d_{k}^{i j}=\frac{1}{k !} \Sigma d_{1}^{i \lambda_{1}} d_{1}^{\lambda_{1} \lambda_{2}} \ldots d_{1}^{\lambda_{k-1}, j}, \quad i>\lambda_{1}>\lambda_{2}>\ldots>\lambda_{k-1}>j
$$

En effet (20.1) devient

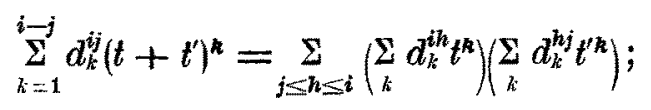

puisque (20.3) est vrai pour $k=1$, faisons un raisonnement par récurrence. En comparant dans (20.4) les coefficients de $t^{l} t^{\prime k-l}$ pour $0<l<k$, on a

$$
\left(\begin{array}{l}
k \\
l
\end{array}\right) d_{l}^{i j}=\sum_{j<h<i} d_{l}^{i h} d_{k-l}^{h j}
$$

Puisque $l$ et $k-l$ sont plus petits que $k$, par induction on aura

$$
\begin{aligned}
& \left(\begin{array}{l}
k \\
l
\end{array}\right) d_{k}^{i j}=\sum_{j<h<i}\left(\frac{1}{l !} \underset{h<\ldots \lambda_{2}<\ldots<i}{\Sigma} d_{1}^{i \lambda_{1}} d_{1}^{\lambda_{1} \lambda_{2}} \ldots d_{1}^{\lambda_{l}-1} h\right)\left(\frac{1}{(k-l) !} \underset{j<\ldots \mu_{i}<\ldots h}{\Sigma} d_{1}^{h_{1} \mu_{1}} d_{1}^{\mu_{1} \mu_{2}} \ldots d_{1}^{\mu_{k-l-1}}\right)= \\
& =\frac{1}{l !(k-l) !} \Sigma d_{1}^{i \lambda_{1}} d_{1}^{\lambda_{1} \lambda_{2}} \ldots d_{1}^{\lambda_{l}-1^{h}} d_{1}^{h \mu_{1}} \ldots d_{1}^{\mu_{k-l-1}}, \quad i>\ldots>\lambda_{i}>\ldots>h>\ldots \mu_{i} \ldots>j
\end{aligned}
$$

d'où la formule (20.3).

LEMme 20.1. - Soient $\sigma(\mathrm{t})$ et $\tau(\mathrm{t}),-\infty \leq \mathrm{t} \leq \infty$, deux sous-groupes $\dot{a}$ une dimension de $\mathrm{GL}(\mathrm{n}, \mathrm{C})$, les éléments des matrices $\sigma(\mathrm{t})$ et $\tau(\mathrm{t})$ étant respectivement $\Sigma_{\mathrm{k}} \mathrm{d}_{\mathrm{k}}^{\mathrm{ij}} \mathrm{t}^{\mathrm{k}}+\delta_{\mathrm{ij}}$ et $\sum_{\mathrm{k}} \mathrm{c}_{\mathrm{k}}^{\mathrm{ij}} \mathrm{t}^{\mathrm{k}}+\delta_{\mathrm{ij}}$. Pour que les deux sous-groupes soient conjugués il faut et il suffit qu' il existe $\gamma=\left(\gamma_{\mathrm{ij}}\right) \& \mathrm{GL}(\mathrm{n}, \mathrm{C})$ avec

$$
\underset{j<h}{\Sigma} \gamma_{i h} d_{1}^{h j}=\underset{h<i}{\Sigma} c_{1}^{i h} \gamma_{h j}
$$

Nécessité. Soit $\gamma \sigma(t)=\tau(t) \gamma$. Alors

$$
\underset{i<h}{\sum} \gamma_{i n} \underset{k}{\Sigma} d_{k}^{h j} t^{h}+\gamma_{i j}=\underset{h<i}{\sum} \sum_{k} c_{k}^{i h} t^{h} \gamma_{h j}+\gamma_{i j}
$$

d'où

$$
\underset{k+j \leq h}{\sum} \gamma_{i h} d_{k}^{h j}=\sum_{h \leq i-k} c_{k}^{i h} \gamma_{h j}
$$

en particulier pour $k=1$.

Suffisance. Montrons que la condition du Lemme est suffisante pour obtenir (20.5), qui est évidemment équivalent à $\gamma \sigma=\tau \gamma$. Nous ferons usage 
de (20.3) appliqué aux $d_{k}^{i j}$ et aux $c_{k}^{i j}$.

$$
\begin{aligned}
\sum_{k+j \leq h} \gamma_{i h} d_{k}^{h j} & =\sum_{k+j \leq h} \gamma_{i h} \frac{1}{k !} \Sigma d_{1}^{h \lambda_{1}} d_{1}^{\lambda_{1} \lambda_{3}} \ldots d_{1}^{\lambda_{k-1} j}= \\
& =\frac{1}{k !} \sum_{h>\ldots>\lambda_{i}>\ldots j} \gamma_{i h} d_{1}^{h_{1}} d_{1}^{\lambda_{1} \lambda_{2}} \ldots d_{1}^{\lambda_{k-1} j}= \\
& =\frac{1}{k !} \Sigma c_{1}^{i_{\mu_{1}}} \gamma_{\mu_{1} \lambda_{2}} d_{1}^{\lambda_{1} \lambda_{2}} \ldots d_{1}^{\lambda_{k-1} j}=\ldots= \\
& =\frac{1}{k !} \Sigma c_{1}^{i \mu_{1}} c_{1}^{\mu_{1} \mu_{2}} \ldots c_{1}^{\mu_{k-1}-\mu_{k}} \gamma_{\mu_{k} j}=\sum_{\mu_{k} \leq i-k}^{\Sigma} c_{k}^{i \mu_{k}} \gamma_{\mu_{k} j} .
\end{aligned}
$$

LFMnge 20.2. - La matrice $\sigma(\mathrm{t})$ dont les éléments sont $\sum_{\mathrm{k}=1}^{\mathrm{i}-j} \mathrm{~d}_{\mathrm{k}}^{\mathrm{jj}} \mathrm{t}^{\mathrm{k}}+\delta_{\mathrm{ij}}$ est, pour tout $\mathrm{t}$, conjuguée à la matrice réelle $\tau(\mathrm{t})$ dont les éléments sont $\sum_{\mathrm{k}=1}^{\mathrm{i}-\mathrm{j}} \mathrm{c}_{\mathrm{k}}^{\mathrm{ij}} \mathrm{t}^{\mathrm{k}}+\delta_{\mathrm{j}}$ avec $\mathrm{c}_{1}^{\mathrm{i}, \mathrm{i}-1}=1$ si $\mathrm{d}_{1}^{\mathrm{i}, \mathrm{i}-1} \neq 0, \mathrm{e}_{\mathrm{i}}^{\mathrm{i}, \mathrm{i}-1}=0$ si $\mathrm{d}_{1}^{\mathrm{i}, \mathrm{i}-1}=0$ et $\mathrm{e}_{1}^{\mathrm{ij}}=0$ pour $\mathrm{i}-\mathrm{j}>1$.

Nous montrerons l'existence d'une matrice $\gamma$ avec $\gamma_{i j}=0$ pour $i<j$ et telle que $\gamma \sigma=\tau \gamma$. La condition du Lemme 20.1 s'écrit

$$
\underset{j<h \leq i}{\Sigma} \gamma_{i h} d_{1}^{h j}=c_{1}^{i, i-1} \gamma_{i-1, j}
$$

plus en détail

$$
\begin{array}{ll}
(j=1, i=2) & \gamma_{22} d_{1}^{24}=c_{1}^{24} \gamma_{11} \\
(j=1, i=3) & \gamma_{32} d_{1}^{24}+\gamma_{33} d_{1}^{31}=c_{1}^{32} \gamma_{21} \\
(j=1, i=4) & \gamma_{42} d_{1}^{21}+\gamma_{43} d_{1}^{31}+\gamma_{44} d_{1}^{41}=c_{1}^{43} \gamma_{31} \\
\ldots \ldots \ldots \ldots & \ldots \ldots \ldots \ldots \\
(j=2, i=3) & \gamma_{33} d_{1}^{32}=c_{1}^{32} \gamma_{22} \\
(j=2, i=4) & \gamma_{43} d_{1}^{32}+\gamma_{44} d_{1}^{42}=c_{1}^{43} \gamma_{32} \\
\ldots \ldots \ldots & \ldots \ldots
\end{array}
$$

Posons $\gamma_{i 1}=1, i=1,2, \ldots, n$. Les équations $(i-1, i)$ pour $i \geq 2$ déterminent alors les $\gamma_{i i}: \gamma_{i i}=\gamma_{i-1}, i-1 / d_{1}^{i, i-1}$ si $d_{1}^{i, i-1} \neq 0, \gamma_{i i}=\gamma_{i-1}, i-1$ si $d_{1}^{i, i-1}=0$. Les équations $(i-2, i), i \geq 3$, permettent de déterminer les $\gamma_{i-1, i}$ : si le coefficient de $\gamma_{i-1, i}$ est nul, nous poserons $\gamma_{i-1, i}=1$. On voit ainsi que d'une façon générale, les équations $(i-k-1, i)$ pour $i \geq k+2$ déterminent les $\gamma_{i-k, i}$ lorsqu' on connait les $\gamma_{i j}$ avec $i-j<k$. On peut ainsi déterminer par récurrence tous les $\gamma_{i j}$; en particulier notons que $\gamma_{i i} \neq 0$. $\sigma(t)$ et $\tau(t)$ sont bien conjugués dans $G L(n, C)$. Que $\tau(t)$ soit réelle suit de $(20.3)$.

Si $\sigma(t)$ est elle-même réelle, les $\gamma_{i j}$ sont réels et donc les deux matrices sont conjuguees dans $G L(n, R)$. 
Revenons à la Prop. 20.2: nous avons montré que $\sigma_{2}(t)$ est conjugué par $\gamma$ à une matrice réelle $\tau(t):$ done

$$
\sigma(t)=\left(\beta^{-1} \sigma_{1}(t) \beta\right)\left(\beta^{-1} \gamma^{-1} \tau(t) \gamma \beta\right) \text {. }
$$

Nous allons voir que $\beta^{-1} \sigma_{1}(t) \beta$ est une matrice réelle: il en suivra que $\beta^{-1} \gamma^{-1} \tau(t) \gamma \beta$ est aussi réelle, car le produit $\sigma(t)$ l'est. Il existera donc une matrice réelle $\alpha$ telle que $\alpha\left(\beta^{-1} \gamma^{-1} \tau(t) \gamma^{\beta}\right) \alpha^{-1}=\tau(t) ;$ posons $\sigma^{\prime}(t)=\alpha \beta^{-1} \sigma_{1}(t) \alpha^{-1}$ et $\sigma^{\prime \prime}(t)=\tau(t)$. Alors $\sigma(t)=\alpha^{-1} \sigma^{\prime}(t) \sigma^{\prime \prime}(t) \alpha$ comme la Prop. 20.2 affirme.

Pour montrer que $\beta^{-t} \sigma_{i}(t) \beta$ est réelle, calculons les éléments $s_{i j}$ de $\sigma(t)$ : si $\beta_{i j}$ sont les éléments de $\beta, \beta_{i j}^{*}$ ceux de $\beta^{-1}$ et $e^{r_{i} t}$ ceux de $\sigma_{1}(t)$, nous avons:

$$
\begin{aligned}
s_{i j} & =\sum_{l \leq h} \beta_{i h}^{*} e^{r_{h} t b_{h l} \beta_{l j}}= \\
& =\sum_{l<h}^{\sum} \beta_{i h}^{*} e^{r_{h} t}\left(\sum_{k}^{\Sigma} d_{k}^{h l} t^{n}\right) \beta_{l j}+\sum_{h} \beta_{i h}^{*} e^{r_{h} \beta_{h j}} .
\end{aligned}
$$

$s_{i j}$ étant réel, le coefficient de chaque puissance de $t$ doit aussi l'être: en particulier done $\sum_{h} \beta_{i h}^{*} e^{r_{h}} \beta_{h j}$ : ce qui exprime bien que $\beta^{-1} \sigma_{1}(t) \beta$ est réel. La Prop. 20.2 est ainsi entièrement démontrée.

\section{§ 21. - Sur les extensions de $K^{n} \times T^{s}$ par $K^{m} \times T^{r}$.}

Lemme 21.1 - A toute extension centrale de $\mathrm{R}^{\mathrm{n}} \times \mathrm{T}^{\mathrm{s}}$ par $\mathrm{R}^{\mathrm{m}}$ est associé un cocycle (g, const.) où $\mathrm{g}_{\mathrm{i}}$ est dcnné par la formule (19.1), éventuellement modulo 1 .

Nous considérons $R^{n} \times T^{s}$ comme le groupe produit $k_{k_{1}} \times R_{2} \times \ldots \times R_{n} \times$ $\times T_{1} \times T_{2} \times \ldots \times T_{s}$ où chaque $R_{i}\left(\right.$ resp. $\left.T_{i}\right)$ est isomorphe à $R$ (resp. à $\left.T\right)$ : $g_{i}$ est alors la $i$-ième coordonnée de $g$. La démonstration est triviale, les calculs faits au $\& 19$ restant valables, modulo 1 , pour les cocycles à valeurs dans $T$.

Rappelons que toute extension de $T^{s}$ est centrale (Prop. 11.4).

Corollaire 1. - Toute extension centrale de $\mathrm{R}^{\mathrm{n}} \times \mathrm{T}^{\mathrm{s}}$ par $\mathrm{T}^{\mathrm{r}}$ est équivalente à l'extension triviale.

En effet soit $E\left(T^{r}, R^{n} \times T^{s}\right)$ une telle extension et $\tilde{p}$ la projection cano. nique de $R^{r}$ sur $T^{r}$ : à $\tilde{p}^{*} E$ est associé un cocycle $(g$, const.) avec $g$ périodique; vu le Lemme, $g$ est done un cobord. C'est dire que $\tilde{p}^{*} E$ et $E$ sont abéliens. On applique alors la Prop. 18.5.

En particulier done

Corolilatre 2. - Toute extension de $\mathrm{T}^{\mathrm{s}}$ par $\mathrm{Tr}$ est équivalente à l' exten. sion triviale.

Ce dérnier résultat montre $q u^{\prime}$ il $y$ a des espaces fibrés dont la fibre et la base sont des groupes topologiques, et dont le groupe structural est le groupe des translations à droite de la fibre, qui n'admettent ancune structure 
d'extension. Il y a en effet des espaces fibres de fibre $T^{s}$ et de base $T^{r}$ qui ne sont pas triviaux, même si le groupe structural est lui-même $T^{s}$.

Proposition $21.1-A$ toute extension centrale $\mathrm{E}\left(\mathrm{R}^{\mathrm{m}} \times \mathrm{T}^{\mathrm{r}}, \mathrm{R}^{\mathrm{n}} \times \mathrm{T}^{\mathrm{s}}\right)$ est associé un cocycle ( $\mathrm{g}$, const.) oì $\mathrm{g}$ est un cocycle de $\mathrm{R}^{\mathrm{m}}$ et les $\mathrm{g}_{\mathrm{i}}$ sont donnés par la formule (19.1), éventuellement modulo 1.

En effet le Coroll. 1 permet d'affirmer que $E$ a aussi une structure de symbole $E\left(R^{m}, T^{r} \times R^{n} \times T^{s}\right)$, à laqũelle on peut appliquer le Lemme.

CoRollatre. $-\mathscr{H}^{2}\left(\mathrm{R}^{\mathrm{m}} \times \mathrm{T}^{\mathrm{r}}, \mathrm{R}^{\mathrm{n}} \times \mathrm{T}^{\mathrm{s}}\right.$, const. $)$ est indépendant de $\mathrm{r}$ et algébriquement isomorphe à $\mathrm{R}^{\mathrm{q}}$ où $\mathrm{q}=(\mathrm{n}+\mathrm{s})(\mathrm{m}-1) \mathrm{m} / 2$.

Proposition 21.2. - Toute extension de $\mathrm{R}^{\mathrm{n}} \times \mathrm{T}^{\mathrm{s}}$ par $\mathrm{R}$ est inessentielle. $E\left(R, R^{n} \times T^{s}\right)$ a en effet anssi une structure de symbole $E\left(E^{\prime}\left(R, R^{n}\right), T^{s}\right)$, car $T^{s}$ est caractéristique dans $R^{n} \times T^{s} . E^{\prime}\left(R, R^{n}\right)$ est inessentielle: un sous-groupe de $E$ est done une extension de $T^{s}$ par $R$ : qu'elle soit inessentielle suit, par un raisonnement analogue à celui fait pour démontrer la Prop. 20.1, du

LEMnce 21.2. - Toute extension de $\mathrm{T}$ par $\mathrm{R}$ est équivalente à l'extension triviale.

Toute extension $E(R, T)$ étant centrale, ce Lemme se démontre comme le Coroll. à la Prop. 19.1.

La Prop. 21.2 admet, en s'appuyant sur le Lemme 12.2 et le Coroll. 2 à la Prop. 15.1, le

Corollaire. - Toute extension de $\mathrm{R}^{\mathrm{n}} \times \mathrm{T}^{\mathrm{s}}$ par $\mathrm{T}$ est inessentielle.

\section{Chapitre IV. - APPLications AUX gROUPES DE LIE.}

\section{§ 22. - Les groupes résolubles.}

Proposition 22.1. - Soit B un groupe de Lie connexe tel que toute extension d'un groupe de Lie abélien par B soit localement inessentielle. Alors pour tout groupe de Lie $\mathrm{F}$, toute extension $\mathrm{E}(\mathrm{B}, \mathrm{F})$ est localement inessentielle.

Désignons par $F^{\prime}$ le plus grand sous-groupe résoluble distingué connexe de $F$ : il est distingué dans $E$, car il est caractéristique dans $F$. $F / F^{\prime}$ étant semi-simple, $E / F^{\prime}\left(B, F / F^{\prime}\right)$ est localement inessentielle (cfr. $\$ 15$ ) et contient donc un sous-groupe $\bar{B}$, groupe revêtement de $B$. Le plus grand sous-groupe de $E$ se projetant sur $\widehat{B}$ est une extension $E^{\prime}\left(\widehat{B}, F^{\prime}\right)$ qui est localement inessentielle (Prop. 13.5)- D' où la proposition.

$\mathrm{Vu}$ la Prop. 21.2 et son Corollaire, on peut formuler :

Corollandre 1. - Soit $\mathrm{F}$ un groupe de Lie: toute extension $\mathrm{E}(\mathrm{B}, \mathrm{F})$ est inessentielle (resp. localement inessentielle) si $\mathrm{B}$ est isomorphe $\dot{a} \mathrm{R}$ (resp. $\grave{a} \mathrm{~T}$ ).

D'autre part tout groupe de Lre résoluble admet $R$ on $T$ comme groupe quotient :

CoRoulatre 2. - Tout groupe de Lie résoluble connexe admet une structure d'extension inessentielle $\mathrm{E}(\mathrm{B}, \mathrm{F})$, où $\mathrm{B}$ est isomorphe $\grave{a} \mathrm{R}$ ou $\grave{a} \mathrm{~T}$. 
Par induction sur la dimension on pent déduire de ce Corollaire plasieurs résultats bien connus sur les groupes de LiE résolubles. En particulier la proposition suivante, contenue dans un théorème de Chevallegr [9] (ofr. aussi Prop. 23.1):

Proposition 22.2. - B et $\mathrm{F}$ étant deux groupes de Lie résolubles, toute extension $\mathrm{E}(\mathrm{B}, \mathrm{F})$ est un groupe de Lie résoluble.

Il suffit de le montrer pour $B$ et $F$ connexes. Si $n>1$ est la dimension de $B$ et si la proposition est vraie pour $m<n$, considérons la structure d'extension de $E$ de symbole $E\left(B / B^{\prime}, E^{\prime}\left(B^{\prime}, F^{\prime}\right)\right)$, où $B^{\prime}$ est un sous-groupe distingué de $B$ de dimension $<n$. Par l'hypothèse d'induction $E^{\prime}$ est un groupe de LIE, et $E$ donc aussi.

Si $n=1, B$ est isomorphe à $R$ ou à $T$; le Coroll. 2 ci-dessus et le Coroll. à la Prop. 14.2 achèvent alors la démonstration.

\section{§ 23. - Extensions de Lie.}

Imasawa ([21], thê. 7) et Gleason ([19], thé. 1) ont démontré séparément la suivante

Proposition 231 . - Si B et F sont deux groupes de Lie, toute extension $\mathrm{E}(\mathrm{B}, \mathrm{F})$ est aussi un groupe de Lie.

On peut en tirer le

Corollatre 1. - Soient $\mathrm{B}$ et $\mathrm{F}$ deux groupes de Lie, dont $\mathrm{B}$ semi-simple; toute extension $\mathrm{E}(\mathrm{B}, \mathrm{F})$ est localement inessentielle.

Ce n'est que le théorème de Levi complété par la Proposition précédente. Gràce à la Prop. 22.1, le Coroll. 1 est évidemment équivalent au

CoRollalRE 2. - Soient $\mathrm{B}$ et $\mathrm{F}$ deux groupes de Lie dont $\mathrm{B}$ semi-simple et simplement connexe et $\mathrm{F}$ abélien: alors pour toute représentation continue $\chi$ de $\mathrm{B}$ dans $\mathfrak{G}(\mathrm{F})=\mathfrak{G}(\mathrm{F})$ muni de la topologie de la convergence compacte $\mathscr{H}^{\mathfrak{2}}(\mathrm{B}, \mathrm{F}, \chi)$ est réduit à l'élément neutre.

Si l'on pouvait démontrer directement ce Coroll. 2, en se servant du Coroll. à la Prop. 14.2 et de la Prop. 22.2, on en déduirait sans peine la Prop. 23.1 et le théorème de Levr. Il y a donc équivalence entre, d'une part, la Prop. 23.1 et le théorème de Levi et, d'autre part, le Coroll. 2 ci-dessus, le Coroll. à la Prop. 14.2 et la Prop. 22.2.

Proposimion 23.2. - Soit B un groupe de Lie semi-simple simplement connexe, $\mathrm{F}$ un groupe de Lie connexe et $\chi$ une représentation continue de $\mathrm{B}$ dans $\mathcal{B}(\mathrm{F})$, muni de la topologie quotient de la topologie de groupe de Lie de $\mathfrak{G}(\mathrm{F})$. Il existe alors une extension inessentielle $\mathrm{E}(\mathrm{B}, \mathrm{F})$ de caractère $\chi$.

Grâce au Coroll. 2 à la Prop. 13.2 il suffira de montrer qu'il existe une représentation continue $\xi$ de $B$ dans $\mathscr{Q}(F)$ qui se projette sur $\chi$. La proposition sera alors une conséquence du

LEMME 23.1. - Soit B un groupe de Lie semi-simple simplement connexe, 
$\mathrm{G}$ un groupe admettant un sous-groupe distingué $\mathrm{N}$ de Lie: toute représenta. tion continue $\mathrm{f}$ de $\mathrm{B}$ dans $\mathrm{G} / \mathrm{N}$ est la projection d'une représentation continue $\mathrm{f}^{\prime}$ de $\mathrm{B}$ dans $\mathrm{G}$.

En effet $f^{*} G$ est une extension de $N$ par $B$, qui est inessentielle d'après le théorème de LEVI: il y a done un isomorphisme de $B$ dans $f^{*} G$ qui, composé arec l'application canonique de $f^{*} G$ dans $G$, donne une représentation $f^{\prime}$ de $B$ dans $G$, qu' on voit se projeter sur $f$.

\section{\$ 24. - Les extensions locales.}

Il y a parfois intérêt, surtout pour les groupes de Lre, à considérer des groupes locaux: on pent faire pour eux une théorie des extensions locales. Pour les notions de groupe local, sous-groupe local etc. voir p. ex. [27], chap. $3, \$ 23$.

DeFinition 24.1 - B et F étant deux groupes locaux, une extension locale de F par B est définie par la donnée d'un groupe local $\mathrm{E}$ qui admet $\mathrm{F}$ comme sous-groupe local distingué fermé, et d'un isomorphisme local de $\mathrm{E} / \mathrm{F}$ sur $\mathrm{B}$.

Definitron 24.2 - Le groupe local $\mathrm{G}$ est dit prolongeable dans le groupe $\overline{\mathrm{G}} \mathrm{s}^{\prime}$ il existe un isomorphisme local de $\mathrm{G}$ sur un voisinage de l'élément neutre de $\overline{\mathrm{G}}$.

On peut démontrer la

Proposition 24.1 - Soit $\mathrm{E}(\mathrm{B}, \mathrm{F})$ une extension locale inessentielle et supposons que $\mathrm{B}$ et $\mathrm{F}$ soient prolongeables en $\overline{\mathrm{B}}$ et respectivement en $\overline{\mathrm{F}}$. Si ces deux groupes sont localement connexes ot simplement connexes, $\mathrm{E}$ est prolongeable en un groupe $\overline{\mathrm{E}}$ qui admet une structure d'extension inessentielle $\overline{\mathrm{E}}(\overline{\mathrm{B}}, \overline{\mathrm{F}})$ univoquement déterminée et localement équivalente $\grave{a} \mathrm{E}(\mathrm{B}, \mathrm{F})$.

Cette proposition contient comme cas particulier le Lemme de [27], chap. 9, \& 54 sur lequel Pontrjagin se base pour démontrer le troisième théorème de Lie dans le cas général.

\section{§ 25. - Les groupes non résolubles à $n \leq 4$ dimensions $\left({ }^{34}\right)$.}

Comme application de la théorie des extensions nous nous proposons d'établir la liste des groupes de LIE à $n \leq 4$ dimensions.

Pour les groupes non résolubles le problème est aisé. En effet il n'y a que deux groupes simples simplement connexes (cfr. [8]): la sphère $S_{3}$ et le groupe revètement simplement connexe $S \tilde{S L}$ de $S L(2, R)$; ils ont trois dimensions. Done tout groupe simplement connexe non résoluble et non simple est ì 4 dimensions et admet ane structure d'extension inessentielle de $R$ par $S_{3}$ ou par $S L$. On voit qu'elles sont toutes triviales, car $\mathfrak{Q}(R)=R$.

Lies extensions de $T$ par $S_{3}$ ou par $S \tilde{L}$ sont aussi triviales: elles sont en effet centrales (Prop. 11.4) et inessentielles (thé. de LEvi). Les extensions de $R$ par $P_{3}$ (quotient de $S_{3}$ par son centrel et par $S L(2, R)$ seront centrales et localement inessentielles; mais il n'y a pas de sous-groupes finis dans $R$, et done $E\left(P_{n}, R\right)$ est triviale.

A côté de l'extension triviale $S L \times R$, il y a encore ane extension $E_{4}(S L, R)$, admettant $\breve{S L}$ comme sous-groupe distingué : puisque $\breve{S L} \cap R=Z, E_{1}$ a aussi

$\left({ }^{4}\right)$ Ce paragraphe corrige et complète le paragraphe de même titre de [6]. 
une structure d'extension $E_{1}(T, \overleftarrow{S L})$. Ces deux extensions ne sont pas équivalentes à l'extension triviale (Prop. 15.1, Coroll. 2) et done elles ne lui sont non plus isomorphes $\left(\$ 7\right.$, note $\left.\left({ }^{15}\right)\right)$. D'autre part comme $R$ (resp. $\check{S} L$ ) est un sous-groupe caractéristique de $E_{1}$ il n'y a pas d'isomorphisme de $F_{1}$ sur $S L \times R$ (resp. sur $T \times \widehat{S L}$ ) qui ne soit pas un isomorphisme d'extension.

Le même reste vrai en remplaçant $S L$ par un de ses groupes revêtements connexes non simplement connexes; mais tous les groupes qu' on obtient ainsi sont isomorphes à $E_{1}$, ear ils ont tous nne structure $E(T, S L)$.

Rappelons que pour chaque $i, 1<i<\infty$, il y a un groupe revêtement connexe $\left.S L_{i}=\widehat{S L}_{i}\left(S L, Z_{i}\right){ }^{35}\right)$; si nous posons $\widehat{S L_{1}}=S L$, pour tout $1 \leq i$, $k<\infty$ on a une extension $E_{i, k}\left(\widehat{S L}_{i}, T\right)$ qui admet $\widehat{S L}_{i k}$ comme sous-groupe distingué: $E_{i, k} / \widehat{S L}_{i k}$ est isomorphe à $T . E_{i, k}$ est isomorphe à $\widehat{S L}_{j} \times T$ si et seulement si $i=j$ et $k=1$, et n' est isomorphe àcun autre $E_{j, l}$.

Enfin, à part le produit $P_{3} \times T$, il y a encore un groupe $E_{2}\left(P_{3}, T\right)$ non isomorphe à $P_{3} \times T$, qui admet $S_{3}$ comme sous-groupe distingué.

Résumant on obtient: les groupes de LTE connexes non résolubles et non simples à $n \leq 4$ dimensions sont: ou par $T$.

- les groupes produits d'un groupe simple à 3 dimensions par $R$

- les groupes $E_{1}(S L, R)=E_{1}(T, \breve{S L})$ et $E_{i, k}\left(\widehat{S L}_{i}, T\right)=E_{i, k}\left(\mathrm{~T}, \widehat{S L}_{i k}\right)$ pour $i \geq 1, k \geq 2$ : tous ces groupes ont les mềmes constantes de structure $c_{342}=1, c_{243}=-1, c_{234}=-1$ (les autres $=0$ ).

- le groupe $E_{3}\left(P_{3}, T\right)=E_{2}\left(T, S_{3}\right)$ dont les constantes de structure non nulles sont $c_{342}=1, c_{243}=-1, c_{234}=1$.

Comme il résulte de [13], \& 2, Prop. 2, $E_{\text {, }}$ et $E_{i, k}$ (resp. $E_{2}$ ) sont homéomorphes à $T^{2} \times R^{2}$ (resp. à $T \times S_{3}$ ). Done les gronpes de LIE connexes à 4 dimensions sont tous homéomorphes à l'un des espaces suivants: $R^{n} \times T^{s}$ avec $n+s=4, R \times S_{3}, R \times P_{3}, T \times S_{3}, T \times P_{3}$.

Plus généralement, on pent montrer facilement que tout groupe de LIE connexe à $n \leq 7$ dimensions est homéomorphe à un produit $R^{p} \times T^{q} \times P_{3}^{r} \times S_{3}^{s}$ avee $p+q+3 r+3 s=n$.

\section{\& 26. - Les groupes résolubles à $n \leq 3$ dimensions.}

Tout groupe $E$ résoluble simplement connexe à $n$ dimensions est une extension inessentielle d'un groupe $F$ résoluble simplement connexe à $n-1$ dimensions par $R$. Pour connaitre les groupes $E$ en se servant de la théorie des extensions, il suffit d'établir la liste des extensions $E(R, F)$ à un isomor. phisme près. Or le Coroll. à la Prop. 5.1, le Coroll. à la Prop. 13.2 et la

(35) $Z_{i}$ : groupe des entiers modulo $i$. 
Prop. 13.3 nous permettent d'affirmer $q \mathbf{u}^{\prime}$ il y a correspondance biunivoque entre les classes d'extensions $E(R, F)$ isomorphes et les classes de sous-groupes conjugués à une dimension de $\mathcal{E}(F)$, muni de la topologie quotient de la topologie de groupe de LIE de $\mathfrak{d}(F)$. Nous verrons que pour les petites dimen. sions la détermination de $\mathcal{E}(F)$, de ses sous-groupes à une dimension et des extensions correspondantes ne présente pas de difficultés particulières, encore que de lungs calculs soient nécessaires.

Lorsqu'on aura une liste des extensions $E(R, F)$ pour tous les groupes $F$ de dimension $n-1$, il faudra chercher les isomorphismes de groupe existant entre elles: on pourra ainsi trouver les structures de groupe à $n$ dimensions.

Dans les listes qui vont suivre nous nous limitons à donner les groupes simplement connexes, en indiquant leur centre lorsqu'il ne se réduit pas à l'élément neutre. De chaque groupe, qui est homéomorphe à $R^{n}$ et dans lequel nous avons choisi un système de coordonnées globales $x_{1}, x_{2}, \ldots, x_{n}$ foù les $x_{i}$ avec $i \geq 2$ sont les coordonnées de.$F$ ), nous donnons les coordonnées $\mathrm{du}$ composé $\left(x_{1}, x_{2}, \ldots, x_{n}\right)\left(x_{1}^{\prime}, x_{2}{ }^{\prime}, \ldots, x_{n}{ }^{\prime}\right)$, les constantes de structure non nulles et des structures d'extension que le groupe admet. La détermination des constantes de structure a été faite a posteriori par des calculs fort simples (cfr. \& 19) que nous ne reporterons pas ici.

$n=1$, 2. Les résultats sont bien connus: à part $R$ et $R^{2}$ il n'y a que le groupe des transformations affines de la droite, que nous noterons $A$ :

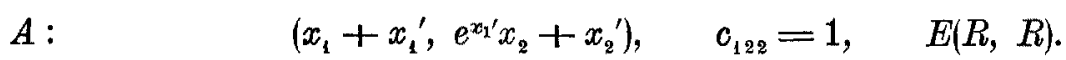

Ce groupe n'a pas de centre et tous ses automorphismes sont intérieurs: il n'y aura donc pas d'extension $E(R, A)$ non équivalente à l'extension triviale. C'est dire que tous les groúpes résolubles à 3 dimensions ont une structure $E\left(R, R^{2}\right)$. Comme $\mathfrak{A}\left(R^{2}\right)=\mathscr{E}\left(R^{2}\right)=G L(2, R)$, nous pouvons utiliser la Prop. 20.2 et nous borner à considérer les représentations de $R$ dans $G L(2, R)$ suivantes :

a) représentation constante

b) $\xi_{x}=\left(\begin{array}{ll}1 & 0 \\ 0 & e^{x}\end{array}\right), \quad \xi_{x}=\left(\begin{array}{ll}e^{x} & 0 \\ 0 & 1\end{array}\right)$

c) $\xi_{x}=\left(\begin{array}{ll}1 & 0 \\ x & 1\end{array}\right)$

d) $\xi_{x}=\left(\begin{array}{cc}e^{x} & 0 \\ 0 & e^{x}\end{array}\right)\left(\begin{array}{ll}1 & 0 \\ x & 1\end{array}\right)$

e) $\xi_{x}=\left(\begin{array}{rr}\cos x & -\sin x \\ \sin x & \cos x\end{array}\right)$

f) $\xi_{x}=\left(\begin{array}{ll}e^{x} & 0 \\ 0 & e^{r x}\end{array}\right), r \neq 0$

g) $\xi_{x}=\left(\begin{array}{ll}e^{r x} & 0 \\ 0 & e^{r x}\end{array}\right)\left(\begin{array}{lr}\cos x & -\sin x \\ \sin x & \cos x\end{array}\right), r \neq 0$. 
Il est en effet clair que les seuls sous-groupes sans facteur triangulaire (resp. diagonal) sont $a$ ), b), e), f) (resp. c)). On vérifie alors que les seals pro. duits possibles sont donnés par $d$ ) et $g$ ).

Les extensions correspondantes seront construites par la méthode du \& 13 . On obtient ainsi :

a) $R^{3}$

b) $R \times A$

c) $G_{1}:\left(x_{1}+x_{1}^{\prime}, x_{2}+x_{2}^{\prime}, x_{1}^{\prime} x_{2}+x_{3}+x_{3}^{\prime}\right), c_{123}=1$, centre $x_{1}=x_{2}=0$, $E\left(R, R^{2}\right), E\left(R^{2}, R\right)$

d) $G_{2}:\left(x_{1}+x_{1}^{\prime}, \quad e^{x_{1}^{\prime}} x_{2}+x_{2}^{\prime}, \quad e^{x_{1}^{\prime}} x_{1}^{\prime} x_{2}+e^{x_{2}^{\prime}} x_{3}+x_{3}^{\prime}\right), \quad c_{122}=1, \quad c_{123}=1$, $c_{133}=1, E\left(R, R^{2}\right), E(A, R)$

e) $G_{3}:\left(x_{1}+x_{1}^{\prime}, x_{2} \cos x_{1}^{\prime}-x_{3} \sin x_{1}^{\prime}+x_{2}^{\prime}, x_{2} \sin x_{1}^{\prime}+x_{1} \cos x_{1}^{\prime}+x_{3}^{\prime}\right)$, $c_{132}=-1, c_{123}=1$, centre $x_{1}=2 k \pi, x_{2}=x_{3}=0, E\left(R, R^{\prime \prime}\right)$

f) $G_{4, r}:\left(x_{1}+x_{1}^{\prime}, e^{x_{1}^{\prime}} x_{2}+x_{2}^{\prime}, e^{r x_{1}^{\prime}} x_{3}+x_{3}^{\prime}\right), c_{122}=1, c_{133}=r, r \neq 0$, $E\left(R, R^{2}\right), E(A, R)$

g) $G_{5, r}:\left(x_{1}+x_{1}^{\prime}, e^{r x_{1}^{\prime}}\left(x_{2} \cos x_{1}^{\prime}-x_{3} \sin x_{1}^{\prime}\right)+x_{2}^{\prime}, e^{r x_{1}^{\prime}}\left(x_{2} \sin x_{1}^{\prime}+x_{3} \cos x_{1}^{\prime}\right)+x_{3}^{\prime}\right)$, $c_{122}=r, c_{132}=-1, c_{323}=1, c_{133}=r, r \neq 0$. Pour $r>0$ la transfor mation $\bar{x}_{1}=-x_{1}, \bar{x}_{2}=x_{2}, \bar{x}_{3}=x_{3}$, est un isomorphisme de $G_{5}$, sur $G_{5},-r$. Nous pourons done supposer $r>0 . E\left(R, R^{2}\right)$.

Cette liste est déjà réduite $\left.{ }^{36}\right)$ : deux des groupes qui y figurent ne sont jamais isomorphes. En effet les seuls groupes dont le sous-groupe des commutateurs soit isomorphe à $R$ sont $A \times R$ et $G_{1}$ : mais leurs' centres ont dimensions différentes. Pour les antres groupes, tout isomorphisme serait un isomorphisme d'extension sur $R$, car $R^{2}$ est earactéristique: il faudrait que les sous-groupes correspondants de $G L(2, R)$ fussent conjugués.

\section{\$ 27. - Les groupes des automorphismes des groupes résolubles simplement connexes à 3 dimensions.}

Les groupes résolubles simplement connexes à 4 dimensions que nous voulons trouver sont des extensions inessentielles par $R d^{\prime}$ un des groupes donnés au paragraphe précédent: il nous faut done en déterminer les auto. morphismes.

Lorsqu' on connaît un système de coordonnées d'un groupe simplement connexe, on peut déterminer ses automorphismes en ecrivant la transforma. tion la plus générale du système de coordonnées, qui soit compatible avec la loi de composition: on obtient des équations fonctionnelles qui sont facilement rosolubles dans les cas qui nous intéressent.

(36) Une liste des groupes résolubles à 3 dimensions a été donnée par LeE dans [Q4]. Les types VI et $B$ de LeE sont des groupes $G_{4, r}$ : pour $|r|<1, G_{4}, r$ ne figure pas chez LEE. Une autre liste figure dans [22]: elle parait aussi incomplète. 
I. $-R^{3}: \mathfrak{A}\left(R^{3}\right)=\mathfrak{G}\left(R^{3}\right)=G L(3, R)$. La Prop. 20.2 va nous permettre de déterminer un sous-groupe à une dimension de $G L(3 ; R)$ dans chaque classe de sous-groupes conjugués.

Si $\sigma(x)$ parcourt un sous-groupe sans facteur triangulaire, il existe une matrice $\left(\alpha_{i j}+i \beta_{i j}\right) \varepsilon G L(3, C)$ telle que

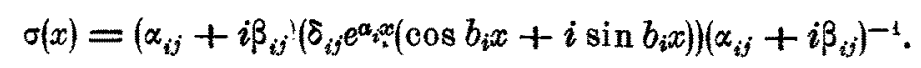

Posons $\quad x=\left(\alpha_{i j}\right), \quad \beta=\left(\beta_{i j}\right), \quad c(x)=\left(\delta_{i j} e^{a_{i} x} \cos b_{i} x\right), \quad s(x)=\left(\delta_{i j} e^{a_{i} x} \sin b_{i} x\right)$ et désignons par $\alpha^{*}$ et $\beta^{*}$ les deux matrices réelles telles que $\alpha^{*}+i \beta^{*}=(\alpha+i \beta)^{-1}$. En exprimant que les parties imaginaires de (27.1) sont égales on a

ou

$$
0=\beta c(x) \alpha^{*}+\alpha s(x) \alpha^{*}+\alpha c(x) \beta^{*}-\beta s(x) \beta^{*}
$$

$$
\begin{aligned}
& \beta c(x) \alpha^{*}+\alpha c(x) \beta^{*}=0 \\
& \alpha s(x) \alpha^{*}-\beta s(x) \beta^{*}=0 .
\end{aligned}
$$

D'autre part on a $(\alpha+i \beta)\left(\alpha^{*}+i \beta^{*}\right)=$ matrice unité, et donc

$$
\alpha \alpha^{*}-\beta \beta^{*}=\text { matrice unite. }
$$

(27.3) peut s' ecrire

$$
\sum_{k=1}^{3}\left(\alpha_{i k} \alpha_{k j}^{*}-\beta_{i k} \beta_{k i j}^{*}\right) e^{\alpha_{k} x} \sin b_{k} x=0 .
$$

On voit que si $b_{1}=b_{2}=b_{3} \neq 0,(27.5)$ entraine $\alpha \alpha^{*}-\beta \beta^{*}=0$, contraire. ment à (27.4). Donc, supposant $b, \neq 0$, après une permutation éventuelle on a certainement $b_{1} \neq 1$ : posons $x=\pi / 2 b_{1}+2 n \pi$ :

$$
\sum_{k=2}^{3}\left(\alpha_{i k} \alpha_{k j}^{*}-\beta_{i \hbar} \beta_{k j}^{*}\right) e^{a_{k}\left(\pi / 2 b_{1}+2 n \pi\right)} \sin b_{k}\left(\pi / 2 b_{1}+2 n \pi\right)=0 .
$$

Ce qui entratne $\alpha_{i k} \alpha_{k j}^{*}-\beta_{i k} \beta_{k j}^{*}=0, k=2,3:$ mais alors (27.5) donne aussi $\alpha_{i 1} \alpha_{1 j}^{*}-\beta_{t i} \beta_{1 j}^{*}=0$ et on a de nouveau contradiction.

Il y a donc un $i$ tel que $b_{i}=0$ : supposons que ce. soit $b_{3} . \sigma(x)$ est donc conjugué à une matrice de la forme

$$
\left(\begin{array}{lll}
e^{\left(a_{1}+i b_{1}\right) x x} & & \\
& e^{\left(a_{2}+i b_{2}\right) x x} & \\
& & e^{a_{3} x}
\end{array}\right)
$$

et nous sommes ainsi ramenés au cas de 2 variables. Remarquons que supposer $b_{3}=0$ n'est pas une restriction, car c'est équivalent à faire un changement de coordonnées dans $R^{3}$. Cette remarque nous permettra aussi d'abré. ger la liste qui va suivre. 
Utilisant le $\$ 26$, voici donc les représentations de $R$ dans $G L(3, R)$ sans facteur triangulaire:

a) application constante.

b) $\xi_{x}=\left(\begin{array}{cc}e^{x} & \\ & 1 \\ & 1\end{array}\right)$

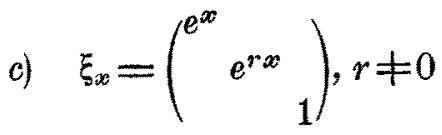

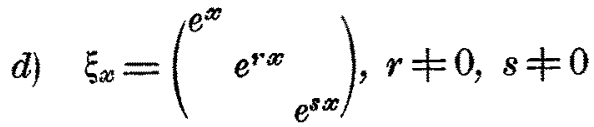

e) $\quad \xi_{x}=\left(\begin{array}{rr}\cos x & -\sin x \\ \sin x & \cos x \\ & \end{array}\right)$

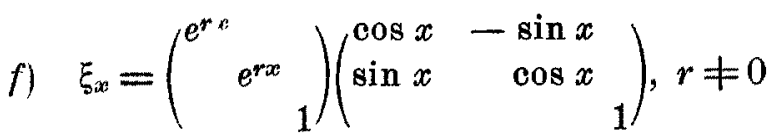

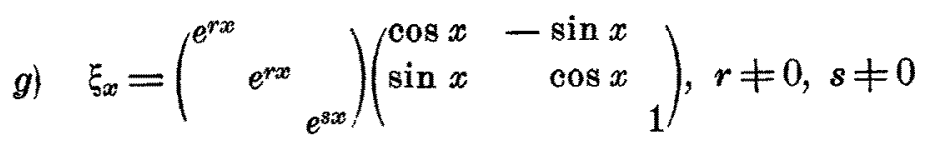

h) $\xi_{x}=\left(\begin{array}{lll}1 & & \\ & 1 & \\ & & e^{r r}\end{array}\right)\left(\begin{array}{rrr}\cos x & -\sin x \\ \sin x & \cos x \\ & & 1\end{array}\right), r \neq 0$.

Les représentations sans facteur diagonal sont données explicitement par la Prop. 20.2 :

$$
\text { i) } \begin{aligned}
\xi_{x} & =\left(\begin{array}{lll}
1 & & \\
x & 1 & \\
& & 1
\end{array}\right) \\
\text { j) } \xi & =\left(\begin{array}{ccc}
1 & & \\
x & 1 & \\
\frac{1}{2} x^{2} & x & 1
\end{array}\right) .
\end{aligned}
$$

Pour qu' une représentátion diagonale et une triangulaire puissent donner par multiplication une autre représentation, il faut et il suffit qu' elles 
commutent. On troure ainsi:

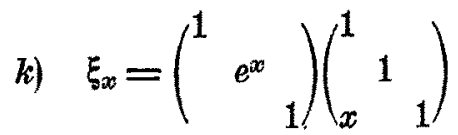

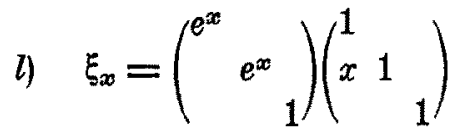
m) $\xi_{x}=\left(\begin{array}{lll}e^{x} & & \\ & e^{x} & \\ & & e^{r x}\end{array}\right)\left(\begin{array}{lll}1 & & \\ x & 1 & \\ & & 1\end{array}\right), r \neq 0$
n) $\xi_{x}=\left(\begin{array}{lll}e^{x} & & \\ & e^{x} & \\ & & e^{x}\end{array}\right)\left(\begin{array}{llll}1 & & & \\ x & & 1 & \\ \frac{1}{2} x^{2} & x & 1\end{array}\right)$.

II. $-R \times A:\left(x_{1}+x_{1}^{\prime}, x_{2}+x_{2}^{\prime}, e^{x_{2}^{\prime}} x_{3}+x_{3}^{\prime}\right)$. La restriction à $A$ d'un automorphisme de $R \times A$ est intérieur (§ 26) donc de la forme

$$
\left(0, x_{2}, x_{3}\right) \rightarrow\left(f\left(0, x_{2}, x_{3}\right), x_{2}, \gamma\left(e^{x_{2}}-1\right)+\delta x_{3}\right) .
$$

D'autre part les sous-groupes $x_{2}=x_{3}=0$ (centre) et $x_{1}=x_{2}=0$ (sousgronpe des commutateurs) sont caractéristiques: donc un automorphisme sera de la forme

$$
\left(x_{1}, x_{2}, x_{3}\right) \rightarrow\left(f\left(x_{1}, x_{2}\right), x_{2}, \gamma\left(e^{x_{2}}-1\right)+\delta x_{3}\right) .
$$

En écrivant que $f$ est compatible avec la loi de composition on trouve

$$
\left(x_{1}, x_{2}, x_{3}\right)-\left(\alpha x_{1}+\beta x_{2}, x_{2}, \gamma\left(e^{x_{2}}-1\right)+\delta x_{3}\right) .
$$

En applicant successivement deux automorphismes, que nous noterons respectivement $(\alpha, \beta, \gamma, \delta)$ et $\left(\alpha^{\prime}, \beta^{\prime}, \gamma^{\prime}, \delta^{\prime}\right)$, on trouve la loi de composition dans $\mathfrak{G}(R \times A)$ :

$$
\left(\alpha^{\prime}, \beta^{\prime}, \gamma^{\prime}, \delta^{\prime}\right)(\alpha, \beta, \gamma, \delta)=\left(\alpha^{\prime} \alpha, \alpha^{\prime} \beta+\beta^{\prime}, \gamma^{\prime}+\delta^{\prime} \gamma, \delta \delta^{\prime}\right),
$$

ce qui exprime que $\mathfrak{E}(R \times A)$ est isomorphe à $A \times A$. Les automorphismes intérieurs sont donnés par $\alpha=1, \beta=0$; ainsi $\mathfrak{E}(R \times A) \approx A \subset \mathfrak{Q}(R \times A)$. C'est dire que $\mathscr{G}(R \times A)$ est le groupe des matrices

$$
\left(\begin{array}{ll}
\alpha & \beta \\
0 & 1
\end{array}\right) .
$$


Voici donc les représentations de $R$ :

o) application constante

p) $\xi_{x}=\left(\begin{array}{ll}1 & x \\ 0 & 1\end{array}\right)$

q) $\xi_{x}=\left(\begin{array}{ll}e^{x} & 0 \\ 0 & 1\end{array}\right)$.

III. $-G_{1}:\left(x_{1}+x_{1}^{\prime}, x_{2}+x_{2}^{\prime}, x_{1}^{\prime} x_{2}+x_{3}+x_{\dot{3}}{ }^{\prime}\right)$. Le sous-groupe $x_{1}=x_{2}=0$ étant caractéristique, tout automorphisme sera de la forme

$$
\left(x_{1}, x_{2}, x_{3}\right) \rightarrow\left(\alpha x_{1}+\beta x_{2}, \gamma x_{1}+\delta r_{2}, f\left(x_{1}, x_{2}, x_{3}\right)\right)
$$

où $f$ doit satisfaire l'équation

$$
\begin{gathered}
f\left(x_{1}+x_{1}^{\prime}, x_{2}+x_{2}^{\prime}, x_{1}^{\prime} x_{2}+x_{3}+x_{3}^{\prime}\right)= \\
=\left(\alpha x_{1}^{\prime}+\beta x_{2}^{\prime}\right)\left(\gamma x_{1}+\delta x_{2}\right)+f\left(x_{1}, x_{2}, x_{3}\right)+f\left(x_{1}^{\prime}, x_{2}^{\prime}, x_{3}\right) .
\end{gathered}
$$

En particulier done

$$
f\left(x_{1}+x_{1}^{\prime}, 0,0\right)=\alpha \gamma x_{1} x_{1}^{\prime}+f\left(x_{1}, 0,0\right)+f\left(x_{1}^{\prime}, 0,0\right)
$$

ce qu'on peut érire

$$
\frac{f\left(x_{1}+x_{1}^{\prime}, 0,0\right)-f\left(x_{1}, 0,0\right)}{x_{1}^{\prime}}=\alpha \gamma x_{1}+\frac{f\left(x_{1}^{\prime}, 0,0\right)}{x_{1}^{\prime}} .
$$

$f$ étant continùment dérivable, on voit que

$$
\frac{\partial f\left(x_{3}, 0,0\right)}{\partial x_{1}}=\alpha \gamma x_{1}+\varepsilon
$$

et donc que $f\left(x_{1}, 0,0\right)=\frac{1}{2} \alpha \gamma x_{1}^{2}+\varepsilon x_{1}$. D' une façon analogue on trouve $f\left(0, x_{2}, 0\right)=\frac{1}{2} \beta \delta x_{2}^{2}+\vartheta x_{2}$ et, evidemment, $f\left(0,0, x_{3}\right)=\eta x_{3}$.

Or

$$
\begin{aligned}
\beta \gamma x_{1} x_{2}+f\left(x_{1}, 0,0\right)+f\left(0, x_{2}, 0\right) & =f\left(x_{1}, x_{2}, 0\right) \\
\alpha \delta x_{1} x_{2}+f\left(0, x_{2}, 0\right)+f\left(x_{1}, 0,0\right) & =f\left(x_{1}, x_{2}, x_{1} x_{2}\right) \\
f\left(x_{1}, x_{2}, 0\right)+f\left(0,0 x_{3}\right) & =f\left(x_{1}, x_{2}, x_{3}\right) .
\end{aligned}
$$

Il en résulte immédiatement que l'automorphisme générique est $\left(x_{1}, x_{2}, x_{3}\right) \rightarrow\left(\alpha x_{1}+\beta x_{2}, \gamma x_{1}+\delta x_{2}, \frac{1}{2} \alpha \gamma x_{1}^{2}+\varepsilon x_{1}+\frac{1}{2} \beta \delta x_{2}^{2}+\delta x_{2}+\beta \gamma x_{1} x_{2}+(\alpha \delta-\beta \gamma) x_{3}\right)$

La loi de composition est $\left(\alpha \alpha^{\prime}+\beta^{\prime} \gamma, \alpha^{\prime} \beta+\delta \beta^{\prime}, \delta^{\prime} \gamma+\alpha \gamma^{\prime}, \delta \delta^{\prime}+\gamma^{\prime} \beta, \beta \vartheta^{\prime}+\delta \varepsilon^{\prime}+\left(\alpha^{\prime} \delta^{\prime}-\beta^{\prime} \gamma^{\prime}\right) \varepsilon, \alpha \vartheta^{\prime}+\varepsilon^{\prime} \gamma+\left(\alpha^{\prime} \delta^{\prime}-\beta^{\prime} \gamma^{\prime}\right) \vartheta\right)$ 
et done $\mathfrak{A}\left(G_{1}\right)$ est le gronpe des matrices

$$
\left(\begin{array}{ccc}
\alpha^{\xi}-\beta \gamma & \vartheta & \varepsilon \\
0 & \alpha & \beta \\
0 & \gamma & \delta
\end{array}\right) .
$$

Pour les automorphismes intérieurs on aura certainement $\alpha=\delta=1$, $\beta=\gamma=0$; d'autre part nous savons déjà que $\mathfrak{J}\left(G_{1}\right)=G_{1} /$ centre. Ils sont donc caractérisés par ces égalités et $\mathfrak{g}\left(G_{1}\right)$ sera fermé. Ainsi $\mathscr{G}\left(G_{1}\right)=G L(2, R)$. Toute représentation $\chi$ de $R$ dans $\mathcal{G}\left(G_{1}\right)$ étant la projection d'une représen. tation $\xi$ dans $\mathcal{Q}\left(G_{1}\right)$, en voici la liste:

r) application constante

$$
\begin{aligned}
& \text { s) } \xi_{x}=\left(\begin{array}{lll}
e^{x} & & \\
& e^{x} & \\
& & 1
\end{array}\right) \\
& \text { t) } \xi_{x}=\left(\begin{array}{lll}
e^{(r+1) x} & & \\
& e^{x} & \\
& & e^{r x}
\end{array}\right), r \neq 0 \\
& \text { u) } \xi_{x}=\left(\begin{array}{rrr}
1 & & \\
\cos x & -\sin x \\
\sin x & \cos x
\end{array}\right) \\
& \text { v) } \xi_{x}=\left(\begin{array}{lll}
e^{2 r x} & & \\
& e^{r x x} & \\
& & e^{r x}
\end{array}\right)\left(\begin{array}{rrr}
1 & & \\
& \cos x & -\sin x \\
\sin x & \cos x
\end{array}\right), r \neq 0 \\
& \text { w) } \xi_{x}=\left(\begin{array}{lll}
1 & & \\
& 1 & \\
& x & 1
\end{array}\right) \\
& \text { y) } \xi_{x}=\left(\begin{array}{lll}
e^{2 x} & & \\
& e^{x} & \\
& & e^{x}
\end{array}\right)\left(\begin{array}{lll}
1 & & \\
& 1 & \\
& x & 1
\end{array}\right) \text {. }
\end{aligned}
$$

Maintenant nous ne pouvons pas changer arbitrairement le système de coordonnées dans $G$ : il fandra donc considérer aussi

s) $\xi_{x}=\left(\begin{array}{lll}e^{x} & & \\ & 1 & \\ & & e^{x}\end{array}\right)$.

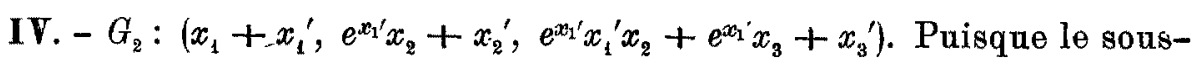
groupe $x_{i}=0$ est caractéristique, un automorphisme sera donné par

$$
\left(x_{1}, x_{2}, x_{3}\right) \rightarrow\left(\alpha x_{1}, f\left(x_{1}, x_{2}, x_{3}\right), g\left(x_{1}, x_{2}, x_{3}\right)\right)
$$


où

$$
\begin{aligned}
& \left.e^{\alpha x_{1}} f\left(x_{1}, x_{2}, x_{3}\right)+f x_{1}^{\prime}, x_{2}^{\prime}, x_{3}{ }^{\prime}\right)=
\end{aligned}
$$

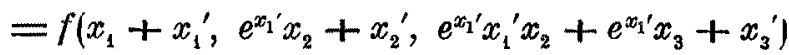

$$
\begin{aligned}
& \alpha e^{x x_{1} x_{1}^{\prime}} f\left(x_{1}, x_{2}, x_{3}\right)+e^{\alpha x_{1}^{\prime}} g\left(x_{1}, x_{2}, x_{3}\right)+g\left(x_{1}^{\prime}, x_{2}^{\prime}, x_{3}\right)=
\end{aligned}
$$

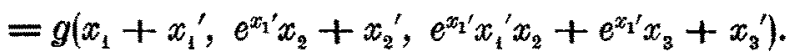

En posant $x_{2}=x_{3}=x_{2}{ }^{\prime}=x_{3}{ }^{\prime}=0$ dans (27.6) on trouve

$$
\begin{aligned}
e^{\alpha x_{1}{ }^{\prime}} f\left(x_{1}, 0,0\right)+f\left(x_{1}{ }^{\prime}, 0,0\right) & =f\left(x_{1}+x_{1}{ }^{\prime}, 0,0\right)= \\
& =e^{x x_{1}} f\left(x_{1}{ }^{\prime}, 0,0\right)+f\left(x_{1}, 0,0\right)
\end{aligned}
$$

d'où $f\left(x_{1}, 0,0\right)=\beta\left(e^{\alpha x_{2}}-1\right)$. Si $x_{1}=x_{1}{ }^{\prime}=0$, on reconnatt que $f\left(0, x_{2}, x_{i}\right)=$ $=\gamma x_{2}+\delta x_{3} ;$ et puisque

on obtient

$$
f\left(x_{1}, 0,0\right)+f\left(0, x_{2}, x_{3}\right)=f\left(x_{1}, x_{2}, x_{3}\right)
$$

$$
f\left(x_{1}, x_{2}, x_{3}\right)=\beta\left(e^{\alpha x_{1}}-1\right)+\gamma x_{2}+\delta x_{3} .
$$

Mais il faut aussi aroir

$$
e^{x x_{1}} f\left(0, x_{2}, x_{3}\right)+f\left(x_{1}, 0,0\right)=f\left(x_{1}, e^{x_{1}} x_{2}, e^{x_{2}} x_{1} x_{2}+e^{x_{1}} x_{3}\right)
$$

ce qui entraîne nécessairement $\alpha=1, \delta=0$. Donc enfin $f\left(x_{1}, x_{2}, x_{3}\right)=$ $=\beta\left(e^{x_{1}}-1\right)+\gamma x_{2}$. On est maintenant en mesure de calculer $g$ grâce à (27.7): on le fera par un procédé en tout analogue et l'on trouvera pour finir, en changeant les constantes,

$$
\left(x_{1}, x_{2}, x_{3}\right) \rightarrow\left(x_{1}, \alpha\left(e^{x_{1}}-1\right)+\beta x_{2}, \alpha e^{x_{1}} x_{1}+\gamma\left(e^{x_{1}}-1\right)+\delta x_{2}+\beta x_{3}\right) .
$$

La loi de composition entre les automorphismes est donnée par

$$
\left(\beta^{\prime} \alpha+\alpha^{\prime}, \beta \beta^{\prime}, \alpha \delta^{\prime}+\beta^{\prime} \gamma+\gamma^{\prime}, \beta^{\prime} \delta+\beta \delta^{\prime}\right) ;
$$

Les éléments de $\mathfrak{A}\left(G_{2}\right)$ sont donc les matrices

$$
\left(\begin{array}{lll}
\beta & \delta & \gamma \\
0 & \beta & \alpha \\
0 & 0 & 1
\end{array}\right)
$$

Si l'on calcule alors les automorphismes intérieurs, on voit que $\mathfrak{d}\left(G_{2}\right)$ est caractérisé par la relation $\delta=\beta \lg \beta$, et $q u$ 'il est fermé. Donc $\mathscr{G}\left(G_{2}\right)=R$. On aura seulement les représentations:

aa) application constante

ab) $\xi_{x}=\left(\begin{array}{lll}1 & x & \\ & 1 & \\ & & 1\end{array}\right)$. 
V. $-G_{3}:\left(x_{1}+x_{1}^{\prime}, x_{2} \cos x_{1}^{\prime}-x_{3} \sin x_{1}^{\prime}+x_{2}^{\prime}, x_{2} \sin x_{1}^{\prime}+x_{3} \cos x_{1}^{\prime}+x_{3}{ }^{\prime}\right)$. Comme plus haut, les automorphismes seront de la forme

$\operatorname{avec}$

$$
\left(x_{1}, x_{2}, x_{3}\right) \rightarrow\left(\alpha x_{1}, f\left(x_{1}, x_{2}, x_{3}\right), g\left(x_{1}, x_{2}, x_{3}\right)\right)
$$

$$
\begin{aligned}
& f\left(x_{1}, x_{2}, x_{3}\right) \cos \alpha x_{1}^{\prime}-g\left(x_{1}, x_{2}, x_{3}\right) \sin \alpha x_{1}^{\prime}+f\left(x_{1}^{\prime}, x_{2}^{\prime}, x_{3}^{\prime}\right)= \\
= & f\left(x_{1}+x_{1}^{\prime}, x_{2} \cos x_{1}^{\prime}-x_{3} \sin x_{1}^{\prime}+x_{2}^{\prime}, x_{2} \sin x_{1}^{\prime}+x_{3} \cos x_{1}^{\prime}+x_{3}^{\prime}\right) \\
& f\left(x_{1}, x_{2}, x_{3}\right) \sin \alpha x_{1}^{\prime}+g\left(x_{1}, x_{2}, x_{3}\right) \cos \alpha x_{1}^{\prime}+g\left(x_{1}^{\prime}, x_{2}^{\prime}, x_{3}^{\prime}\right)= \\
= & g\left(x_{1}+x_{1}^{\prime}, x_{2} \cos x_{1}^{\prime}-x_{3} \sin x_{1}^{\prime}+x_{2}^{\prime}, x_{2} \sin x_{1}^{\prime}+x_{3} \cos x_{1}^{\prime}+x_{3}^{\prime}\right):
\end{aligned}
$$

d'où immédiatement

$$
\begin{aligned}
& f\left(0, x_{2}, x_{3}\right)=\beta x_{2}+\gamma x_{3} \\
& g\left(0, x_{2}, x_{3}\right)=\delta x_{2}+\varepsilon x_{3} .
\end{aligned}
$$

D'autre part (27.8) devient un système en $f\left(x_{1}, 0,0\right)$ et $g\left(x_{1}, 0,0\right)$ si l'on pose $x_{2}=x_{3}=x_{2}^{\prime}=x_{3}^{\prime}=0$ et si l'on fixe $x_{1}^{\prime}$. Additionnant et soustrayant terme à terme, et résolvant, on obtient

Puisque

$$
\begin{aligned}
& f\left(x_{1}, 0,0\right)=\vartheta \cos \alpha x_{1}+\eta \sin \alpha x_{1}-\vartheta \\
& g\left(x_{1}, 0,0\right)=-\eta \cos \alpha x_{1}+\vartheta \sin \alpha x_{1}+\eta
\end{aligned}
$$

on a

$$
\begin{aligned}
& f\left(x_{1}, 0,0\right)+f\left(0, x_{2}, x_{3}\right)=f\left(x_{1}, x_{2}, x_{3}\right) \\
& g\left(x_{1}, 0,0\right)+g\left(0, x_{2}, x_{3}\right)=g\left(x_{1}, x_{2}, x_{3}\right)
\end{aligned}
$$

$$
\begin{gathered}
f\left(0, x_{2}, x_{3}\right) \cos \alpha x_{1}-g\left(0, x_{2}, x_{3}\right) \sin \alpha x_{1}+f\left(x_{1}, 0,0\right)= \\
=f\left(x_{1}, x_{2} \cos x_{1}-x_{3} \sin x_{1}, x_{2} \sin x_{1}+x_{3} \cos x_{1}\right)= \\
=f\left(x_{1}, 0,0\right)+f\left(0, x_{2} \cos x_{1}-x_{3} \sin x_{1}, x_{2} \sin x_{1}+x_{3} \cos x_{1}\right)
\end{gathered}
$$

donc

$$
\begin{gathered}
\left(\beta x_{2}+\gamma x_{3}\right) \cos \alpha x_{1}-\left(\delta x_{2}+\varepsilon x_{3}\right) \sin \alpha x_{1}= \\
=\beta x_{2} \cos x_{1}-\beta x_{3} \sin x_{1}+\gamma x_{2} \sin x_{1}+\gamma x_{3} \cos x_{1},
\end{gathered}
$$

et, d'une façon analogue,

$$
\begin{gathered}
\left(\beta x_{2}+\gamma x_{3}\right) \sin \alpha x_{1}+\left(\delta x_{2}+\varepsilon x_{3}\right) \cos \alpha x_{1}= \\
=\delta x_{2} \cos x_{1}-\delta x_{3} \sin x_{1}+\varepsilon x_{2} \sin x_{1}+\varepsilon x_{3} \cos x_{1} .
\end{gathered}
$$

Pour $x_{1}=2 n \pi / \alpha$, il faut aroir $\cos (2 n \pi / \alpha)=1$, pour tout $n$. Done $\alpha=1$. D' où $\gamma=-\delta, \beta=\varepsilon$.

On arrive au résultat, avec d'autres constantes,

$$
\begin{array}{r}
\left(x_{1}, x_{2}, x_{3}\right) \rightarrow\left(x_{1}, \alpha \cos x_{1}+\beta \sin x_{1}-\alpha+\gamma x_{2}+\delta x_{3},\right. \\
\left.-\beta \cos x_{1}+\alpha \sin x_{1}+\beta-\delta x_{2}+\gamma x_{3}\right)
\end{array}
$$


La loi de composition est donnée par

$$
\left(\gamma^{\prime} \alpha+\alpha^{\prime}-\delta \beta, \beta^{\prime}+\gamma^{\prime} \beta+\delta^{\prime} \alpha, \gamma \gamma^{\prime}-\delta \delta^{\prime}, \gamma^{\prime} \delta+\gamma \delta^{\prime}\right) ;
$$

c'est-à-dire $\mathfrak{Q}\left(G_{3}\right)$ est le groupe des matrices

$$
\left(\begin{array}{rrr}
\gamma & -\delta & \alpha \\
\delta & \gamma & \beta \\
0 & 0 & 1
\end{array}\right) .
$$

$\mathfrak{d}\left(G_{3}\right)$ est caractérisé par $\gamma=\cos \varphi, \delta=\sin \varphi$. Nous pouvons prendre pour $\mathcal{E}\left(G_{3}\right)=R$ le sous-groupe caractérisé par $\alpha=\beta=\delta=0$.

ac) application constante

ad) $\xi_{x}=\left(\begin{array}{lll}e^{x} & & \\ & e^{x} & \\ & & 1\end{array}\right)$.

VI. $-G_{4, n}:\left(x_{1}+x_{1}^{\prime}, e^{x_{1}{ }^{\prime}} x_{2}+x_{2}^{\prime}, e^{r x_{1}{ }^{\prime}} x_{3}+x_{3}^{\prime}\right)$. On a toujours

avec

$$
\left(x_{1}, x_{2}, x_{3}\right) \rightarrow\left(\alpha x_{1}, f\left(x_{1}, x_{2}, x_{3}\right), g\left(x_{1}, x_{2}, x_{3}\right)\right)
$$

$e^{\alpha, x_{1}{ }^{\prime}} f\left(x_{1}, x_{2}, x_{2}\right)+f\left(x_{1}{ }^{\prime}, x_{2}{ }^{\prime}, x_{3}\right)=f\left(x_{1}+x_{1}{ }^{\prime}, e^{x_{1}{ }^{\prime}} x_{2}+x_{2}{ }^{\prime}, e^{r x_{1}{ }^{\prime}} x_{3}+x_{3}{ }^{\prime}\right)$

$\left.e^{x r x_{1}} g x_{1}, x_{2}, x_{3}\right)+g\left(x_{1}{ }^{\prime}, x_{2}{ }^{\prime}, x_{3}{ }^{\prime}\right)=g\left(x_{1}+x_{1}{ }^{\prime}, e^{x_{2}{ }^{\prime}} x_{2}+x_{2}{ }^{\prime}, e^{r x_{1}{ }^{\prime}} x_{3}+x_{3}{ }^{\prime}\right)$.

Comme pour $G_{2}$ on trouve sans difficulté

ainsi que la relation

$$
\begin{aligned}
& f\left(x_{1}, 0,0\right)=\beta\left(e^{x x_{1}}-1\right) \\
& f\left(0, x_{2}, x_{3}\right)=\gamma x_{2}+\delta x_{3} \\
& g\left(x_{1}, 0,0\right)=\varepsilon\left(e^{x r x_{1}}-1\right) \\
& g\left(0, x_{2}, x_{3}\right)=\Im x_{2}+\eta x_{3}
\end{aligned}
$$

$$
\begin{gathered}
e^{x x_{1}} f\left(0, x_{2}, x_{3}\right)+f\left(x_{1}, 0,0\right)=f\left(x_{1}, e^{x_{1}} x_{2}, e^{r x_{1}} x_{3}\right)= \\
=f\left(x_{1}, 0,0\right)+f\left(0, e^{x_{1}} x_{2}, e^{r x_{1}} x_{3}\right)
\end{gathered}
$$

et la relation analogue pour $g$.

Pour $r=1$ il en suit immédiatement $\alpha=1$ : donc, avec d'autres censtantes,

$\left(x_{1}, x_{2}, x_{3}\right) \rightarrow\left(x_{1}, \alpha\left(e^{x_{1}}-1\right)+\beta x_{2}+\gamma x_{3}, \delta\left(e^{x_{1}}-1\right)+\varepsilon x_{2}+\delta x_{3}\right) \quad r=1$.

Pour $r \neq 1$, on obtient $\alpha=1, \delta=\vartheta=0:$ done

$$
\left(x_{1}, x_{2}, x_{3}\right) \rightarrow\left(x_{1}, \alpha\left(e^{x_{1}}-1\right)+\beta x_{2}, \gamma\left(e^{r x_{1}}-1\right)+\delta x_{3}\right) \quad r \neq 1 .
$$

$r=1$. La loi de composition entre les automorphismes est. donnée par $\left(\beta^{\prime} \alpha+\alpha^{\prime}+\gamma^{\prime} \delta, \beta \beta^{\prime}+\gamma^{\prime} \varepsilon, \beta^{\prime} \gamma+\gamma^{\prime} \vartheta, \alpha \varepsilon^{\prime}+\delta^{\prime}+\vartheta^{\prime} \delta, \beta \varepsilon^{\prime}+\varepsilon \vartheta^{\prime}, \varepsilon^{\prime} \gamma+\vartheta \vartheta^{\prime}\right)$ 
et les automorphismes intérieurs par $\gamma=\varepsilon=0, \beta=\vartheta$. Ainsi $\mathfrak{A}\left(G_{4,1}\right)$ est le groupe des matrices

$$
\left(\begin{array}{lll}
\beta & \gamma & \alpha \\
\varepsilon & \vartheta & \delta \\
0 & 0 & 1
\end{array}\right)
$$

et l'on a à considérer les représentations :

ae) application constante

af) $\xi_{x}=\left(\begin{array}{lll}e^{x} & & \\ & 1 & \\ & & 1\end{array}\right)$

ag) $\xi_{x}=\left(\begin{array}{lll}1 & & \\ x & 1 & \\ & & 1\end{array}\right)$

ah) $\xi_{x}=\left(\begin{array}{lll}e^{x} & & \\ & e^{x} & \\ & & 1\end{array}\right)\left(\begin{array}{lll}1 & & \\ x & 1 & \\ & & 1\end{array}\right)$

ai) $\xi_{x}=\left(\begin{array}{rrr}\cos x & -\sin x & \\ \sin x & \cos x & \\ & & 1\end{array}\right)$

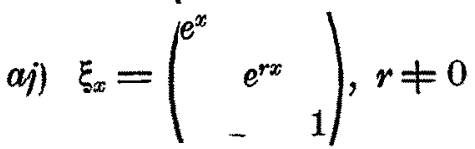

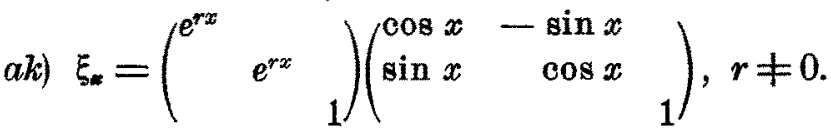

Vu la symétrie entre les sous-groupes $x_{1}=x_{3}=0$ et $x_{1}=x_{i}=0$, il n'y a pas d'autres représentations à ajouter.

$r \neq 1$. La loi de composition est

$$
\left(\beta^{\prime} \alpha+\alpha^{\prime}, \beta \beta^{\prime}, \delta^{\prime} \gamma+\gamma^{\prime}: \delta \delta^{\prime}\right),
$$

c' est-à-dire que $\mathfrak{Q}\left(G_{1}, r\right)$ est le groupe des matrices

$$
\left(\begin{array}{lll}
\beta & 0 & \alpha \\
0 & \delta & \gamma \\
0 & 0 & 1
\end{array}\right)
$$

$\mathfrak{T}\left(\mathrm{G}_{i},.\right)$ est caractérisé par $\delta=\beta^{r}$. Il y a donc les représentations :

al) application constante

am) $\xi_{x}=\left(\begin{array}{lll}1 & & \\ & e^{x} & \\ & & 1\end{array}\right)$. 
VII. $-G_{5,},\left(x_{1}+x_{1}^{\prime}, e^{r x_{1}^{\prime}}\left(x_{2} \cos x_{1}^{\prime}-x_{3} \sin x_{1}^{\prime}\right)+x_{2}^{\prime}: e^{r x_{1}}\left(x_{2} \sin x_{1}^{\prime}+\right.\right.$ $\left.\left.+x_{3} \cos x_{1}{ }^{\prime}\right)+x_{3}{ }^{\prime}\right)$. Il est aisé de voir que les calculs sont les mêmes que pour $G_{3}$.

On arrive ainsi au résultat

$$
\begin{aligned}
\left(x_{1}, x_{2}, x_{3}\right) \rightarrow & \left(x_{1}, \alpha e^{r x_{1}} \cos x_{1}+\beta e^{r x_{1}} \sin x_{1}+\gamma x_{2}+\delta x_{3}-\alpha\right. \\
& \left.-\beta e^{r x_{1}} \cos x_{1}+\alpha e^{r x_{1}} \sin x_{1}-\delta x_{2}+\gamma x_{3}+\beta\right)
\end{aligned}
$$

les automorphismes intérieurs étant donnés par $\gamma=e^{r \varphi} \cos \varphi, \delta=e^{r \varphi} \sin \varphi$.

Les éléments de $\mathfrak{Q}\left(G_{5}, r\right)$ sont les matrices

$$
\left(\begin{array}{rrr}
\gamma & -\delta & \alpha \\
\delta & \gamma & \beta \\
0 & 0 & 1
\end{array}\right)
$$

et done il y a les représentations:

an) application constante

$$
\text { ao) } \xi_{x}=\left(\begin{array}{lll}
e^{x} & & \\
& e^{x} & \\
& & 1
\end{array}\right) \text {. }
$$

\section{§ 28. - Les groupes résolubles à 4 dimensions.}

Il est maintenant aisé d'écrire la loi de composition des extensions d'un groủpe à 3 dimensions par $R$, correspondant aux représentations données dans le paragraphe précédent.

I. - Extensions de $R^{3}$.

a) $R^{4}$

b) $R^{2} \times A$

c) $R \times G_{4}, r$

d) $\left(x_{1}+x_{1}^{\prime}, e^{x_{1}} x_{2}+x_{2}{ }^{\prime}, e^{r x_{1}{ }^{\prime}} x_{3}+x_{3}{ }^{\prime}, e^{s x_{1}^{\prime}} x_{4}+x_{4}^{\prime}\right)$

e) $R \times G_{3}$

f) $R \times G_{5}, r$

g) $\left(x_{1}+x_{1}^{\prime}, e^{r x_{1}}\left(x_{2} \cos x_{1}{ }^{\prime}-x_{3} \sin x_{1}\right)+x_{2}{ }^{\prime}, e^{r x_{1}{ }^{\prime}}\left(x_{2} \sin x_{1}{ }^{\prime}+x_{3} \cos x_{1}{ }^{\prime}\right)+\right.$ $\left.+x_{3}^{\prime}, e^{s x_{1}^{\prime}} x_{4}+x_{4}^{\prime}\right)$

h) $\left(x_{1}+x_{1}^{\prime}, x_{2} \cos x_{1}^{\prime}-x_{3} \sin x_{1}^{\prime}+x_{2}^{\prime}, x_{2} \sin x_{1}^{\prime}+x_{3} \cos x_{1}^{\prime}+x_{3}^{\prime}, e^{\left.r x_{1} x_{4}+x_{4}{ }^{\prime}\right)}\right.$

i) $R \times G_{1}$

j) $\left(x_{1}+x_{1}^{\prime}, x_{2}+x_{2}^{\prime}, x_{1}^{\prime} x_{2}+x_{3}+x_{3}^{\prime}, \frac{1}{2} x_{1}^{\prime 2} x_{2}+x_{1}^{\prime} x_{3}+x_{4}+x_{4}^{\prime}\right)$

k) $\left(x_{1}+x_{1}^{\prime}, x_{2}+x_{2}^{\prime}, e^{x_{1}^{\prime}} x_{3}+x_{3}^{\prime}, x_{1}^{\prime} x_{2}+x_{4}+x_{4}^{\prime}\right)$

l) $R \times G_{2}$

m) $\left(x_{1}+x_{1}^{\prime}, e^{x_{1}{ }^{\prime}} x_{2}+x_{2}{ }^{\prime}, e^{x_{1}{ }^{\prime}} x_{1}^{\prime} x_{2}+e^{x_{1}{ }^{\prime}} x_{3}+x_{3}^{\prime}, e^{r x_{1}{ }^{\prime}} x_{1}+x_{4}^{\prime}\right)$

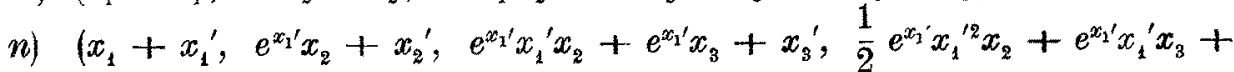
$\left.+e^{x_{1}^{\prime}} x_{4}+x_{4}^{\prime}\right)$. 
Parmi ceux de ces groupes qui sont des produits il n'y en a pas d'isomorphes; de plus aucun de ces groupes ne peut etre isomorphe à $d$ ), $g \mid, h$, $m$, n) car le sous-groupe des commutateurs de ces derniers est isomórphe a $R^{3}$. Pour la mème raison entre les groupes $d$ ), g), $h$ ), $m$ ), $n$ ) il $\mathbf{n}^{\prime} \mathrm{y}$ en a pas qui sont isomorphes, puisqu'il n'y en a pas qui le sont en tant qu'exten. sion. $j$ ) et $k$ ) ne sont pas isomorphes, $k$ ) contenant un sous-groupe isomorphe à $A$ et $j$ ) non. Pour les deux le centre est donné par $x_{1}=x_{8}=x_{3}=0$ : ce n'est pas un facteur direct, et done ni $j$ ) ni $k$ j ne peuvent être isomorphes à un produit.

II. - Extensions de $R \times A$.

o) $R^{2} \times A$

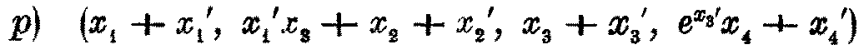

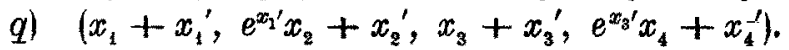

On voit que of est le groupe b); $p$ ) est nne extension de $R^{3} \cdot\left(x_{3}=0\right)$ et est done aussi contenu dans la liste précédente. q), qui est isomorphe à $A \times A$, ne figure pas encore, car il n'admet pas $R^{j}$ comme sous-groupe distingué.

III. - Extensions de $G_{1}$.

r) $R \times G_{1}$

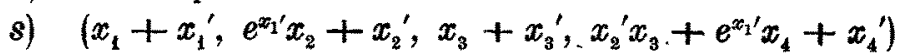

t) $\left(x_{1}+x_{1}^{\prime}, e^{x_{2}^{\prime}} x_{2}+x_{2}^{\prime}, e^{r x_{2}^{\prime}} x_{3}+x_{3}^{\prime}, e^{r x_{2}^{\prime}} x_{2}^{\prime} x_{3}+e^{(r+1) x_{1}^{\prime}} x_{4}+x_{4}^{\prime}\right)$

u) $\left(x_{1}+x_{1}^{\prime}, x_{2} \cos x_{1}^{\prime}-x_{3} \sin x_{1}^{\prime}+x_{2}^{\prime}, x_{2} \sin x_{1}^{\prime}+x_{3} \cos x_{1}^{\prime}+x_{3}^{\prime}\right.$, $\left.x_{2}^{\prime}\left(x_{2} \sin x_{1}^{\prime}+x_{3} \cos x_{1}^{\prime}\right)^{\prime}+\frac{1}{2}\left(x_{2}^{2}-x_{3}^{2}\right) \sin x_{1}^{\prime} \cos x_{1}^{\prime}-x_{2} x_{3} \sin ^{2} x_{1}^{\prime}+x_{4}+x_{4}^{\prime}\right)$

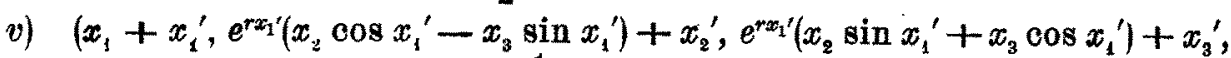
$x_{2}^{\prime} e^{r x_{1}^{\prime}}\left(x_{2} \sin x_{1}^{\prime}+x_{3} \cos x_{1}^{\prime}\right)+\frac{1}{2} e^{2 r x_{1}^{\prime}}\left(x_{2}^{2}-x_{3}^{2}\right) \sin x_{1}{ }^{\prime} \cos x_{1}^{\prime}-e^{2 r x_{1}^{\prime}} x_{2} x_{3} \sin ^{2} x_{1}^{\prime}+$ $\left.+e^{2 r x_{1}^{\prime}} x_{4}+x_{4}^{\prime}\right)$

w) $\left(x_{1}+x_{1}^{\prime}, x_{2}+x_{2}^{\prime}, x_{1}^{\prime} x_{2}+x_{2}+x_{3}^{\prime}, x_{2}^{\prime}\left(x_{1}^{\prime} x_{2}+x_{3}\right)+x_{4}+x_{4}^{\prime}\right)$

y) $\left(x_{1}+x_{1}^{\prime}, e^{x_{1}^{\prime}} x_{2}+x_{2}^{\prime}, e^{x_{1}^{\prime}} x_{1}^{\prime} x_{2}+e^{x_{1}^{\prime}} x_{3}+x_{3}^{\prime}, \cdot x_{2}^{\prime}\left(e^{x_{1}^{\prime}} x_{1}^{\prime} x_{2}+e^{x_{1}^{\prime}} x_{8}\right)+\right.$

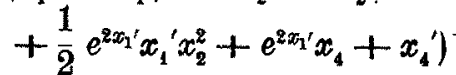

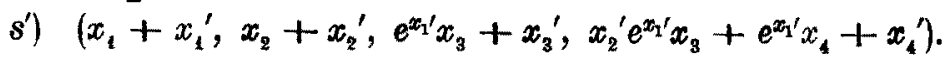

On a $r)=i$ ). Le sous-groupe $x_{2}=0$ de $w$ ) est isomorphe à $R^{3}$ et est distingué: $w$ ) figure donc parmi les groupes $a$ ) $-n$ ).

Les autres groupes de cette liste, n'admettant ni $R^{3}$ ni $A$ comme sousgroupe distingué, ne sont pas compris dans les listes précédentes, $s$ ) et $s^{\prime}$ ) sont les senls dont le sous-groupe des commutateurs est $R^{2}$ : mais $s^{\prime}$ ) admet $G_{2}$ comme sous-groupe distingué $\left(x_{1}=x_{2}\right)$ ce qui n'est pas le cas pour $\left.s\right)$. $\left.t\right)$ n'est pas isomorphe à $y$ ), ce dernier étant une extension de $R$ par $G_{2}$ : les deux, ils sont differents de $u$ ) et de $v$ ) qui, la valeur $r=0$ etant exclue, ne sont évidemment pas isomorphes. 
IV. - Extensions de $G_{2}$.

aa) $R \times G_{2}$

ab) $\left(x_{1}+x_{1}^{\prime}, x_{2}+x_{2}^{\prime}, e^{x_{2}^{\prime}} x_{3}+x_{3}^{\prime}, e^{x_{2}^{\prime}} x_{2}^{\prime} x_{3}+x_{1}^{\prime} x_{3}+e^{x_{2}^{\prime}} x_{4}+x_{4}{ }^{\prime}\right)$

$a a)=l$ ). En posant $x_{2}=0$, on voit que $a b$ ) est une extension de $G_{1}$ et done qu'il est dójà parmi les groupes considérés plus haut.

V. - Extensions de $G_{3}$.

ac) $R \times G_{3}$

ad) $\left(x_{1}+x_{1}^{\prime}, x_{2}+x_{2}^{\prime}, e^{v_{1}^{\prime}}\left(x_{3} \cos x_{2}{ }^{\prime}-x_{4} \sin x_{2}{ }^{\prime}\right)+x_{3}^{\prime}, e^{x_{1}^{\prime}}\left(x_{3} \sin x_{2}{ }^{\prime}+\right.\right.$ $\left.\left.+x_{4} \cos x_{2}^{\prime}\right)+x_{4}^{\prime}\right)$

$a c)=e$ ). $a d$ ) n'est isomorphe à aucun autre des groupes considérés car il n'est extension ni de $R^{3}$ ni de $A$ ni de $G_{1}$ ou $G_{2}$.

VI. - Extensions de $G_{4,1}$.

ae) $R \times G_{4,1}$

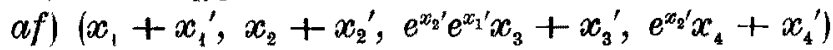

ag) $\left(x_{1}+x_{1}^{\prime}, x_{2}+x_{2}^{\prime}, e^{x_{2}^{\prime}} x_{3}+x_{3}^{\prime}, x_{1}^{\prime} x_{3}+e^{x_{2} x_{4}}+x_{4}^{\prime}\right)$

ah) $\left(x_{1}+x_{1}^{\prime}, x_{2}+x_{2}^{\prime}, e^{x_{2}} e^{x_{1}^{\prime}} x_{3}+x_{3}^{\prime}, x_{1}^{\prime} e^{x_{1}^{\prime}} x_{3}+e^{x_{2}{ }^{\prime}} e^{x_{1}{ }^{\prime}} x_{4}+x_{4}^{\prime}\right)$

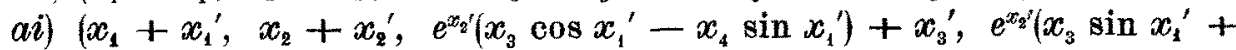
$\left.\left.+x_{4} \cos x_{1}^{\prime}\right)+x_{4}^{\prime}\right)$

aj) $\left(x_{1}+x_{1}^{\prime}, x_{2}+x_{2}^{\prime}, e^{x_{2}^{\prime}} e^{x_{1}^{\prime}} x_{3}+x_{3}^{\prime}, e^{\left.x_{2} e^{\prime r x_{1}} x_{4}+x_{4}{ }^{\prime}\right)}\right.$

ak) $\left(x_{1}+x_{1}^{\prime}, x_{2}+x_{2}^{\prime}, e^{x_{1}^{\prime}} e^{r x_{1}^{\prime}}\left(x_{3} \cos x_{1}^{\prime}-x_{4} \sin x_{1}^{\prime}\right)+x_{3}, e^{x_{2}^{\prime}} e^{r x_{1}^{\prime}}\left(x_{3} \sin x_{1}^{\prime}+\right.\right.$ $\left.\left.+x_{4} \cos x_{1}^{\prime}\right)+x_{4}^{\prime}\right)$.

$a e)=c)$. Le sous-groupe $x_{2}=0$ de $a f$ ) est isomorphe à $R \times A$ et est distingué; ce groupe figure done dejà dans les listes précédentes. De même ag) qui est une extension de $G_{1}\left(x_{2}=0\right)$, ah) qui est une extension de $G_{2}\left(x_{1}=x_{2}\right)$. $\left.\left.a i\right)=a d\right)$, après permutation de $x_{1}$ et $x_{2}$. aj) devient, pour $r \neq 1, A \times A$ en posant $\bar{x}_{1}=x_{1}+x_{2}, \quad \bar{x}_{2}=r x_{1}+x_{2} ;$ et devient $R \times G_{4, s}$ si $r=1$ en posant $\vec{x}_{1}=x_{1}+x_{2}$. Enfin ak) est une extension de $G_{3}\left(x_{2}=r x_{1}\right)$.

Extensions de $G_{4}, ., r \neq 1$.

al) $R \times G_{4}, r$

am) $\left(x_{1}+x_{1}^{\prime}, x_{2}+x_{2}{ }^{\prime}, e^{x_{2}{ }^{\prime}} x_{3}+x_{3}{ }^{\prime}, e^{r x_{2}^{\prime}} e^{x_{1}^{\prime}} x_{4}+x_{4}{ }^{\prime}\right)$.

$a l)=c$ ). am) n'est autre que $A \times A$ en posant $\bar{x}_{1}=x_{1}+r x_{2}$.

VII. Extensions de $G_{5}, \ldots$

an) $R \times G_{5}$,

ao) $\left(x_{1}+x_{1}^{\prime}, x_{2}+x_{2}^{\prime}, e^{x_{1}^{\prime}}\left(x_{3} \cos x_{2}^{\prime}-x_{4} \sin x_{2}^{\prime}\right)+x_{3}^{\prime}, e^{x_{1}^{\prime}}\left(x_{3} \sin x_{2}^{\prime}+\right.\right.$ $\left.+x_{1} \cos x_{2}^{\prime}\right)+x_{1}^{\prime}$.

$a n=f$ ) et $a o)=a d$ ). 
Résumant, et omettant les produits de deux groupes de dimensions inférieures, voici la liste des groupes de LiE résolubles simplement connexes à 4 dimensions.

- Groupes admettant $R^{3}$ comme sous-groupe distingué $\left({ }^{37}\right)$.

1) $\left(x_{1}+x_{1}^{\prime}, x_{2}+x_{2}^{\prime}, e^{x_{1}^{\prime}} x_{3}+x_{3}^{\prime}, x_{1}^{\prime} x_{2}+x_{4}+x_{4}^{\prime}\right) . \quad c_{133}=1, \quad c_{124}=1$, centre $x_{1}=x_{2}=x_{3}=0, E\left(R, R^{3}\right), E\left(R^{2}, R^{2}\right), E\left(G_{1}, R\right)$.

2) $\left(x_{1}+x_{1}^{\prime}, x_{2}+x_{2}^{\prime}, x_{1}^{\prime} x_{2}+x_{3}+x_{3}^{\prime}, \frac{1}{2} x_{1}^{\prime 2} x_{2}+x_{1}^{\prime} x_{3}+x_{4}+x_{4}^{\prime}\right), c_{123}=1$, $c_{134}=1$, contre $x_{1}=x_{2}=x_{3}=0, E\left(R, R^{3}\right), E\left(R, G_{1}\right), E\left(R^{2}, R^{2}\right), E\left(G_{1}, R\right)$.

3) $\left(x_{1}+x_{1}^{\prime}, e^{x_{1}^{\prime}} x_{2}+x_{2}^{\prime}, e^{x_{1}^{\prime}} x_{1}^{\prime} x_{2}+e^{x_{1}^{\prime}} x_{3}+x_{3}^{\prime}, \frac{1}{2} e^{x_{1}^{\prime}} x_{1}^{\prime 2} x_{2}+e^{x_{1}^{\prime}} x_{1}^{\prime} x_{3}+\right.$

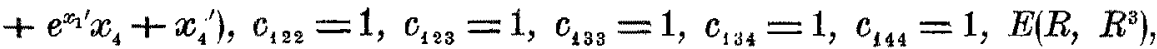
$E\left(A, R^{2}\right), E\left(G_{2}, R\right)$.

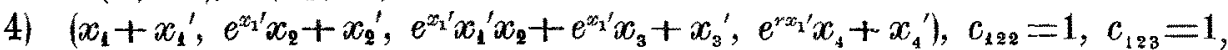
$c_{133}=1, \quad c_{144}=r, \quad r \neq 0, E\left(R, R^{3}\right), E\left(A, R^{2}\right), E\left(G_{2}, R\right), E\left(G_{4}, r, R\right)$.

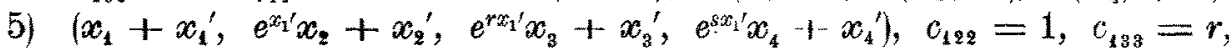
$r \neq 0, c_{144}=s, s \neq 0, E\left(R, R^{3}\right), E\left(A, R^{2}\right), E\left(G_{4}, \ldots, R\right), E\left(G_{4}, s, R\right)$.

6) $\left(x_{1}+x_{1}^{\prime}, e^{r x_{1}} x_{2}+x_{2}^{\prime}, x_{3} \cos x_{1}^{\prime}-x_{4} \sin x_{1}^{\prime}+x_{3}^{\prime}, x_{3} \sin x_{1}^{\prime}+x_{4} \cos x_{1}^{\prime}+\right.$, $\left.+x_{4}^{\prime}\right), c_{122}=r, r \neq 0, c_{143}=-1, c_{134}=1, E\left(R, R^{3}\right), E\left(G_{3}, R\right)$.

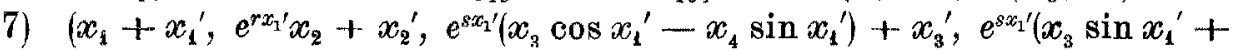
$\left.\left.+x_{4} \cos x_{1}^{\prime}\right)+x_{4}^{\prime}\right), \quad c_{122}=r, \quad r \neq 0, \quad c_{133}=s, \quad c_{143}=-1, \quad c_{134}=1$, $c_{144}=s, s>0, E\left(R, R^{3}\right), E\left(G_{5}, s, R\right)$.

- Groupes $n^{\prime}$ admettant pas $R^{3}$ comme sous-groupe distingué.

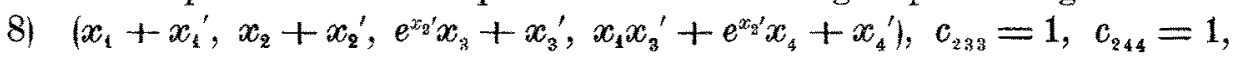
$c_{134}=1, E\left(R, G_{1}\right), E\left(R, G_{4,1}\right), E\left(R^{2}, R^{2}\right), E\left(A, R^{2}\right), E(A \times R, R)$.

9) $\left(x_{1}+x_{1}{ }^{\prime}, e^{x_{1}{ }^{\prime}} x_{2}+x_{2}{ }^{\prime}, e^{r x_{1}{ }^{\prime}} x_{3}+x_{3}{ }^{\prime}, e^{r x_{1}} x_{2}{ }^{\prime} x_{3}+e^{(r+1) x_{1}{ }^{\prime}} x_{4}+x_{4}{ }^{\prime}\right), c_{122}=1$, $c_{133}=r, c_{144}=r+1, r \neq 0, c_{234}=1, E\left(R, G_{1}\right), E\left(A, R^{2}\right), E\left(G_{4}, n, R\right)$.

10) $\quad\left(x_{1}+x_{1}^{\prime}, e^{x_{1}{ }^{\prime}} x_{2}+x_{2}^{\prime}, e^{x_{1}{ }^{\prime}} x_{1}^{\prime} x_{2}+e^{x_{1}} x_{3}+x_{3}^{\prime}, e^{x_{1}{ }^{\prime}} x_{1}^{\prime} x_{2}{ }^{\prime} x_{3}+\frac{1}{2} e^{2 x_{1}{ }^{\prime}} x_{1}^{\prime} x_{2}^{2}+\right.$ $\left.+e^{x_{1}^{\prime}} x_{2}^{\prime} x_{3}+e^{2 x_{1}^{\prime}} x_{4}+x_{4}^{\prime}\right), c_{122}=1, c_{123}=1, c_{133}=1, c_{144}=2, c_{234}=1$, $E\left(R, G_{1}\right), E\left(A, R^{\prime}\right), E\left(G_{2}, R\right)$.

11) $\left(x_{1}+x_{1}^{\prime}, x_{2} \cos x_{1}^{\prime}-x_{3} \sin x_{1}^{\prime}+x_{2}^{\prime}, x_{2} \sin x_{1}^{\prime}+x_{3} \cos x_{1}^{\prime}+x_{3^{\prime}}\right.$ $x_{2}^{\prime}\left(x_{2} \sin x_{1}^{\prime}+x_{3} \cos x_{1}^{\prime}\right)+\frac{1}{2}\left(x_{2}^{2}-x_{3}^{2}\right) \sin x_{1}^{\prime} \cos x_{1}^{\prime}-x_{2} x_{3} \sin ^{2} x_{1}{ }^{\prime}+$ $\left.+x_{4}+x_{4}^{\prime}\right), c_{432}=-1, c_{123}=1, c_{234}=1$, centre $x_{1}=2 k-x_{2}=x_{3}=$ $=x_{4}=0, E\left(R, G_{1}\right), E\left(G_{3}, R\right)$.

12) $\quad\left(x_{1}+x_{1}^{\prime}, e^{r x_{1}}\left(x_{2} \cos x_{1}^{\prime}-x_{3} \sin x_{1}^{\prime}\right)+x_{2}^{\prime}, e^{r x_{1}}\left(x_{2} \sin x_{1}^{\prime}+x_{3} \cos x_{1}^{\prime}\right)+\right.$ $+x_{3}^{\prime}, e^{r x_{1}^{\prime}} x_{2}^{\prime}\left(x_{2} \sin x_{1}^{\prime}+x_{3} \cos x_{1}^{\prime}\right)+\frac{1}{2} e^{2 r x_{1}^{\prime}}\left(x_{2}^{2}-x_{3}^{2}\right) \sin x_{1}^{\prime} \cos x_{1}^{\prime}-$

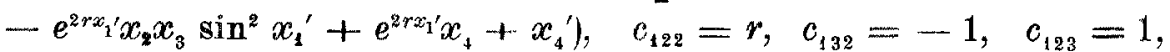
$c_{133}=r, \quad c_{444}=2 r, \quad r>0, \quad c_{234}=1, E\left(R, G_{1}\right), E\left(G_{5}, \ldots, R\right)$.

(37) Le groupe 1) (resp. 5)) de la liste des groupes résolubles de [6] est isomorphe ì $R \times G_{1}($ resp. $\because 6)$ aveo $\left.r=1\right)$. 
13) $\left(x_{1}+x_{1}^{\prime}, x_{z}+x_{2}^{\prime}, e^{x_{2}{ }^{\prime}} x_{1}^{\prime} x_{4}+e^{x_{2}} x_{3}+x_{3}^{\prime}, e^{x_{2}{ }^{\prime}} x_{4}+x_{4}^{\prime}\right), c_{283}=1, c_{143}=1$, $c_{244}=1, E\left(R, G_{1}\right), E\left(R, G_{2}\right), E\left(R, G_{4}, 1\right), E\left(R^{2}, R^{2}\right), E\left(A, R^{2}\right), E(R \times A, R)$.

14) $\left(x_{1}+x_{1}^{\prime}, x_{2}+x_{2}^{\prime}, e^{x_{2}^{\prime}}\left(x_{3} \cos x_{1}^{\prime}-x_{4} \sin x_{1}^{\prime}\right)+x_{3}^{\prime}, e^{x_{2}^{\prime}}\left(x_{3} \sin x_{1}^{\prime}+\right.\right.$ $\left.\left.+x_{4} \cos x_{1}^{\prime}\right)+x_{4}^{\prime}\right), \quad c_{233}=1, \quad c_{143}=-1, \quad c_{244}=1, \quad c_{134}=1$, centre $x_{1}=2 k \pi, x_{2}=x_{3}=x_{4}=0, E\left(R, G_{3}\right), E\left(R, G_{4}, 1\right), E\left(R, G_{5}, r\right), E\left(R^{2}, R\right)$.

\section{Chapitre V. - LES eXTeNSTONS DES Algebres DE LIE}

\section{§ 29. - Généralités.}

Les définitions concernant la théorie des extensions des algèbres de LIE peuvent s'obtenir des définitions données dans les chapitres précédents par des modifications formelles evidentes. Nous noterons $\mathbf{e}(\mathbf{b}, \mathbf{f})$ une algèbre de LIE extension de f par b, par $x$ (resp. $y$ ) les éléments de b (resp. de f). Au lieu du groupe $\mathfrak{A}\left(F^{\prime}\right)$ (resp. $\mathfrak{d}(F)$ ) on considérera maintenant l'algèbre a(f) (resp. i(f)) des dérivations (resp. des dérivations intérieures) de f.

Une préreprésentation de $\mathbf{b}$ dans a(f) est une application linéaire $x \rightarrow \xi_{x}$ telle que

$$
\xi_{\left[x_{1}, x_{2}\right]}=\xi_{x_{1}} \xi_{x_{2}}-\xi_{x_{2}} \xi_{x_{1}}+a d h\left(x_{1}, x_{2}\right)
$$

$h$ étant une application de $\mathbf{b} \times \mathbf{b}$ dans $\mathbf{f}$. Par projection $\xi$ donne une représentation de b dans $\varepsilon(\mathbf{f})=\mathbf{a}(\mathbf{f}) / \mathbf{i}(\mathbf{f})$.

Une $n$-cochaîne $g(n>0)$ de b à valeurs dans $f$ est une application linéaire altérnée de $\mathbf{b} \times \mathbf{b} \times \ldots \times \mathbf{b}(n$ facteurs $)$ dans $\mathbf{f}$; une 0 -cochaîne est un elément de $\mathbf{f}$.

Le cobord $\delta g$ de $g$ relatif à $\xi$ est défini par

$$
\begin{aligned}
(\delta g)\left(x_{1}, x_{2}, \ldots, x_{n+1}\right) & =\sum_{i=1}^{n+1}(-1)^{i+1} \xi_{r_{i}}\left\{g\left(x_{1}, x_{2}, \ldots, \widehat{x_{i}}, \ldots, x_{n+1}\right)\right\}+ \\
& +\sum_{i<j}^{\sum}(-1)^{i+j} g\left(\left[x_{i}, x_{j}\right], x_{1}, \ldots, \widehat{x_{i}}, \ldots, \widehat{x_{j}}, \ldots, x_{n+1}\right)
\end{aligned}
$$

le symbole $\widehat{x_{i}}$ désignant que l'argument $x_{i}$ est à omettre. Le cobord d'une 0 -cochaine $y$ est donné par $(\delta y)(x)=-\xi_{x}(y)$.

Un cocycle relatif à $\xi$ est une cocialne dont le cobord relatif à $\xi$ est nul. On voit immédiatement que les $n$-cocycles (resp. les $n$-cobords) relatifs à $\xi$ forment un groupe abélien $Z^{n}(\mathbf{b}, \mathbf{f}, \xi)$ (resp. $B^{n}(\mathbf{b}, \mathbf{f}, \xi)$ ). Si $\xi$ est une représentation, on vérifie que $\delta \delta g=0$ pour toute cochaîne $g$ : on peut alors définir le $n$-ième groupe de cohomologie de $\mathbf{b}$ à valeurs dans $\mathbf{f}$ relatif à $\xi$ : $H^{n}(\mathbf{b}, \mathbf{f}, \xi)=Z^{n}\left(\mathbf{b}, \mathbf{f}, \xi_{1} / B^{n}(\mathbf{b}, \mathbf{f}, \xi)\left({ }^{38}\right)\right.$.

(38) Pour ces définitions ofr. [11], \$ 23. 
Comme pour les groupes, nous noterons par $(g, \xi)$ les 2-cocycles relatifs à $\xi$ tels que

$$
\xi_{\left[x_{1}, x_{2}\right]}=\xi_{x_{1}} \xi_{x_{2}}-\xi_{x_{2}} \xi_{x_{1}}-a d g\left(x_{1}, x_{2}\right) \text {. }
$$

Deux cocycles $(g, \xi)$ et $(f$, I) de $\mathbf{b}$ à valeurs dans $\mathbf{f}$ sont dits équivalents s'il existe une 1-cochaîne $h$ telle que

$$
\begin{gathered}
f\left(x_{1}, x_{2}\right)=g\left(x_{1}, x_{2}\right)-\xi_{x_{2}}\left\{h\left(x_{1}\right)\right\}+\xi_{x_{1}}\left\{h\left(x_{2}\right)\right\}-h\left(\left[x_{1}, x_{2}\right]\right)+\left[h\left(x_{1}\right), h\left(x_{2}\right)\right] \\
\vartheta_{x}=\xi_{x}+a d h(x) .
\end{gathered}
$$

On constate alors facilement $q u$ ' une classe d'équivalence de cocycles $(g, \xi)$ : réciproquement un tel cocycle definit sur l'espace vectoriel somme $b+f$ une structure d'extension avec la loi de composition

$$
\left[x_{1}+y_{1}, x_{2}+y_{2}\right]=\left[x_{1}, x_{2}\right]+g\left(x_{1}, x_{2}\right)+\xi_{x_{1}}\left(y_{2}\right)-\xi_{x_{2}}\left(y_{1}\right)+\left[y_{1}, y_{2}\right]:
$$

à deux cocycles équivalents correspondent alors deux extensions équivalentes.

Si $q$ dẻsigne la projection canonique de a(f) sur $\boldsymbol{\varepsilon}(\mathbf{f})$ et $q$ une prérepré. sentation de b dans a(f), tout cocycle $(g, \xi)$ de $\mathbf{b}$ à valeurs dans $\mathbf{f}$ tel que $q \xi=q^{\vartheta}$ est équivalent à un cocycle $\left(g^{\prime}\right.$, \$). (29.2) montre alors que si $\left(f, \vartheta^{\prime}\right)$ est un autre cocycle, $\left(f-g^{\prime}\right.$, श) est un cocycle de $\mathbf{b}$ à valeurs dans le cen. tre e de f. On en déduit:

Proposimion 29.1. - $\mathbf{b}$, $\mathbf{f}$ et la représentation $\chi$ de $\mathbf{b}$ dans $\varepsilon(\mathbf{f})$ étant donnés, s'il $y$ a des cocycles $(\mathrm{g}, \xi)$ de $\mathbf{b}$ à valeurs dans $\mathbf{f}$ tels que $\mathrm{q} \xi=\chi$, l'ensemble de leurs classes d'équivalence est en correspondance birnivoque avec $\mathrm{H}^{2}(\mathbf{b}, \mathbf{c}, \chi)$ (cfr. Prop. 2.4).

Nous désignons par la lettre $\chi$ aussi la représentation de b dans a(c) $q u^{\prime}$ on déduit de $\xi$ et qui ne dépend que de $q \xi$.

COROLlaIRE 1. - S'il y a des extensions $\mathbf{e}(\mathbf{b}, \mathbf{f})$ de caractère $\chi$, l'ensemble de leurs classes d'équivalence est en correspondance biunivoque avec $\mathrm{H}^{2}(\mathbf{b}, \mathbf{c}, \xi)$.

CoRolulatre 2. - S'il $y$ a des extensions préinessentielles $\mathbf{e}(\mathbf{b}, \mathbf{f})$ de caractère $\chi$, toutes les extensions de $\mathbf{f}$ par $\mathbf{b}$ de même caractère sont préinessentielles et l'ensemble de leurs classes d'équivalence est un groupe isomorphe $\dot{a} \mathbf{H}^{2}(\mathbf{b}, \mathbf{c}, \chi)$ (cfr. Prop. 9.2, Coroll. 5).

\section{30. - Extensions d'algèbres abéliennes.}

Puisque toute $n$-cochẫne est une application multilineaire alternée, si b est nne algèbre de LIE à une dimension toute $n$-coehaîne avec $n>1$ est constante :

Proposition 30.1. - Toute extension $\mathrm{e}(\mathrm{b}, \mathbf{f})$ où $\mathbf{b}$ est une algèbre de Lie à une dimension est inessentielle (cfr. Prop. 22.1, Coroll. 1). 
Supposons $b$ et $\mathbf{f}$ abéliennes et cherchons les extensions abéliennes : par définition le 2-cocycle $g$ est donné par $\left[u\left(x_{1}\right), u\left(x_{2}\right)\right]=u\left(\left[x_{1}, x_{2}\right]\right)+g\left(x_{1}, x\right)$, $u$ étant un système de représentants de $b$ dans $\rho:$ on voit que $\boldsymbol{e}$ abélienne implique $g=0$. Comme de plus $\mathbf{e}(\mathbf{b}, \mathbf{f})$ doit être centrale on a:

Proposition 30.2. - b et f étant deux algèbres de Lie abéliennes, toute extension $\mathrm{e}(\mathbf{b}, \mathbf{f})$ abélienne est triviale (cfr. Prop. 18.5).

Si $f$ a la dimension 1 , nous pouvons ecrire $\xi_{x}(y)=\lambda_{x} y$. $\lambda_{x}$ étant un nombre réel; pour b abélienne (29.1) devient

$$
\lambda_{x_{1}} g\left(x_{2}, x_{3}\right)-\lambda_{x_{2}} g\left(x_{1}, x_{3}\right)+\lambda_{x_{3}} g\left(x_{1}, x_{2}\right)=0 ;
$$

résolvant par rapport à $g\left(x_{1}, x_{2}\right)$, on voit que $g$ est le cobord de $h(x)=$ $=g\left(x, x_{3}\right) / \lambda_{x_{3}}$.

Lemme 30.1. - Soient $\mathbf{b}$ et $\mathbf{f}$ deux algèbres de Lie dont $\mathbf{b}$ abélienne et $\mathbf{f} \grave{\alpha}$ une dimension. Toute extension $\mathbf{e}(\mathbf{b}, \mathbf{f})$ est centrale ou inessentielle (cfr. Prop. 19.1).

Puisqu' une cochaine est une application multilinéaire, si $x_{1}, x_{2}, \ldots, x_{m}$ est une base de $b$ on a toujours

$$
g\left(\sum_{i=1}^{m} \lambda_{i} x_{i}, \sum_{i=1}^{m} \mu_{i} x_{i}\right)=\sum_{i<j}\left(\lambda_{i} \mu_{j}-\lambda_{j} \mu_{i}\right) g\left(x_{i}, x_{j}\right) ;
$$

$y_{1}, y_{2}, \ldots, y_{n}$ étant ùne base de $\mathbf{f}$, posons $g\left(x_{i}, x_{j}\right)=\sum_{k=1}^{n} p_{i j k} y_{k}:$

$$
g\left(\sum_{i=1}^{m} \lambda_{i} x_{i}, \sum_{i=1}^{m} \mu_{i} x_{i}\right)=\sum_{\substack{k=1 \\ i<j}}^{n}\left(\lambda_{i} \mu_{j}-\lambda_{j} \mu_{i}\right) \rho_{i j k} y_{k} .
$$

Puisque toute cochaine d'une algèbre de Lre abélienne est un cocycle relatif à $\xi=$ const., (30.1) donne le cocycle associé à une extension centrale:

Proposition 30.3. - b étant une algèbre de Lie abélienne, à toute exten. sion centrale $\mathrm{e}(\mathrm{b}, \mathbf{f})$ est associé un cocycle $(\mathrm{g}, \xi)$ où $\xi=$ const. et $\mathrm{g}$ est donné par la formule (30.1) et prend ses valeurs dans le centre de $\mathbf{f}$.

\section{§ 31. - Les algèbres semi-simples.}

Puisque toute dérivation d' une algèbre semi-simple est intérieure, toute extension d'une telle algèbre est centrale: mais comme elle n'a pas de cen. tre on obtient:

Proposition 31.1. - Soit f une algèbre de Lie semi-simple: toute extension $\mathbf{e}(\mathbf{b}, \mathbf{f})$ est équivalente à l'extension triviale (cfr. remarque b) à la fin du \& 15).

On peut en conclure:

CoRollaire. - Une algèbre de Lie b étant donnée, pour que toute extension $\mathbf{e}(\mathbf{b}, \mathbf{f})$ soit inessentielle, il faut et il suffit que toute extension $\mathbf{e}^{\prime}\left(\mathbf{b}, \mathbf{f}^{\prime}\right)$ avec $\mathbf{f}^{\prime}$ abélien soit inessentielle (cfr. Prop. 22.1 et [11], thé. 26.1). 
Chevallex et Eilenberg ont démontré la

Proposimion 31.2. - ([11], thé. 21.1 et thé. 24.1). Soit $\mathbf{b}$ une algèbre de Lie semi-simple. Pour tout algèbre de Lie $\mathbf{f}$ abélienne et pour toute représentation $\xi$ de $\mathbf{b}$ dans $\mathbf{a}(\mathbf{f})$, le groupe $\mathrm{H}^{2}(\mathbf{b}, \mathbf{f}, \xi)$ est réduit à l'élément neutre (cfr. Coroll. 2 à la Prop. 23.1).

Corollatre 1. - (Théorème de Levi). Toute extension $\mathbf{e}(\mathbf{b}, \mathbf{f})$ avec $\mathbf{b}$ semisimple est inessentielle.

Comme pour les groupes, de ce theorème suit le

CoRollaire 2. - b étant une algèbre de Lie semi-simple, pour toute représentation $\chi$ de $\mathbf{b}$ dans $\varepsilon(\mathbf{f})$ il existe une extension de l'algèbre $\mathbf{f}$ par $\mathbf{b}$ de caractère $\chi$ (cfr. Prop. 23.2).

On peut obtenir un critère d'existence général par les considérations suivantes. Soit $\chi$ une représentation non constante de $b$ dans $\varepsilon(\mathbf{f}), u$ un systeme de représentants de $\varepsilon(\mathbf{f})$ dans a(f) qui définit le cocycle $(\bar{g}, \bar{g})$; le cocycle transposé $\chi^{*}(\bar{g}, \bar{\xi})$ est un cocycle de $\mathbf{b}$ à valeurs dans $\mathbf{f} / \mathbf{e}$, que nous identifions à $\mathbf{i}(\mathbf{f})$. Soit $v$ un système de représentants de $\mathbf{f} / \mathrm{c}$ dans $\mathbf{f}$ et $g=v \chi^{*} \bar{g}$. Il est clair que $\delta g=h$ (le cobord étant pris par rapport à $\xi_{x}=u\left(\chi_{x}\right)$ ) est un élément de $Z^{3}(\mathbf{b}, \mathbf{c}, \chi)$. On vérifie alors que la classe de cohomologie de $h$ est indépendante du choix de $u$ et de $v$. De plus on peut faire parcourir à $h$ toute sa classe de cohomologie. Ainsi si $h$ est un cobord, nous ponvons sup. poser $h=0$, et done $\delta g=0 .(29.2)$ étant satisfait, $(g, \xi)$ est un cocycle associé à une extension $\mathrm{e}(\mathbf{b}, \mathbf{f})$ de caractère $x$.

Si nous appelons classe carastéristique de $(b, f, \chi)$ la classe de cohomo. logie de $h$ et, pour $\chi=$ const., la classe de cohomologie zéró, on peut formuler:

Proposimion 31.3. - Pour qui il existe une extension $\mathbf{e}(\mathbf{b}, \mathbf{f})$ de caractère $\chi$, il faut et il suffit que la classe caractéristique de $(\mathbf{b}, \mathbf{f}, \chi)$ soit nulle (cfr. [18], thé. 8.1).

\section{BIBLIOGRAPHIE}

[1] R. BAER, Erveiterung von Gruppen und ihren Isomorphismen "Math. Zeit. ", 38, (1934), pag. $375 \cdot 416$.

[2] A. Boner, Sections locales de certains espaces fibrés, "C. R. Acad. Sci. Paris", 230, (1950), pag. 1246.1248.

[3] N. Bourbakr, Algèbre, chap. I, Hermann, Paris, (1942)

[4] - Topologie générale, Hermann, Paris, (1940-49).

[ö] J. Braconniar, Suv les groupes topologiques localement compacts, "Jour. Math. ", 27, (1948), pag. 1.85.

[6] L. Calabi, Les groupes de Lie réels à 4 dimensions, "Bull. Acad. Roy. Belgique», 36, (1950). pag. 579.086 .

[7] - - Le estensioni centrali di gruppi, * Boll Un. Mat. Italiana", 5, (1950), pag. 264.266, Su alcuni rapporti tra la teoria delle estensioni ed il gruppo degli automorfismi del gruppo esteso, ibidem, pag. 286.289.

[8] E. CaRtaN, Les groupes réels simples, finis et continus; "Ann. E. N. S. Paris", 31, (1914), pag. $26 . .3555$. 
[8'] - La théorie des groupes finis et continus et l'analysis situs, Mémorial 42, Paris, 1930.

[9] C. Chevaltey, Two theorems on solvable topological groups, Lectures in topology, University of Michigan Press, (1941), pag: 291.92.

[10] - - Theory of Lie groups, Princeton University Press, (1946).

[11] O. Chevalley-S. Erlenberg, Cohomology theory of Lie groups and Lie algebras, "Trans. Nat. Acad. Sci. U.S.A. s, 63, (1948), pag. 85.124.

[12] A. H. ClIFFORD-S. MACLANe, Factor-sets of a growp in its abstract unit group, "Trans. Am. Math. Soc.", 50, (1941), pag. 385-406.

[13] C. EHRESMANN, Sur les applications continues d'un espace dans un espace fibré ou dans un revêtement, \& Bull. Soc. Math. France», 72, (1944), pag. 27.54.

[14] - - Sur les sections d'un champ d'élements de contact dans une variété différentiable, « C. R. Acad. Sci. Paris ₹, 224, (1947), pag. 444-445.

[15] - Les connexions infinitésimales dans un espace fibré différentiable, Colloque de Topologie, Bruxelles, Juin 1950.

[16] S. Embnberg-S. MacLane, Group extensions and homology, "Ann. of Math. ", 43 , (1942), pag. $757-831$.

[17] - Cohomology theory in abstract groups I, "Ann. of. Math. ", 48, (1947], pag. 51.78.

[18] - Cohomology theory in abstract groups II, "Ann. of Math. ", 48, (1947), pag. 326-341.

[19] A. M. Glaseson, On the structure of locally compact groups, "Proc. Math. Acad. SciU.S.A. ", 35, (1949), pag. 384-386.

[20] J. A. Golfand, Sur les isomorphismes des extensions de groupes, "Doklady Acad. Sei. URSS », 60, (1948).

[21] K. IWASAWA, On some types of topological groups, *Ann. of Math. „, 50, (1949), pag. 507.558.

[22] B. De KerekJarto, Sur les groupes intégrables d'ordre 3, "Math. Rew* ", 9, (1948), pag. 330.

[23] E. R. Kolohin, On certain concepts in the theory of algebraic matric groups, Ann. of Math. », 49, (1948), pag. 774-789.

[24] H. C. Les, Sur les groupes de Lie réels à 3 paramètres, "Jour. Math. „, 26, (1947): pag. 251-267.

[25] $\Delta$. MALCw, On the representation of an alyebra as a direct sum of the radical and a semi-simple subalgebra, "Doklady Acad. Sc. URSS ", 36, (1942), pag. 42-4j.

[26] H. NAGAO, The extensions of topological groups, “ Osaka Math. Jour. ", 1, (1949), pag. 36.42.

[27] L. Pontriagin, Topological groups, Princeton University Press, (1946).

[28] O. SchrnIER, Ueber die Erweiterung von Gruppen I, Monatshefte Matb. Phys. ", 34, (1926), pag. 164-180; Ueber die Erweiterung von Gruppen $I I$, Hamburg Abh. * 4, (1926), pag. 321-346.

[29] J. P. Serre, Compacité locale des espaces fibrés, a C. R. Acad. Sci. Paris s, 229, (1949), pag. $1295 \cdot 1297$.

[30] - - Trivialité des espaces fibrés, «C. R. Acad. Sci. Paris „, 230, (1950), pag. 916.918.

[31] A. Shapiro, Group extensions of compact Lie groups, * Ann. of Math. *, 50, (1949), pag. 581586 .

[32] A. SpeIser, Zahlentheoretische Sätze aus der Gruppentheorie, * Math. Zeit. ", 5, (1919), pag. 1.6.

[39] - - Theorie der Gruppen von endlicher oranung, Springer, Berlin, (1987).

[34] A. M. TuRing, The extensions of a group, "Compositio Math. ", 5, (1938), pag. 357-367.

[30] X. J. VILENkiN, Sur les groupes faiblement séparables, "Math. Sbornik ", 22, (1948), pag. 135-177.

[36] A. WEIL, L'intégration dans les groupes topologiques, Hermann, Paris, 1940.

[37] H. ZassenhaUs, Lehrbuch der Gruppentheorie, Teubner, Leipzig, 1937. 